\title{
Catalytic, Regioselective Hydrocarbofunctionalization of Unactivated Alkenes Triggered by trans-Acetoxypalladation of
}

\author{
Alkynes \\ Rahul K. Shukla, Atul K. Chaturvedi, Subir Pal, Chandra M. R. Volla* \\ Department of Chemistry, Indian Institute of Technology Bombay \\ Powai, Mumbai-400076, India. \\ E-mail: chandra.volla@chem.iitb.ac.in
}

\section{Supporting Information}

Table of Contents

1) General considerations and reagent information $\quad$ S2

2) General procedure for the synthesis of starting materials $\quad$ S2

3) Optimization details $\quad$ S4

4) General synthetic procedures $\quad$ S4

5) Proposed reaction mechanism $\quad$ S5

6) Characterization data S6

7) Scale up and Functionalization $\quad$ S34

8) References $\quad$ S36

9) ${ }^{1} \mathrm{H}$ and ${ }^{13} \mathrm{C}$ NMR spectra of the compounds $\quad$ S37 
1) General considerations and reagent information Unless otherwise stated, all reactions were carried out under air atmosphere in screw cap reaction tubes. All the solvents were bought from Aldrich in sure-seal bottle and were used as received. $\mathrm{Pd}(\mathrm{OAc})_{2}$ and other reagents were bought from Aldrich. The reactions were performed using IKA RCT basic stirrer equipped with oil bath for heating. For column chromatography, silica gel (100-200 mesh) from Finar Co. was used. A gradient elution using petroleum ether and ethyl acetate was performed based on Merck aluminum TLC sheets (silica gel 60F254). All directing groups attached to the unactivated alkenyl amide ${ }^{1}$ and alkynes ${ }^{2}$ were prepared according to the reported procedures in the literatures and the analytical data are in accord with the literature.

Analytical information All isolated compounds are characterized by ${ }^{1} \mathrm{H}$ NMR, ${ }^{13} \mathrm{C}$ NMR spectroscopy. In addition, all the compounds are further characterized by HRMS. Copies of ${ }^{1} \mathrm{H}$ $\mathrm{NMR}$ and ${ }^{13} \mathrm{C}$ NMR can be found in the supporting information. Nuclear magnetic resonance spectra were recorded either on a Bruker 500 or a $400 \mathrm{MHz}$ instrument. All ${ }^{1} \mathrm{H}$ NMR experiments are reported in units, parts per million ( $\mathrm{ppm})$, and were measured relative to the signals for residual chloroform $(7.26 \mathrm{ppm})$ in the deuterated solvent, unless otherwise stated. All ${ }^{13} \mathrm{C}$ NMR spectra were reported in ppm relative to deuteron chloroform (77.16 ppm), unless otherwise stated, and all were obtained with ${ }^{1} \mathrm{H}$ decoupling.

\section{2) General procedure for the synthesis of starting materials. ${ }^{1}$}

2a) Synthesis of N-(quinolin-8-yl)but-3-enamide (1a): Vinyl acetic acid (1.12 mL, 13 mmol) was charged into a $250 \mathrm{~mL} \mathrm{RB}$ flask containing $30 \mathrm{~mL}$ DCM. 8-Aminoquinoline $(1.44 \mathrm{~g}, 10$ $\mathrm{mmol})$, collidine $(2.6 \mathrm{~mL}, 20 \mathrm{mmol})$, and HATU (4.94 g, $13 \mathrm{mmol})$ were added sequentially, and the reaction was stirred at ambient temperature for $16 \mathrm{~h}$. The deep brown solution was diluted with EtOAc $(200 \mathrm{~mL})$, washed with sat. $\mathrm{NaHCO}_{3}(100 \mathrm{~mL} \times 2)$ and brine $(100 \mathrm{~mL} \times 1)$, and purified by column chromatography (10-15\% EtOAc in Hexanes) to afford $1.73 \mathrm{~g}(82 \%)$ yield of 1a as a yellow oil. Collidine was found to co-elute during column chromatography and was removed by repeated co-evaporation with large volumes of toluene with care to keep the temperature $\leq 35{ }^{\circ} \mathrm{C}$. Elevated temperatures may lead to isomerization to the conjugated alkene. ${ }^{1}$ 
2b) General procedure for the synthesis of $O$-tethered alkynes: ${ }^{2}$

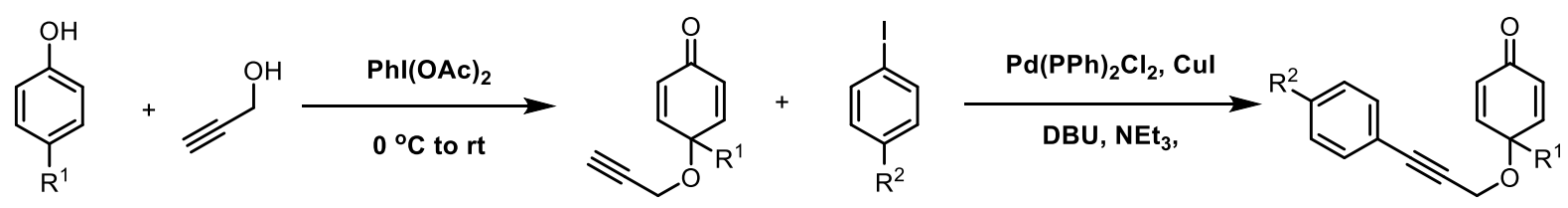

To a stirred solution of 4-substituted phenol $(1.0 \mathrm{mmol})$ in $1 \mathrm{~mL}$ of propargyl alcohol was added phenyliodine(III) diacetate $(1.5 \mathrm{mmol})$ in several portions at $0{ }^{\circ} \mathrm{C}$. The resulting reaction mixture was stirred at room temperature for overnight. Then the reaction mixture was diluted with water $(10 \mathrm{~mL})$ and extracted with ethyl acetate $(15 \mathrm{~mL} \times 3)$. The combined organic solvent was washed with brine $(15 \mathrm{~mL})$, dried $\left(\mathrm{Na}_{2} \mathrm{SO}_{4}\right)$, filtered, and concentrated in vacuo. The crude reaction mixture was purified by column chromatography (EtOAc/hexane) to give the Otethered terminal alkyne.

To a solution of $O$-tethered alkyne $(1.0 \mathrm{mmol})$ in degassed $\mathrm{Et}_{3} \mathrm{~N}(1 \mathrm{M}, 1 \mathrm{~mL})$ was added $\mathrm{Pd}\left(\mathrm{PPh}_{3}\right)_{2} \mathrm{Cl}_{2}$ (3 mol\%), $\mathrm{CuI}(1.5 \mathrm{~mol} \%)$ and aryl iodide $(1.2 \mathrm{mmol})$. The mixture was stirred at room temperature for 3-5 hours. The reaction was cooled to room temperature, water (20 $\mathrm{mL})$ was added, and the mixture was extracted with EtOAc $(3 \times 20 \mathrm{~mL})$. The combined organic solvent was washed with $10 \%$ aqueous $\mathrm{HCl}(10 \mathrm{~mL})$, dried $\left(\mathrm{Na}_{2} \mathrm{SO}_{4}\right)$, filtered, and concentrated in vacuo. The mixture was purified by column chromatography (EtOAc/hexane) to give aryl substituted alkynes high yields. ${ }^{2}$

2c) General procedure for the synthesis of 1,3-diynes: The symmetrical and unsymmetrical 1,3-diynes were prepared from the corresponding terminal alkynes according to procedures described in the literature. ${ }^{3}$

2d) General procedure for the synthesis of (bromoethynyl)benzene: The bromoethynyl) benzene were prepared from the phenyl acetylene according to procedures described in the literature. ${ }^{4}$ 
3) Optimization of the reaction conditions ${ }^{a}$
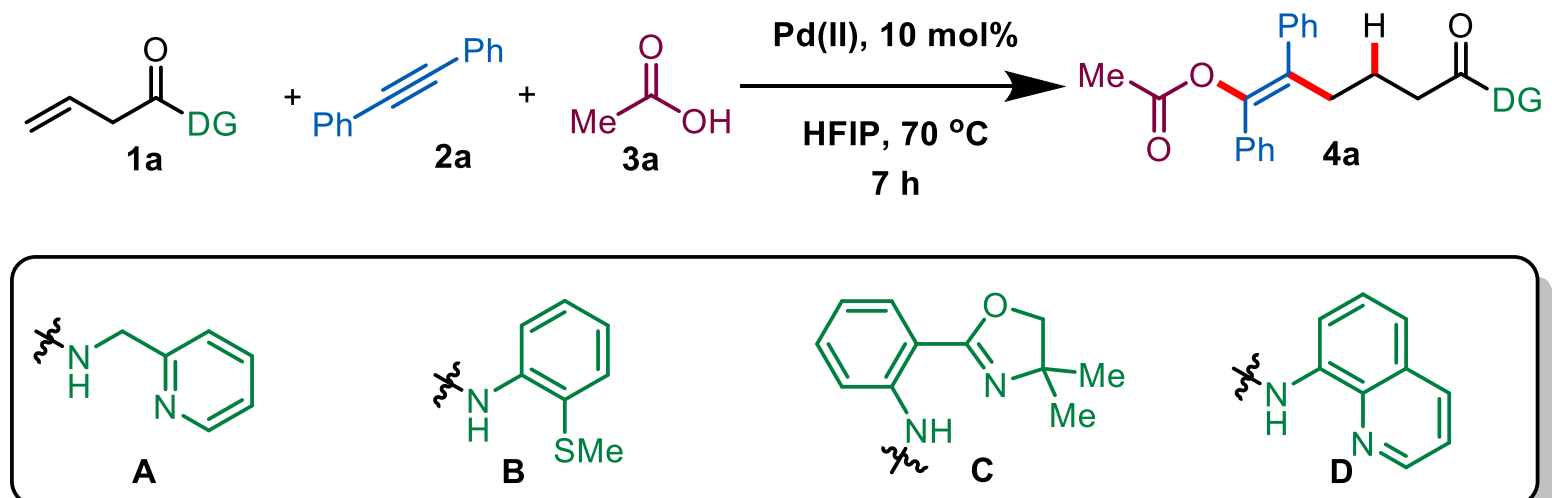

\begin{tabular}{|c|c|c|c|c|}
\hline Entry & Catalyst & Directing group & Solvent & Yield (\%) ${ }^{b}$ \\
\hline 1 & $\mathrm{Pd}(\mathrm{OAc})_{2}$ & A & EtOH & n.r. \\
\hline 2 & $\mathrm{Pd}(\mathrm{OAc})_{2}$ & B & EtOH & n.r. \\
\hline 3 & $\mathrm{Pd}(\mathrm{OAc})_{2}$ & C & EtOH & n.r. \\
\hline 4 & $\mathrm{Pd}(\mathrm{OAc})_{2}$ & D & EtOH & 30 \\
\hline 5 & $\mathrm{Pd}(\mathrm{OAc})_{2}$ & D & DCE & n.r. \\
\hline 6 & $\mathrm{Pd}(\mathrm{OAc})_{2}$ & D & Toluene & n.r. \\
\hline 7 & $\mathrm{Pd}(\mathrm{OAc})_{2}$ & D & $\mathrm{CH}_{3} \mathrm{NO}_{2}$ & n.r. \\
\hline 8 & $\mathrm{Pd}(\mathrm{OAc})_{2}$ & D & $\mathrm{CH}_{3} \mathrm{CN}$ & n.r. \\
\hline 9 & $\mathrm{Pd}(\mathrm{OAc})_{2}$ & D & THF & 10 \\
\hline 10 & $\mathrm{Pd}(\mathrm{OAc})_{2}$ & D & 1,4 dioxane & 5 \\
\hline 11 & $\mathrm{Pd}(\mathrm{OAc})_{2}$ & D & $t-\mathrm{Am}-\mathrm{OH}$ & 25 \\
\hline 12 & $\mathrm{Pd}(\mathrm{OAc})_{2}$ & D & TFE & 48 \\
\hline 13 & $\mathrm{Pd}(\mathrm{OAc})_{2}$ & D & HFIP & 70 \\
\hline $14^{c}$ & $\mathrm{Pd}(\mathrm{OAc})_{2}$ & D & HFIP & 78 \\
\hline $15^{d}$ & $\mathrm{Pd}(\mathrm{OAc})_{2}$ & D & HFIP & 90 \\
\hline 16 & $\mathrm{PdCl}_{2}$ & D & HFIP & 60 \\
\hline $17^{e}$ & $\mathrm{Pd}(\mathrm{OAc})_{2}$ & D & HFIP & 82 \\
\hline $18 f$ & $\mathrm{Pd}(\mathrm{OAc})_{2}$ & D & HFIP & 60 \\
\hline 19 & - & D & HFIP & n.r. \\
\hline $20 g$ & $\mathrm{Pd}(\mathrm{OAc})_{2}$ & D & HFIP & n.r. \\
\hline
\end{tabular}

Conditions: ${ }^{a} \mathbf{1 a}(0.1 \mathrm{mmol}), 2 \mathrm{a}(0.12 \mathrm{mmol}), 3 \mathbf{3 a}(0.2 \mathrm{mmol}) .{ }^{b} \mathrm{NMR}$ yield (using $1,3,5$-trimethoxy benzene as internal standard). ${ }^{c} \mathbf{3 a}(0.25 \mathrm{mmol}) .{ }^{d} \mathbf{3 a}(0.3 \mathrm{mmol}) .{ }^{e}$ Reaction at $50{ }^{\circ} \mathrm{C} . f \mathrm{Pd}(\mathrm{OAc})_{2}(5 \mathrm{~mol} \%) . g$ Without $\mathbf{3 a} \cdot$ n.r. $=$ no reaction

4) General procedure for " $\mathrm{Pd}(\mathrm{OAc})_{2}$ catalyzed trans-acetoxylation of alkynes followed by in situ regioselective addition to an unactivated alkene<smiles>[R]C#[N+][CH+][R]</smiles>

$2 a$

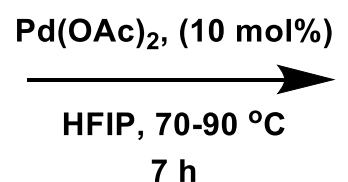<smiles>[R]C(=O)OC([R])=C([R])CCC(=O)NO</smiles> 
To an oven dried screw-cap reaction tube equipped with stir bar, $N$-(quinolin-8-yl)but-3enamide $(0.1 \mathrm{mmol})$, Alkyne $(0.12 \mathrm{mmol})$ and $\mathrm{Pd}(\mathrm{OAC})_{2}$ catalyst $(10 \mathrm{~mol} \%)$ were added sequentially, followed by HFIP $(1 \mathrm{~mL})$ and $\mathrm{RCOOH}$ (3 equiv.).The reaction mixture was heated for $6-12 \mathrm{hrs}$ at $70-90^{\circ} \mathrm{C}$. The completion of the reaction was confirmed by checking TLC under UV detector. Then, the organic phase was evaporated under reduced pressure and the product was purified by using silica-gel Column Chromatography (eluent : Hexane/Ethyl acetate $=9 / 1)$

\section{5a) Proposed reaction mechanism.}

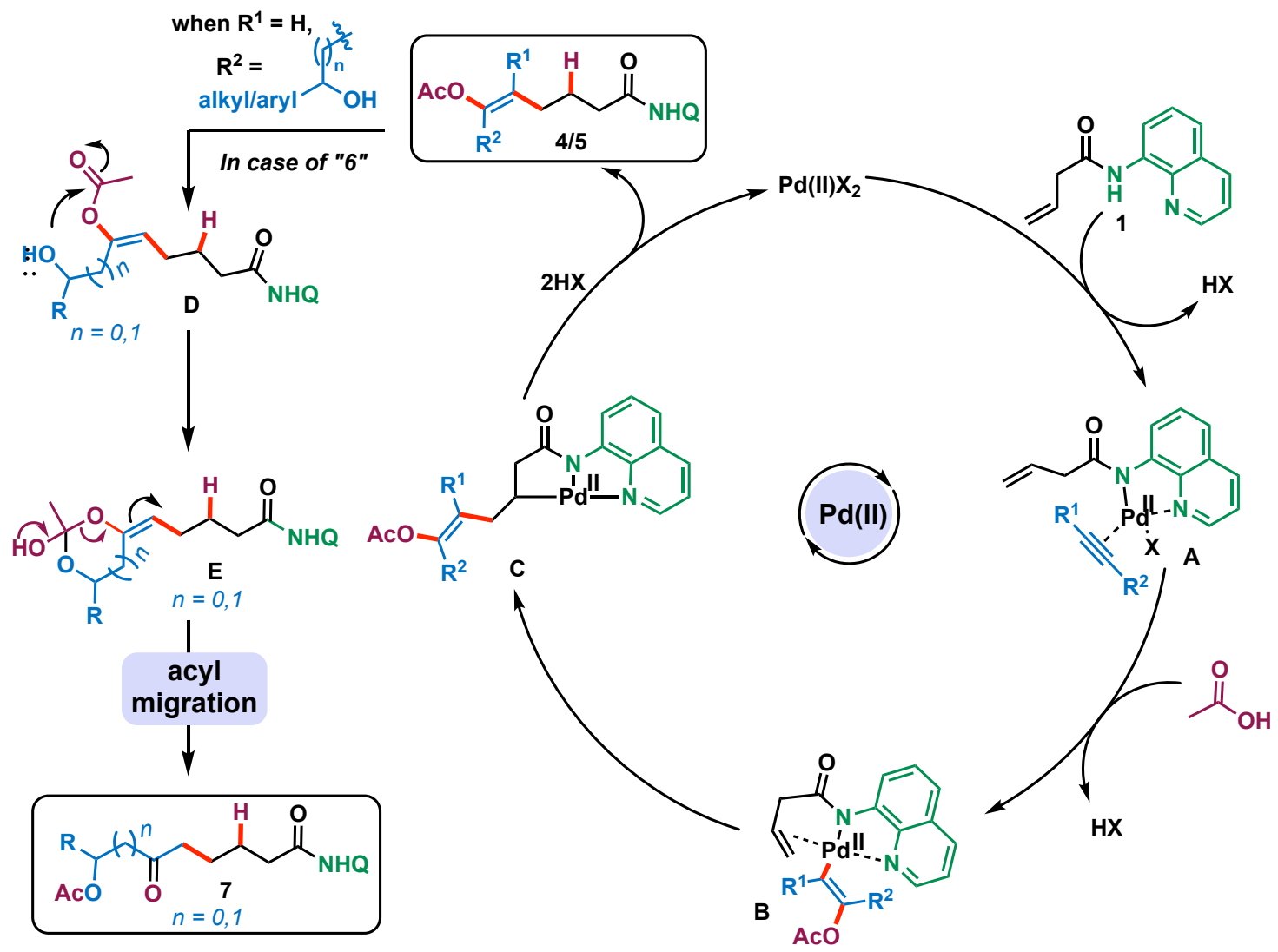


5b) Proposed formation of enone (7i) via cascade hydrocarbofunctionalization, acyl migration followed by $\mathrm{E} 1 \mathrm{cB}$ reaction<smiles>C=CCC(N)=O</smiles>

1a<smiles>CC(=O)O</smiles><smiles>C=C(CCC(=O)NO)CC(=O)CC</smiles>

$6 \mathbf{i}$

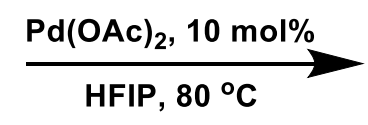

Hydrocarbofunctionalization

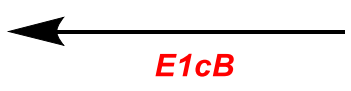

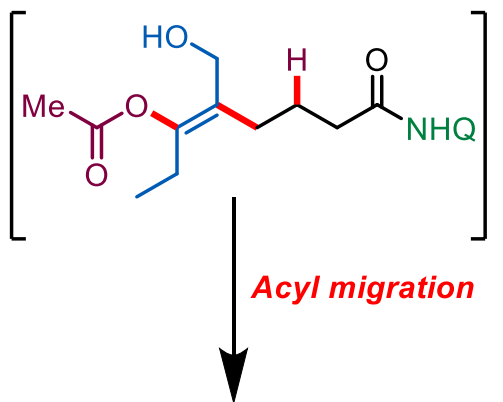

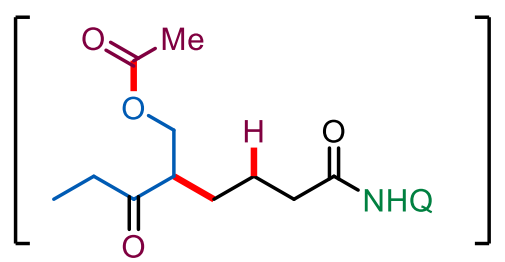

6) Characterization data of new compounds:<smiles>CC(=O)O/C(=C(\CCC(=O)NO)c1ccccc1)c1ccccc1</smiles>

$4 a, 88 \%$

(Z)-6-oxo-1,2-diphenyl-6-(quinolin-8-ylamino)hex-1-en-1-yl acetate(4a)

$39.7 \mathrm{mg}, 88 \%$ yield, white solid, m.p $=178-180^{\circ} \mathrm{C}$

$\boldsymbol{R}_{\boldsymbol{f}}: 0.32$ (hexane/ethyl acetate, $8: 2 \mathrm{v} / \mathrm{v}$ ).

IR (solid): $v$ 3362, 3046, 3026, 2935, 2842,2435, 1951, 1879, 1753,1685, 1642, 1588, 1537 , $1477,1426,1376,1335,1214,1055,935,571 \mathrm{~cm}^{-1}$.

${ }^{1}$ H NMR (400 MHz, CDCl $) \delta 9.68(\mathrm{~s}, 1 \mathrm{H}), 8.78(\mathrm{dd}, J=4.2,1.5 \mathrm{~Hz}, 1 \mathrm{H}), 8.70(\mathrm{dd}, J=7.0$, $1.7 \mathrm{~Hz}, 1 \mathrm{H}), 8.14(\mathrm{dd}, J=8.2,1.0 \mathrm{~Hz}, 1 \mathrm{H}), 7.53-7.46(\mathrm{~m}, 4 \mathrm{H}), 7.44(\mathrm{dd}, J=8.2,4.2 \mathrm{~Hz}, 1 \mathrm{H})$, $7.38-7.32(\mathrm{~m}, 4 \mathrm{H}), 7.31-7.25(\mathrm{~m}, 4 \mathrm{H}), 2.65-2.57(\mathrm{t}, J=7.8, \mathrm{~Hz}, 2 \mathrm{H}), 2.48(\mathrm{t}, J=7.6 \mathrm{~Hz}$, 2H), $1.90-1.84$ (m, 2H), 1.82 (s, 3H).

${ }^{13}$ C NMR (100 MHz, $\left.\mathbf{C D C l}_{3}\right) \delta 171.1,169.6,148.2,143.3,138.4,138.4,136.4,135.6,134.50$, $130.6,129.0,128.5,128.4,128.3,128.1,128.0,127.4,127.3,121.4,121.4,116.5,37.6,32.5$, 24.4, 20.8 .

HRMS (ESI-TOF) m/z: [M+Na $]^{+}$calcd for $\mathrm{C}_{29} \mathrm{H}_{26} \mathrm{~N}_{2} \mathrm{NaO}_{3} 473.1836$; Found 473.1830 . 
<smiles>CCCCCCCCCCC(=O)OC(C(=O)NO)=C(c1ccccc1)c1ccccc1</smiles>

4b, $89 \%$

\section{(Z)-6-oxo-1,2-diphenyl-6-(quinolin-8-ylamino)hex-1-en-1-yl propionate (4b)}

$41.4 \mathrm{mg}, 89 \%$ yield, yellow liquid.

$\boldsymbol{R}_{\boldsymbol{f}}: 0.35$ (hexane/ethyl acetate, $8: 2 \mathrm{v} / \mathrm{v}$ ).

IR (CHCl $\mathbf{3})$ : v 3350, 3051, 3010, 2920, 2851, 2425, 1889, 1755, 1707, 1685, 1640, 1590, 1470, $1446,1326,1215,1060,896,586 \mathrm{~cm}^{-1}$.

${ }^{1}$ H NMR (400 MHz, CDCl $)$ ) $\delta 9.68(\mathrm{~s}, 1 \mathrm{H}), 8.78(\mathrm{dd}, J=4.2,1.4,1 \mathrm{H}), 8.70(\mathrm{dd}, J=6.9,1.7$, $1 \mathrm{H}), 8.15(\mathrm{dd}, J=8.2,1.4,1 \mathrm{H}), 7.54-7.47(\mathrm{~m}, 4 \mathrm{H}), 7.44(\mathrm{dd}, J=8.3,4.2,1 \mathrm{H}), 7.38-7.31$ $(\mathrm{m}, 4 \mathrm{H}), 7.31-7.24(\mathrm{~m}, 4 \mathrm{H}), 2.64-2.57(\mathrm{~m}, 2 \mathrm{H}), 2.48(\mathrm{t}, J=7.6,2 \mathrm{H}), 2.08(\mathrm{q}, J=7.6,2 \mathrm{H})$, $1.93-1.82(\mathrm{~m}, 2 \mathrm{H}), 0.87(\mathrm{t}, J=7.6,3 \mathrm{H})$.

${ }^{13}$ C NMR (100 MHz, CDC13) $\delta 173.0,171.2,148.2,143.3,138.5,138.4,136.5,135.6,134.5$, $130.6,129.0,128.5,128.3,128.2,128.0,127.5,127.3,121.7,121.5,116.5,37.6,32.5,27.5$, 24.4, 8.9.

HRMS (ESI-TOF) m/z: [M+H $]^{+}$calcd for $\mathrm{C}_{30} \mathrm{H}_{29} \mathrm{~N}_{2} \mathrm{O}_{3} 465.2173$; Found 465.2169.<smiles>O=C(CCC(=C(OC(=O)CCCc1ccccc1)c1ccccc1)c1ccccc1)NO</smiles>

4c, $80 \%$

\section{(Z)-6-oxo-1,2-diphenyl-6-(quinolin-8-ylamino)hex-1-en-1-yl 4-phenylbutanoate (4c)}

$44.4 \mathrm{mg}, 80 \%$ yield, light yellow liquid.

$\boldsymbol{R}_{\boldsymbol{f}}: 0.30$ (hexane/ethyl acetate, $8: 2 \mathrm{v} / \mathrm{v}$ ).

IR (CHCl) $)$ v 3472, 3056, 3018, 2964, 2865, 2433, 2400, 1889, 1743, 1684, 1598, 1527, 1487, $1424,1386,1325,1217,1131,1065,925,826,562 \mathrm{~cm}^{-1}$.

${ }^{1}$ H NMR (500 MHz, CDCl $) \delta 9.74(\mathrm{~s}, 1 \mathrm{H}), 8.82(\mathrm{~d}, J=3.9,1 \mathrm{H}), 8.77$ (d, $\left.J=7.3,1 \mathrm{H}\right), 8.17$ $(\mathrm{d}, J=8.2,1 \mathrm{H}), 7.58-7.50(\mathrm{~m}, 4 \mathrm{H}), 7.46(\mathrm{dd}, J=8.2,4.2,1 \mathrm{H}), 7.40(\mathrm{~d}, J=4.3,4 \mathrm{H}), 7.37-$ $7.27(\mathrm{~m}, 6 \mathrm{H}), 7.21(\mathrm{t}, J=7.2,1 \mathrm{H}), 7.07(\mathrm{~d}, J=7.5,2 \mathrm{H}), 2.70-2.64(\mathrm{~m}, 2 \mathrm{H}), 2.53(\mathrm{t}, J=7.5$, $2 \mathrm{H}), 2.43(\mathrm{t}, J=7.6,2 \mathrm{H}), 2.15(\mathrm{t}, J=7.3,2 \mathrm{H}), 1.98-1.89(\mathrm{~m}, 2 \mathrm{H}), 1.76-1.65(\mathrm{~m}, 2 \mathrm{H})$. 
${ }^{13}$ C NMR (125 MHz, CDC13) $\delta 171.9,171.1,148.1,143.2,141.3,138.4,138.3,136.4,135.5$, $134.5,130.7,129.0,128.5,128.4,128.3,128.3,128.3,128.2,127.9,127.4,127.3,126.0,121.6$, $121.4,116.4,37.5,34.7,33.3,32.5,26.1,24.3$.

HRMS (ESI-TOF) m/z: [M+Na $]^{+}$calcd for $\mathrm{C}_{37} \mathrm{H}_{34} \mathrm{~N}_{2} \mathrm{NaO}_{3}$ 577.2462; Found 577.2458.<smiles>O=C(CCC(=C(P)OC(=O)CCC1CCCCC1)c1ccccc1)NO</smiles>

\section{(Z)-6-oxo-1,2-diphenyl-6-(quinolin-8-ylamino)hex-1-en-1-yl 3-cyclohexylpropanoate}

$\underline{(4 d)}$

$45.9 \mathrm{mg}, 84 \%$ yield, semi solid.

$\boldsymbol{R}_{\boldsymbol{f}}: 0.32$ (hexane/ethyl acetate, $8: 2 \mathrm{v} / \mathrm{v}$ ).

IR (CHCl $): v$ 3352, 3056, 3016, 2925, 2852, 2667, 2435, 2400, 1951, 1889, 1773, 17271685 , $1644,1598,1527,1487,14246,1386,1325,1215,1065,925,826,581 \mathrm{~cm}^{-1}$.

${ }^{1}$ H NMR (500 MHz, CDCl $) \delta 9.71(\mathrm{~s}, 1 \mathrm{H}), 8.85$ - $8.77(\mathrm{~m}, 1 \mathrm{H}), 8.72(\mathrm{~d}, J=7.0,1 \mathrm{H}), 8.17$ $(\mathrm{d}, J=8.2,1 \mathrm{H}), 7.55-7.49(\mathrm{~m}, 4 \mathrm{H}), 7.46(\mathrm{dd}, J=8.2,4.2,1 \mathrm{H}), 7.40-7.34(\mathrm{~m}, 4 \mathrm{H}), 7.32-$ $7.26(\mathrm{~m}, 4 \mathrm{H}), 2.68-2.58(\mathrm{~m}, 2 \mathrm{H}), 2.51(\mathrm{t}, J=7.5,2 \mathrm{H}), 2.10(\mathrm{t}, J=7.7,2 \mathrm{H}), 1.93-1.84(\mathrm{~m}$, $2 \mathrm{H}), 1.76-1.50(\mathrm{~m}, 7 \mathrm{H}), 1.28-1.09(\mathrm{~m}, 4 \mathrm{H}), 0.78-0.68(\mathrm{~m}, 2 \mathrm{H})$.

${ }^{13}$ C NMR (125 MHz, CDCl 3$) \delta 172.5,171.2,148.1,143.2,138.4,138.3,136.4,135.6,134.4$, $130.5,129.0,128.3,128.3,128.2$, 128.2 , 127.9, 127.4, 127.2, 121.6, 121.4, 116.6, 37.5, 36.7, $32.8,32.4,31.8,31.5,26.5,26.1,24.3$.

HRMS (ESI-TOF) m/z: [M+H] $]^{+}$calcd for $\mathrm{C}_{36} \mathrm{H}_{39} \mathrm{~N}_{2} \mathrm{O}_{3}$ 547.2955; Found 547.2954.<smiles>C=CCC(=O)O/C(Pc1ccccc1)=C(/CCC(=O)NO)c1ccccc1</smiles>

$4 e, 86 \%$

(Z)-6-ox0-1,2-diphenyl-6-(quinolin-8-ylamino)hex-1-en-1-yl but-3-enoate (4e)

$41.0 \mathrm{mg}, 86 \%$ yield, liquid.

$\boldsymbol{R}_{\boldsymbol{f}}: 0.30$ (hexane/ethyl acetate, $8: 2 \mathrm{v} / \mathrm{v}$ ).

IR (CHCl $\mathbf{3})$ : v 3362, 3051, 3026, 2922, 2862, 2657, 2431,1951, 1885, 1763, 1737 1684, 1634, $1588,1521,1482,1424,1383,1324,1211,1060,920,822,582 \mathrm{~cm}^{-1}$. 
${ }^{1}$ H NMR (400 MHz, CDCl $) \delta 9.69(\mathrm{~s}, 1 \mathrm{H}), 8.78(\mathrm{dd}, J=4.2,1.6,1 \mathrm{H}), 8.71(\mathrm{dd}, J=7.1,1.7$, $1 \mathrm{H}), 8.14(\mathrm{dt}, J=8.3,4.1,1 \mathrm{H}), 7.53-7.45(\mathrm{~m}, 4 \mathrm{H}), 7.42(\mathrm{dd}, J=8.3,4.2,1 \mathrm{H}), 7.39-7.32(\mathrm{~m}$, 4H), $7.32-7.25(\mathrm{~m}, 4 \mathrm{H}), 5.68-5.52(\mathrm{~m}, 1 \mathrm{H}), 5.01(\mathrm{dd}, \mathrm{J}=10.2,1.3 \mathrm{~Hz}, 1 \mathrm{H}), 4.96(\mathrm{dd}, \mathrm{J}=$ 17.1, $1.4 \mathrm{~Hz}, 1 \mathrm{H}), 2.85(\mathrm{~d}, J=6.9,2 \mathrm{H}), 2.67-2.58(\mathrm{~m}, 2 \mathrm{H}), 2.48(\mathrm{t}, J=7.6,2 \mathrm{H}), 1.94-1.80$ $(\mathrm{m}, 2 \mathrm{H})$.

${ }^{13}$ C NMR (100 MHz, CDCl $) \delta 171.1,170.0,148.1,143.2,138.3,136.4,135.3,134.4,130.8$, $129.5,129.0,128.5,128.3,128.3,128.2$, 127.9, 127.4, 127.3, 121.6, 121.4, 118.7, 116.4, 38.8, $37.5,32.4,24.3$.

HRMS (ESI-TOF) m/z: [M+Na $]^{+}$calcd for $\mathrm{C}_{31} \mathrm{H}_{28} \mathrm{~N}_{2} \mathrm{NaO}_{3} 499.1992$; Found 499.1986.<smiles>O=C(CCC(=C(P)OC(=O)C(c1ccccc1)c1ccccc1)c1ccccc1)NO</smiles>

\section{(Z)-6-oxo-1,2-diphenyl-6-(quinolin-8-ylamino)hex-1-en-1-yl 2,2-diphenylacetate (4f)}

$51.1 \mathrm{mg}, 87 \%$ yield, liquid.

$\boldsymbol{R}_{\boldsymbol{f}}: 0.31$ (hexane/ethyl acetate, $8: 2 \mathrm{v} / \mathrm{v}$ ).

IR (CHCl $\mathbf{3})$ : $\mathbf{~ 3 3 7 2 , ~ 3 0 4 6 , ~ 3 0 1 4 , ~ 2 9 2 1 , ~ 2 8 4 0 , ~ 2 6 5 6 , ~ 2 4 4 6 , ~ 2 4 2 6 , ~ 1 9 4 8 , ~ 1 8 8 2 , ~ 1 7 6 8 , ~} 1739$ 1679, $1633,1589,1531,1478,1426,1379,1330,1220,1058,930,831,590 \mathrm{~cm}^{-1}$.

${ }^{1}$ H NMR (400 MHz, CDCl $) \delta 9.69(\mathrm{~s}, 1 \mathrm{H}), 8.77(\mathrm{dd}, J=4.2,1.7,1 \mathrm{H}), 8.73(\mathrm{dd}, J=7.2,1.7$, $1 \mathrm{H}), 8.13(\mathrm{dd}, J=8.3,1.7,1 \mathrm{H}), 7.55-7.46(\mathrm{~m}, 2 \mathrm{H}), 7.43(\mathrm{dd}, J=8.3,4.2,1 \mathrm{H}), 7.39-7.34$ (m, 2H), $7.33-7.28(\mathrm{~m}, 5 \mathrm{H}), 7.26-7.17(\mathrm{~m}, 9 \mathrm{H}), 6.99-6.93(\mathrm{~m}, 4 \mathrm{H}), 4.85(\mathrm{~s}, 1 \mathrm{H}), 2.80-$ $2.57(\mathrm{~m}, 2 \mathrm{H}), 2.48(\mathrm{t}, J=7.6,2 \mathrm{H}), 1.92-1.83(\mathrm{~m}, 2 \mathrm{H})$.

${ }^{13}$ C NMR (100 MHz, $\left.\mathbf{C D C l}_{3}\right) \delta 171.1,170.7,148.1,143.3,138.3,138.2,138.0,136.4,134.9$, $134.5,130.9,129.0,128.7,128.5,128.4,128.2,128.1,127.9,127.4,127.3,127.1,121.6,121.4$, $116.4,56.8,37.5,32.6,24.2$.

HRMS (ESI-TOF) m/z: [M+Na $]^{+}$calcd for $\mathrm{C}_{41} \mathrm{H}_{34} \mathrm{~N}_{2} \mathrm{NaO}_{3}$ 625.2462; Found 625.2463.<smiles>CC(C)(C)C(=O)O/C(=C(\CCC(=O)NO)c1ccccc1)c1ccccc1</smiles>

4g, $55 \%$

(Z)-6-0xo-1,2-diphenyl-6-(quinolin-8-ylamino)hex-1-en-1-yl pivalate (4g)

$27.1 \mathrm{mg}, 55 \%$ yield, liquid. 
$\boldsymbol{R}_{\boldsymbol{f}}: 0.33$ (hexane/ethyl acetate, $8: 2 \mathrm{v} / \mathrm{v}$ ).

IR (CHCl $)$ : v 3366, 3061, 3020, 2922, 2848, 2661, 2437, 1956, 1881, 1763, 1739, 1681, 1642, $1591,1520,1472,1446,1382,1321,1210,1062,921,822,584 \mathrm{~cm}^{-1}$.

${ }^{1}$ H NMR (400 MHz, CDCl $)$ ) $\delta 9.67(\mathrm{~s}, 1 \mathrm{H}), 8.78(\mathrm{dd}, J=4.2,1.5,1 \mathrm{H}), 8.69$ (dd, $J=6.9,1.9$, $1 \mathrm{H}), 8.15(\mathrm{dd}, J=8.2,1.5,1 \mathrm{H}), 7.52-7.43(\mathrm{~m}, 6 \mathrm{H}), 7.32-7.23(\mathrm{~m}, 7 \mathrm{H}), 2.66-2.55(\mathrm{~m}, 2 \mathrm{H})$, $2.48(\mathrm{t}, J=7.6,2 \mathrm{H}), 1.94-1.81(\mathrm{~m}, 2 \mathrm{H}), 0.90(\mathrm{~s}, 9 \mathrm{H})$.

${ }^{13}$ C NMR (125 MHz, CDCl 3$) \delta 176.7,171.2,148.2,143.4,138.6,138.4,136.5,135.6,134.6$, $130.5,128.8,128.5,128.4,128.3,128.2,128.0,127.5,127.2,121.7,121.5,116.6,38.6,37.7$, 32.6, 26.8, 24.3.

HRMS (ESI-TOF) m/z: [M+Na $]^{+}$calcd for $\mathrm{C}_{32} \mathrm{H}_{32} \mathrm{~N}_{2} \mathrm{NaO}_{3}$ 515.2305; Found 515.2309.<smiles>O=C(CC/C(=C(/OC(=O)C1CCC1)c1ccccc1)c1ccccc1)NO</smiles>

4h, $78 \%$

(Z)-6-oxo-1,2-diphenyl-6-(quinolin-8-ylamino)hex-1-en-1-yl cyclobutanecarboxylate (4h) $38.3 \mathrm{mg}, 78 \%$ yield, yellow liquid.

$\boldsymbol{R}_{\boldsymbol{f}}: 0.32$ (hexane/ethyl acetate, $8: 2 \mathrm{v} / \mathrm{v}$ ).

IR (CHCl $):$ v 3355, 3059, 3026, 2921, 2848, 2661, 2432, 1951, 1878, 1740, 1688, 1641, 1582 , $1534,1474,1436,1381,1355,1220,1034,920,831,575 \mathrm{~cm}^{-1}$.

${ }^{1}$ H NMR (400 MHz, CDCl $\left.)_{3}\right) 9.69(\mathrm{~s}, 1 \mathrm{H}), 8.76(\mathrm{dd}, J=4.1,1.4,1 \mathrm{H}), 8.69(\mathrm{dd}, J=6.9,1.7$, $1 \mathrm{H}), 8.11(\mathrm{dd}, J=8.3,1.4,1 \mathrm{H}), 7.52-7.44(\mathrm{~m}, 4 \mathrm{H}), 7.40(\mathrm{dd}, J=8.3,4.2,1 \mathrm{H}), 7.38-7.31$ $(\mathrm{m}, 4 \mathrm{H}), 7.30-7.22(\mathrm{~m}, 4 \mathrm{H}), 2.97-2.86(\mathrm{~m}, 1 \mathrm{H}), 2.66-2.58(\mathrm{~m}, 2 \mathrm{H}), 2.49(\mathrm{t}, J=7.5,2 \mathrm{H})$, $1.99-1.85(\mathrm{~m}, 8 \mathrm{H})$.

${ }^{13}$ C NMR (100 MHz, $\left.\mathbf{C D C l}_{3}\right) \delta 173.6,171.3,148.1,143.1,138.3,138.2,136.4,135.5,134.3$, $130.5,128.9,128.3,128.2,127.9,127.3,127.1,121.5,116.7,37.8,37.4,32.4,24.6,24.2$, 18.2. HRMS (ESI-TOF) m/z: [M+H] $]^{+}$calcd for $\mathrm{C}_{32} \mathrm{H}_{31} \mathrm{~N}_{2} \mathrm{O}_{3} 491.2329$; Found 491.2327.<smiles>O=C(CCC(=C(OC(=O)CC12CC3CC(CC(C3)C1)C2)c1ccccc1)c1ccccc1)NO</smiles>

$4 \mathbf{i}, 70 \%$

(Z)-6-oxo-1,2-diphenyl-6-(quinolin-8-ylamino)hex-1-en-1-yl 2-((3r,5r,7r)-adamantan-1yl)acetate (4i) 
$40.9 \mathrm{mg}, 70 \%$ yield, liquid.

$\boldsymbol{R}_{\boldsymbol{f}}: 0.34$ (hexane/ethyl acetate, $8: 2 \mathrm{v} / \mathrm{v}$ ).

IR (CHCl $)$ : v 3368, 3047, 3022, 2931, 2857, 2657, 2436, 2425, 1955, 1883, 1781, 1740 1688, $1647,1590,1530,1488,1469,1370,1330,1235,1055,934,827,588 \mathrm{~cm}^{-1}$.

${ }^{1}$ H NMR (400 MHz, $\left.\mathbf{C D C l}_{3}\right) \delta 9.66(\mathrm{~s}, 1 \mathrm{H}), 8.79(\mathrm{dd}, J=4.2,1.5,1 \mathrm{H}), 8.68(\mathrm{dd}, J=6.8,2.0$, 1H), 8.15 (dd, $J=8.3,1.5,1 \mathrm{H}), 7.53-7.47$ (m, 4H), 7.45 (dd, $J=8.3,4.3,1 \mathrm{H}), 7.37-7.31$ (m, 4H), $7.29-7.23(\mathrm{~m}, 4 \mathrm{H}), 2.60-2.53(\mathrm{~m}, 2 \mathrm{H}), 2.46(\mathrm{t}, J=7.6,2 \mathrm{H}), 1.87-1.77(\mathrm{~m}, 7 \mathrm{H})$, $1.64-1.56(\mathrm{~m}, 4 \mathrm{H}), 1.47(\mathrm{~d}, J=11.7,3 \mathrm{H}), 1.30(\mathrm{~d}, J=2.0,6 \mathrm{H})$.

${ }^{13}$ C NMR (100 MHz, CDCl 3$) \delta 171.2,170.3,148.2,143.4,138.6,138.4,136.5,135.8,134.6$, $130.7,129.3,128.5,128.4,128.2,128.0,127.5,127.2,121.7,121.4,116.5,48.4,42.0,37.7$, $36.7,32.9,32.8,28.6,24.3$.

HRMS (ESI-TOF) m/z: [M+H] $]^{+}$calcd for $\mathrm{C}_{39} \mathrm{H}_{41} \mathrm{~N}_{2} \mathrm{O}_{3}$ 585.3112; Found 585.3120.<smiles>O=C(CCC(=C(OC(=O)C1CCCO1)c1ccccc1)c1ccccc1)NO</smiles>

$4 \mathbf{j}, 80 \%$

(Z)-6-oxo-1,2-diphenyl-6-(quinolin-8-ylamino)hex-1-en-1-yl tetrahydrofuran-2-

\section{carboxylate (4i)}

$40.5 \mathrm{mg}, 80 \%$ yield, light brown liquid.

$\boldsymbol{R}_{\boldsymbol{f}}: 0.25$ (hexane/ethyl acetate, 8:2 v/v).

IR (CHCl $):$ v 3372, 3048, 3021, 2922, 2847, 2659, 2431, 2405, 1947, 1878, 1773, 1747 1683, $1640,1600,1540,1486,1424,1387,1343,1225,1211,1050,922,823,571 \mathrm{~cm}^{-1}$.

${ }^{1}$ H NMR (400 MHz, CDCl $) \delta 9.67$ (s, 1H), 8.77 (dd, $\left.J=4.2,1.5,1 \mathrm{H}\right), 8.69$ (dd, $J=6.9,1.9$, $1 \mathrm{H}), 8.13(\mathrm{dd}, J=8.3,1.5,1 \mathrm{H}), 7.52-7.46(\mathrm{~m}, 4 \mathrm{H}), 7.43$ (dd, $J=8.3,4.2,1 \mathrm{H}), 7.38-7.29$ (m, 4H), $7.29-7.21(\mathrm{~m}, 4 \mathrm{H}), 4.25(\mathrm{dd}, J=8.6,4.9,1 \mathrm{H}), 3.85-3.67(\mathrm{~m}, 2 \mathrm{H}), 2.65-2.53(\mathrm{~m}$, $2 \mathrm{H}), 2.47(\mathrm{t}, J=7.6,2 \mathrm{H}), 1.91-1.85(\mathrm{~m}, 3 \mathrm{H}), 1.65(\mathrm{dt}, J=14.2,6.1,1 \mathrm{H}), 1.52(\mathrm{tt}, J=12.7$, $6.5,1 \mathrm{H}), 1.35-1.27(\mathrm{~m}, 2 \mathrm{H})$.

${ }^{13}$ C NMR (100 MHz, $\left.\mathbf{C D C l}_{3}\right) \delta 171.7,171.1,148.2,142.9,138.3,138.3,136.4,135.0,134.5$, $131.1,129.1,128.6,128.4,128.3,128.3,128.0,127.4,127.4,121.6,121.4,116.5,76.4,69.2$, $37.5,32.7,29.4,24.4,24.2$.

HRMS (ESI-TOF) m/z: [M+Na $]^{+}$calcd for $\mathrm{C}_{32} \mathrm{H}_{30} \mathrm{~N}_{2} \mathrm{NaO}_{4}$ 529.2098; Found 529.2095. 
<smiles>C=C(C)C(=O)O/C(=C(\CCC(=O)NO)c1ccccc1)c1ccccc1</smiles>

4k, $81 \%$

\section{(Z)-6-0xo-1,2-diphenyl-6-(quinolin-8-ylamino)hex-1-en-1-yl methacrylate (4k)}

$38.6 \mathrm{mg}, 81 \%$ yield, yellow liquid.

$\boldsymbol{R}_{\boldsymbol{f}}: 0.29$ (hexane/ethyl acetate, $8: 2 \mathrm{v} / \mathrm{v}$ ).

IR (CHCl $\mathbf{3}):$ v 3369, 3060, 3021, 2931, 2851, 2677, 2445, 2415, 1941, 1879, 1763, 1737 1687, $1634,1588,1517,1477,1426,1376,1335,1225,1055,935,816,583 \mathrm{~cm}^{-1}$.

${ }^{1}$ H NMR (400 MHz, CDCl $) \delta 9.68(\mathrm{~s}, 1 \mathrm{H}), 8.79(\mathrm{dd}, J=4.1,1.3,1 \mathrm{H}), 8.69(\mathrm{dd}, J=6.9,1.8$, $1 \mathrm{H}), 8.16(\mathrm{dd}, J=8.2,1.4,1 \mathrm{H}), 7.54-7.47(\mathrm{~m}, 4 \mathrm{H}), 7.45(\mathrm{dd}, J=8.3,4.2,1 \mathrm{H}), 7.33-7.30(\mathrm{~m}$, $4 \mathrm{H}), 7.29-7.22(\mathrm{~m}, 4 \mathrm{H}), 5.88(\mathrm{~s}, 1 \mathrm{H}), 5.41(\mathrm{~s}, 1 \mathrm{H}), 2.69-2.56(\mathrm{~m}, 2 \mathrm{H}), 2.48(\mathrm{t}, J=7.6,2 \mathrm{H})$, $1.92-1.82(\mathrm{~m}, 2 \mathrm{H}), 1.72(\mathrm{~s}, 3 \mathrm{H})$.

${ }^{13}$ C NMR (100 MHz, CDCl $\left._{3}\right) \delta 171.2,166.1,148.2,143.4,138.4,138.4,136.5,135.9,135.6$, 134.6, 130.6, 129.0, 128.5, 128.3, 128.3, 128.1, 128.0, 127.6, 127.3, 126.4, 121.7, 121.5, 116.6, $37.7,32.5,24.4,18.3$.

HRMS (ESI-TOF) m/z: [M+H] $]^{+}$calcd for $\mathrm{C}_{31} \mathrm{H}_{29} \mathrm{~N}_{2} \mathrm{O}_{3}$ 477.2173; Found 477.2178.<smiles>O=C(CCC(=C(OC(=O)C=Cc1ccccc1)c1ccccc1)c1ccccc1)NO</smiles>

$4 \mathbf{l}, 70 \%$

\section{(Z)-6-oxo-1,2-diphenyl-6-(quinolin-8-ylamino)hex-1-en-1-yl cinnamate (4l)}

$37.7 \mathrm{mg}, 70 \%$ yield, light yellow liquid.

$\boldsymbol{R}_{\boldsymbol{f}}: 0.26$ (hexane/ethyl acetate, $8: 2 \mathrm{v} / \mathrm{v}$ ).

IR (CHCl $\mathbf{3})$ : v 3367, 3047, 3026, 2927, 2843, 2671, 2438, 2407, 1939, 1878, 1763, 17391683 , $1654,1600,1537,1487,1388,1327,1218,1066,927,820,577 \mathrm{~cm}^{-1}$.

${ }^{1}$ H NMR (400 MHz, CDCl $)_{3} \delta 9.70(\mathrm{~s}, 1 \mathrm{H}), 8.79(\mathrm{dd}, J=4.2,1.6,1 \mathrm{H}), 8.72(\mathrm{dd}, J=7.1,1.5$, $1 \mathrm{H}), 8.15(\mathrm{dd}, J=8.3,1.5,1 \mathrm{H}), 7.58-7.47(\mathrm{~m}, 5 \mathrm{H}), 7.44(\mathrm{dd}, J=8.3,4.2,1 \mathrm{H}), 7.42-7.37$ $(\mathrm{m}, 4 \mathrm{H}), 7.37-7.30(\mathrm{~m}, 6 \mathrm{H}), 7.30-7.21(\mathrm{~m}, 3 \mathrm{H}), 6.26(\mathrm{~d}, J=16.0,1 \mathrm{H}), 2.72-2.61(\mathrm{~m}, 2 \mathrm{H})$, $2.51(\mathrm{t}, J=7.5,2 \mathrm{H}), 1.96-1.85(\mathrm{~m}, 2 \mathrm{H})$.

${ }^{13}$ C NMR (100 MHz, CDCl $) \delta$ 171.2, 165.6, 148.2, 145.9, 143.2, 138.4, 138.3, 136.4, 135.7, $134.5,134.3,130.7,130.5,129.0,128.9,128.5,128.4,128.4,128.2,128.2,128.0,127.5,127.3$, $121.7,121.5,117.3,116.5,37.7,32.5,24.5$. 
HRMS (ESI-TOF) m/z: [M+Na $]^{+}$calcd for $\mathrm{C}_{36} \mathrm{H}_{30} \mathrm{~N}_{2} \mathrm{NaO}_{3}$ 561.2149; Found 561.2150.<smiles>O=C(CC(CC(=O)OC(=O)c1ccccc1)c1ccccc1)NO</smiles>

(Z)-6-oxo-1,2-diphenyl-6-(quinolin-8-ylamino)hex-1-en-1-yl benzoate (4m)

$40.0 \mathrm{mg}, 78 \%$ yield, white solid, m.p $=190-193^{\circ} \mathrm{C}$.

$\boldsymbol{R}_{\boldsymbol{f}}: 0.30$ (hexane/ethyl acetate, $8: 2 \mathrm{v} / \mathrm{v}$ ).

IR(CHCl $):$ v 3363, 3053, 3027, 2929, 2854, 2663, 2420, 1949, 1878, 1762, 1738 1682, 1654, $1588,1560,1527,1487,1455,1426,1386,1325,1226,1055,928,830,588 \mathrm{~cm}^{-1}$.

${ }^{1}$ H NMR (400 MHz, CDCl $) \delta 9.70(\mathrm{~s}, 1 \mathrm{H}), 8.80(\mathrm{dd}, J=4.2,1.6,1 \mathrm{H}), 8.70(\mathrm{dd}, J=6.9,1.9$, $1 \mathrm{H}), 8.16(\mathrm{dd}, J=8.3,1.5,1 \mathrm{H}), 7.89-7.77(\mathrm{~m}, 2 \mathrm{H}), 7.58-7.44(\mathrm{~m}, 6 \mathrm{H}), 7.39-7.23(\mathrm{~m}, 8 \mathrm{H})$, $7.17(\mathrm{t}, J=7.4,1 \mathrm{H}), 2.81-2.63(\mathrm{~m}, 2 \mathrm{H}), 2.51(\mathrm{t}, J=7.6,2 \mathrm{H}), 1.95-1.84(\mathrm{~m}, 2 \mathrm{H})$.

${ }^{13}$ C NMR (100 MHz, CDCl 3$) \delta 171.3,165.3,148.2,143.4,138.5,136.5,135.6,134.6,133.1$, $130.9,130.3,130.0,129.1,128.5,128.4,128.2$, 128.1, 127.6, 127.3, 121.7, 121.5, 116.6, 37.7, 32.6, 24.5.

HRMS (ESI-TOF) m/z: [M+H] $]^{+}$calcd for $\mathrm{C}_{34} \mathrm{H}_{29} \mathrm{~N}_{2} \mathrm{O}_{3}$ 513.2173; Found 513.2174.

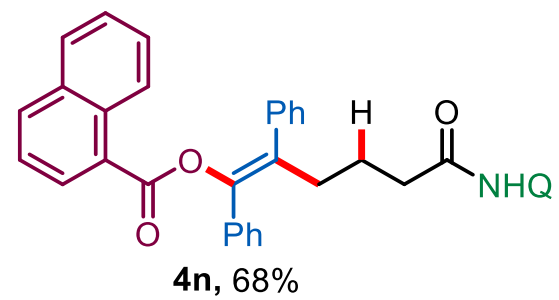

\section{(Z)-6-0xo-1,2-diphenyl-6-(quinolin-8-ylamino)hex-1-en-1-yl 1-naphthoate (4n)}

$38.3 \mathrm{mg}, 68 \%$ yield, white solid, m.p $=191-192^{\circ} \mathrm{C}$.

$\boldsymbol{R}_{\boldsymbol{f}}: 0.30$ (hexane/ethyl acetate, $8: 2 \mathrm{v} / \mathrm{v}$ ).

IR (CHCl $)$ : v 3365, 3060, 3022, 2922, 2857, 2664, 2431, 2422, 1953, 1885, 1770, 1735, 1683, $1655,1588,1523,1455,1424,1378,1322,1220,1060,921,827,584 \mathrm{~cm}^{-1}$.

${ }^{1}$ H NMR (400 MHz, CDCl $) \delta 9.74(\mathrm{~s}, J=19.4,1 \mathrm{H}), 8.80(\mathrm{dd}, J=4.0,1.2,1 \mathrm{H}), 8.75(\mathrm{~d}, J=$ $6.7,1 \mathrm{H}), 8.46(\mathrm{dd}, J=6.1,3.6,1 \mathrm{H}), 8.14(\mathrm{dd}, J=8.2,1.2,1 \mathrm{H}), 7.92(\mathrm{~d}, J=8.2,1 \mathrm{H}), 7.79(\mathrm{dd}$, $J=12.3,5.5,2 \mathrm{H}), 7.66(\mathrm{~d}, J=6.9,2 \mathrm{H}), 7.55-7.42(\mathrm{~m}, 7 \mathrm{H}), 7.38-7.28(\mathrm{~m}, 5 \mathrm{H}), 7.23(\mathrm{dd}, J$ $=14.7,7.3,1 \mathrm{H}), 2.81-2.69(\mathrm{~m}, 2 \mathrm{H}), 2.57(\mathrm{t}, J=7.5,2 \mathrm{H}), 2.04-1.93(\mathrm{~m}, 2 \mathrm{H})$. 
${ }^{13}$ C NMR (100 MHz, CDCl 3$) \delta 171.2,166.1,148.2,143.5,138.6,138.4,136.4,135.6,134.5$, $133.7,133.4,131.2,131.1,130.2,129.0,128.5,128.5,128.4,128.3,128.0,127.7,127.5,127.4$, $126.8,126.2,125.7,124.5,121.7,121.5,116.5,37.7,32.7,24.4$.

HRMS (ESI-TOF) m/z: [M+H] $]^{+}$calcd for $\mathrm{C}_{38} \mathrm{H}_{31} \mathrm{~N}_{2} \mathrm{O}_{3}$ 563.2329; Found 563.2337<smiles>CC(=O)O/C(P)=C(\CC(CC(=O)NO)c1ccccc1)c1ccccc1</smiles>

\section{(Z)-6-oxo-1,2-diphenyl-6-(quinolin-8-ylamino)hex-1-en-1-yl acetate-d3 (40)}

$36.7 \mathrm{mg}, 81 \%$ yield, sticky yellow liquid.

$\boldsymbol{R}_{\boldsymbol{f}}: 0.32$ (hexane/ethyl acetate, $8: 2 \mathrm{v} / \mathrm{v}$ ).

IR (CHCl $): v 3365,3048,3021,2931,2844,2436,1953,1874,1756,1682,1647,1581,1536$, $1474,1421,1370,1217,1059,930,577 \mathrm{~cm}^{-1}$.

${ }^{1}$ H NMR (400 MHz, CDCl $) \delta 9.69(\mathrm{~s}, 1 \mathrm{H}), 8.78(\mathrm{dd}, J=4.2,1.5,1 \mathrm{H}), 8.71(\mathrm{dd}, J=7.0,1.7$, $1 \mathrm{H}), 8.14(\mathrm{dd}, J=8.3,1.5,1 \mathrm{H}), 7.55-7.46(\mathrm{~m}, 4 \mathrm{H}), 7.43(\mathrm{dd}, J=8.3,4.2,1 \mathrm{H}), 7.39-7.32$ (m, 4H), $7.33-7.23(\mathrm{~m}, 4 \mathrm{H}), 2.67-2.55(\mathrm{~m}, 2 \mathrm{H}), 2.48(\mathrm{t}, J=7.6,2 \mathrm{H}), 1.88(\mathrm{dt}, J=15.3,7.7$, 2H).

${ }^{13}$ C NMR (100 MHz, $\left.\mathbf{C D C l}_{3}\right) \delta 171.1,169.6,148.1,143.2,138.4,138.3,136.4,135.5,134.5$, $130.6,129.0,128.5,128.4,128.3,128.1,127.9,127.4,127.3,121.6,121.4,116.4,37.6,32.4$, 24.4.

HRMS (ESI-TOF) m/z: [M+H] $]^{+}$calcd for $\mathrm{C}_{29} \mathrm{H}_{24} \mathrm{~N}_{2} \mathrm{D}_{3} \mathrm{O}_{3} 454.2204$; Found 454.2202.<smiles>CC(=O)Oc1ccccc1C(=O)O/C(P)=C(\CC(CC(=O)NO)c1ccccc1)c1ccccc1</smiles>

(Z)-6-ox0-1,2-diphenyl-6-(quinolin-8-ylamino)hex-1-en-1-yl 2-acetoxybenzoate (4p)

$37.1 \mathrm{mg}, 65 \%$ yield, white solid, m.p $=198-200{ }^{\circ} \mathrm{C}$.

$\boldsymbol{R}_{\boldsymbol{f}}: 0.23$ (hexane/ethyl acetate, $8: 2 \mathrm{v} / \mathrm{v}$ ).

IR (solid): v 3479, 3035, 2966, 2931, 2400, 1890, 1749, 1614, 1425, 1304, 1156, 1066, 907, $826 \mathrm{~cm}^{-1}$. 
${ }^{1}$ H NMR (400 MHz, $\left.\mathbf{C D C l}_{3}\right) \delta 9.70(\mathrm{~s}, 1 \mathrm{H}), 8.79(\mathrm{dd}, J=4.2,1.5,1 \mathrm{H}), 8.71(\mathrm{~d}, J=7.0,1 \mathrm{H})$, $8.16(\mathrm{dd}, J=8.3,1.4,1 \mathrm{H}), 7.63(\mathrm{dd}, J=7.8,1.4,1 \mathrm{H}), 7.57-7.42(\mathrm{~m}, 6 \mathrm{H}), 7.37-7.25(\mathrm{~m}$, $7 \mathrm{H}), 7.24-7.12(\mathrm{~m}, 2 \mathrm{H}), 6.99(\mathrm{~d}, J=8.0,1 \mathrm{H}), 2.72-2.61(\mathrm{~m}, 2 \mathrm{H}), 2.51(\mathrm{t}, J=7.6,2 \mathrm{H}), 2.12$ $(\mathrm{s}, J=11.2,3 \mathrm{H}), 1.98-1.86(\mathrm{~m}, 2 \mathrm{H})$.

${ }^{13}$ C NMR (100 MHz, $\left.\mathbf{C D C l}_{3}\right) \delta 171.2,169.6,163.2,150.7,148.2,143.1,138.4,138.2,136.5$, $135.3,134.6,133.9,131.8,131.2,129.0,128.6,128.5,128.4,128.1,128.1,127.6,127.5,126.0$, $123.9,123.2,121.7,121.5,116.6,37.8,32.7,24.6,20.9$.

HRMS (ESI-TOF) m/z: [M+Na $]^{+}$calcd for $\mathrm{C}_{36} \mathrm{H}_{30} \mathrm{~N}_{2} \mathrm{NaO}_{5}$ 593.2047; Found 593.2020.<smiles>CC(C)NCC(=O)O/C(P)=C(\CC(CC(=O)NO)c1ccccc1)c1ccccc1</smiles>

\section{(Z)-6-oxo-1,2-diphenyl-6-(quinolin-8-ylamino)hex-1-en-1-yl acetylglycinate (4q)}

$31.0 \mathrm{mg}, 61 \%$ yield, yellow liquid.

$\boldsymbol{R}_{\boldsymbol{f}}: 0.10$ (hexane/ethyl acetate, $8: 2 \mathrm{v} / \mathrm{v}$ ).

IR (CHCl $\mathbf{3})$ : $\mathbf{~ 3 4 5 2 , ~ 3 3 7 2 , ~ 3 0 7 7 , ~ 3 0 7 0 , ~ 3 0 2 5 , ~ 2 9 6 0 , ~ 2 8 7 0 , ~ 2 6 7 0 , ~ 2 4 4 0 , ~ 2 4 3 3 , ~ 1 9 5 3 , ~ 1 8 8 0 , ~ 1 7 6 3 , ~}$ 1747, 1740, 1683, 1653, 1591, 1531, 1457, 1424, 1376, 1323, 1217, 1055, 930, 823, $573 \mathrm{~cm}^{-1}$. ${ }^{1} \mathbf{H}$ NMR (500 MHz, CDCl $) \delta 9.69(\mathrm{~s}, 1 \mathrm{H}), 8.80(\mathrm{~d}, J=3.5,1 \mathrm{H}), 8.71(\mathrm{~d}, J=6.7,1 \mathrm{H}), 8.18$ $(\mathrm{d}, J=8.2,1 \mathrm{H}), 7.55-7.45(\mathrm{~m}, 5 \mathrm{H}), 7.39$ (t, $J=7.5,2 \mathrm{H}), 7.34-7.26(\mathrm{~m}, 6 \mathrm{H}), 3.80(\mathrm{~d}, J=$ $4.9,2 \mathrm{H}), 2.68-2.58(\mathrm{~m}, 2 \mathrm{H}), 2.49(\mathrm{t}, J=7.5,2 \mathrm{H}), 1.94(\mathrm{~s}, 3 \mathrm{H}), 1.88(\mathrm{dt}, J=15.3,7.5,2 \mathrm{H})$.

${ }^{13}$ C NMR (125 MHz, $\left.\mathbf{C D C l}_{3}\right) \delta 171.1,170.0,168.8,148.2,142.9,138.4,137.9,136.5,134.8$, $134.5,131.3,129.0,128.8,128.6,128.5,128.0,127.6,127.5,121.7,121.5,116.5,41.4,37.5$, 32.4, 24.3, 23.0.

HRMS (ESI-TOF) m/z: [M+H] $]^{+}$calcd for $\mathrm{C}_{31} \mathrm{H}_{30} \mathrm{~N}_{3} \mathrm{O}_{4}$ 508.2231; Found 508.2233.<smiles>CCC(C)C([PH2+])C(=O)O/C(=C(\CCC(=O)NO)c1ccccc1)c1ccccc1</smiles>

(Z)-6-oxo-1,2-diphenyl-6-(quinolin-8-ylamino)hex-1-en-1-yl (2S)-2-(1,3-dioxoisoindolin2-yl)-3-methylpentanoate (4r)

$38.5 \mathrm{mg}, 59 \%$ yield, sticky liquid.

$\boldsymbol{R}_{\boldsymbol{f}}: 0.15$ (hexane/ethyl acetate, $8: 3 \mathrm{v} / \mathrm{v}$ ). 
IR (CHCl $\mathbf{3}):$ v 3402,3063, 3030, 3001, 2950, 2880, 2680, 2410, 2423, 1943, 1878, 1753, 1717 , $1710,1680,1651,1581,1541,1480,1447,1434,1386,1366,1333,1227,1045,1020,933$, $824,572 \mathrm{~cm}^{-1}$.

${ }^{1}$ H NMR (400 MHz, CDCl $)$ ) $\delta 9.64(\mathrm{~s}, 1 \mathrm{H}), 8.77(\mathrm{dd}, J=4.2,1.6,1 \mathrm{H}), 8.66(\mathrm{dd}, J=6.6,2.3$, $1 \mathrm{H}), 8.14(\mathrm{dd}, J=8.3,1.6,1 \mathrm{H}), 7.77(\mathrm{dt}, J=7.0,3.5,2 \mathrm{H}), 7.74-7.69(\mathrm{~m}, 2 \mathrm{H}), 7.53-7.38(\mathrm{~m}$, $5 \mathrm{H}), 7.26-7.20(\mathrm{~m}, 3 \mathrm{H}), 7.18(\mathrm{~d}, J=7.0,2 \mathrm{H}), 7.13(\mathrm{t}, J=7.6,2 \mathrm{H}), 6.99(\mathrm{t}, J=7.2,1 \mathrm{H}), 4.31$ $(\mathrm{d}, J=9.7,1 \mathrm{H}), 2.56(\mathrm{dd}, J=8.8,6.0,2 \mathrm{H}), 2.51-2.39(\mathrm{~m}, 3 \mathrm{H}), 1.80(\mathrm{dt}, J=15.2,7.6,2 \mathrm{H})$, $1.26-1.18(\mathrm{~m}, 1 \mathrm{H}), 0.93-0.82(\mathrm{~m}, 2 \mathrm{H}), 0.76(\mathrm{~d}, J=6.7,3 \mathrm{H}), 0.71(\mathrm{t}, J=7.4,3 \mathrm{H})$.

${ }^{13}$ C NMR (100 MHz, CDCl 3$) \delta 171.1,167.3,167.0,148.2,143.0,138.4,138.0,136.5,134.8$, $134.5,134.1,131.8,131.1,129.1,128.6,128.3,128.0,127.5,127.12,123.6,121.7,121.5$, $116.5,57.6,37.6,33.2,32.6,25.5,24.2,16.3,10.6$.

HRMS (ESI-TOF) m/z: [M+Na] $]^{+}$calcdfor $\mathrm{C}_{41} \mathrm{H}_{37} \mathrm{~N}_{3} \mathrm{NaO}_{5}$ 674.2625; Found 674.2621.

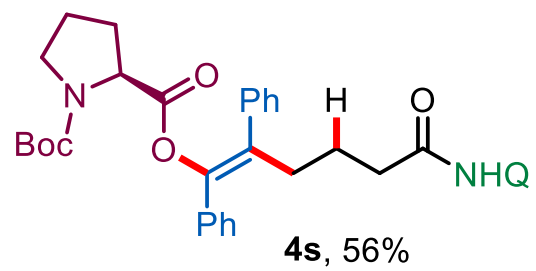

(Z)-1-(tert-butyl) 2-(6-0xo-1,2-diphenyl-6-(quinolin-8-ylamino)hex-1-en-1-yl) pyrrolidine-1,2-dicarboxylate (4s)

$33.9 \mathrm{mg}, 56 \%$ yield, liquid.

$\boldsymbol{R}_{\boldsymbol{f}}: 0.17$ (hexane/ethyl acetate, $8: 3 \mathrm{v} / \mathrm{v}$ ).

IR (CHCl $\mathbf{3})$ : v 3407, 3047, 3021, 2915, 2860, 2567, 2535, 2435, 1961, 1871, 1765, 1740, 1735, $1687,1680,1630,1591,1529,1477,1446,1376,1330,1227,1107,1077,1035,930,824,560$ $\mathrm{cm}^{-1}$.

${ }^{1}$ H NMR (500 MHz, CDCl $)$ ) $\delta 9.69(\mathrm{~s}, J=7.2,1 \mathrm{H}), 8.81(\mathrm{dd}, J=4.1,1.4,1 \mathrm{H}), 8.70$ (dd, $J=$ $6.9,1.8,1 \mathrm{H}), 8.18(\mathrm{dd}, J=8.2,1.3,1 \mathrm{H}), 7.56-7.49(\mathrm{~m}, 4 \mathrm{H}), 7.47(\mathrm{dd}, J=8.2,4.2,1 \mathrm{H}), 7.38$ (t, $J=7.5,2 \mathrm{H}), 7.33-7.22(\mathrm{~m}, 6 \mathrm{H}), 4.08(\mathrm{dd}, J=8.9,2.8,1 \mathrm{H}), 3.44-3.34(\mathrm{~m}, 1 \mathrm{H}), 3.30$ $3.21(\mathrm{~m}, 1 \mathrm{H}), 2.57-2.51(\mathrm{~m}, 2 \mathrm{H}), 2.50-2.45(\mathrm{~m}, 2 \mathrm{H}), 1.91-1.82(\mathrm{~m}, 2 \mathrm{H}), 1.81-1.72(\mathrm{~m}$, 1H), $1.60-1.51(\mathrm{~m}, 1 \mathrm{H}), 1.38-1.30(\mathrm{~m}, 1 \mathrm{H}), 1.13(\mathrm{~s}, 9 \mathrm{H}), 1.08-0.99(\mathrm{~m}, 1 \mathrm{H})$.

${ }^{13}$ C NMR (125 MHz, $\left.\mathbf{C D C l}_{3}\right) \delta 171.1,171.0,154.0,148.2,143.3,138.4,138.3,136.5,135.1$, $134.6,131.4,129.6,129.0,128.6,128.5,128.4,128.3,128.2,128.0,127.5,127.4,121.7,121.5$, $116.6,79.9,58.9,46.2,37.6,33.0,28.5,28.1,24.0,23.1$.

HRMS (ESI-TOF) m/z: [M+H] $]^{+}$calcd for $\mathrm{C}_{37} \mathrm{H}_{40} \mathrm{~N}_{3} \mathrm{O}_{5}$ 606.2962; Found 606.2965. 


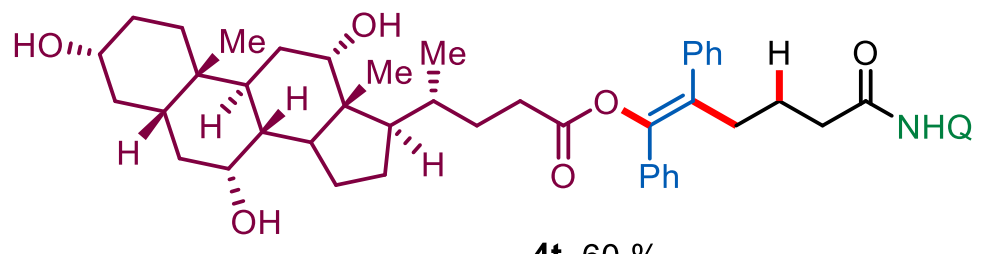

4t, $60 \%$

(Z)-6-oxo-1,2-diphenyl-6-(quinolin-8-ylamino)hex-1-en-1-yl

(4R)-4-

((3R,5S,7R,8R,9S,10S,12S,13R,17R)-3,7,12-trihydroxy-10,13-dimethylhexadecahydro-

\section{H-cyclopenta[a]phenanthren-17-yl)pentanoate (4t)}

$47.9 \mathrm{mg}, 60 \%$ yield, yellow liquid.

$\boldsymbol{R}_{\boldsymbol{f}}: 0.20$ (hexane/ethyl acetate, $8: 4 \mathrm{v} / \mathrm{v}$ ).

IR (CHCl $\mathbf{3})$ : $\mathbf{~ 3 5 0 3 , ~ 3 4 8 0 , ~ 3 4 6 0 , ~ 3 4 1 0 , 3 0 5 1 , ~ 3 0 3 2 , ~ 3 0 1 1 , ~ 2 9 4 1 , ~ 2 8 7 9 , ~ 2 6 7 5 , ~ 2 4 1 5 , ~ 2 4 3 1 , ~} 1937$, $1871,1751,1740,1677,1641,1577,1555,1475,1444,1420,1380,1360,1327,1237,1117$, $1090,1050,1011,944,860,824,667,620,560 \mathrm{~cm}^{-1}$.

${ }^{1}$ H NMR (400 MHz, $\left.\mathbf{C D C l}_{3}\right) \delta 9.66(\mathrm{~s}, 1 \mathrm{H}), 8.77(\mathrm{dd}, J=4.2,1.7,1 \mathrm{H}), 8.68(\mathrm{dd}, J=6.9,2.0$, $1 \mathrm{H}), 8.13(\mathrm{dd}, J=8.3,1.6,1 \mathrm{H}), 7.52-7.45(\mathrm{~m}, 4 \mathrm{H}), 7.43$ (dd, $J=8.3,4.2,1 \mathrm{H}), 7.35-7.28$ (m, 4H), $7.27-7.22(\mathrm{~m}, 4 \mathrm{H}), 3.93-3.74(\mathrm{~m}, 2 \mathrm{H}), 3.43-3.36(\mathrm{~m}, 1 \mathrm{H}), 2.77(\mathrm{~s}, 3 \mathrm{H}), 2.62-$ $2.54(\mathrm{~m}, 2 \mathrm{H}), 2.46(\mathrm{t}, J=7.6,2 \mathrm{H}), 2.23-2.06(\mathrm{~m}, 3 \mathrm{H}), 2.00-1.80(\mathrm{~m}, 5 \mathrm{H}), 1.75-1.68(\mathrm{~m}$, $3 \mathrm{H}), 1.66-1.58(\mathrm{~m}, 3 \mathrm{H}), 1.49(\mathrm{dd}, J=13.0,11.1,4 \mathrm{H}), 1.41-1.31(\mathrm{~m}, 2 \mathrm{H}), 1.17-1.07$ (m, 2H), $1.08-0.90(\mathrm{~m}, 4 \mathrm{H}), 0.85(\mathrm{~s}, J=9.2,4 \mathrm{H}), 0.78(\mathrm{~d}, J=6.4,3 \mathrm{H}), 0.56(\mathrm{~s}, 3 \mathrm{H})$.

${ }^{13}$ C NMR (100 MHz, CDCl $) \delta 172.7,171.2,148.2,143.3,138.4,138.4,136.4,135.6,134.5$, 130.6, 129.0, 128.4, 128.3, 128.3, 128.2, 128.0, 127.5, 127.3, 121.7, 121.4, 116.5, 73.1, 72.0, $68.6,47.1,46.5,41.7,41.5,39.6,37.6,35.0,34.8,34.7,32.5,31.1,30.5,28.2,27.4,26.4,24.4$, $23.3,22.5,17.2,12.6$.

HRMS (ESI-TOF) m/z: [M+H] $]^{+}$calcd for $\mathrm{C}_{51} \mathrm{H}_{63} \mathrm{~N}_{2} \mathrm{O}_{6}$ 799.4681; Found 799.4668.<smiles>COc1ccc(/C(CC(CC(N)=O)CC(C)(C)C)=C(\OC(C)=O)c2ccc(OC)cc2)cc1</smiles>

(Z)-1,2-bis(4-methoxyphenyl)-6-0xo-6-(quinolin-8-ylamino)hex-1-en-1-yl acetate (5a) 
$43.9 \mathrm{mg}, 86 \%$ yield, yellow liquid.

$\boldsymbol{R}_{\boldsymbol{f}}: 0.20$ (hexane/ethyl acetate, $8: 2 \mathrm{v} / \mathrm{v}$ ).

IR (CHCl $)$ : v 3410, 3046, 3001, 2938, 2862, 2652, 2447, 2410, 1961, 1879, 1768, 17381688 , $1646,1567,1537,1456,1424,1396,1335,1280,1109,1073,937,829,551 \mathrm{~cm}^{-1}$.

${ }^{1}$ H NMR (500 MHz, CDCl $) \delta 9.69(\mathrm{~s}, 1 \mathrm{H}), 8.77(\mathrm{dd}, J=44.0,5.0,2 \mathrm{H}), 8.17(\mathrm{~d}, J=8.1,1 \mathrm{H})$, $7.57-7.44(\mathrm{~m}, 3 \mathrm{H}), 7.45-7.37(\mathrm{~m}, 2 \mathrm{H}), 7.30(\mathrm{dd}, J=25.8,8.2,2 \mathrm{H}), 6.92-6.72(\mathrm{~m}, 4 \mathrm{H})$, $3.82(\mathrm{~s}, J=5.1,3 \mathrm{H}), 3.75(\mathrm{~s}, J=15.3,3 \mathrm{H}), 2.69-2.42(\mathrm{~m}, 4 \mathrm{H}), 2.02-1.65(\mathrm{~m}, 5 \mathrm{H})$.

${ }^{13}$ C NMR (125 MHz, $\left.\mathbf{C D C l}_{3}\right) \delta 171.3,169.6,159.5,158.7,148.2,142.9,138.4,136.5,134.6$, $133.1,130.7,130.3,130.0,129.5,129.3,128.2,128.0,127.5,121.7,121.5,116.5,114.1,113.8$, $113.7,55.3,37.6,32.5,24.5,20.9$.

HRMS (ESI-TOF) m/z: [M+Na $]^{+}$calcd for $\mathrm{C}_{31} \mathrm{H}_{30} \mathrm{~N}_{2} \mathrm{NaO}_{5}$ 533.2047; Found 533.2038.<smiles>CC(=O)O/C([15OH])=C(\CC(CC(=O)NO)c1ccc(F)cc1)c1ccc(F)cc1</smiles>

\section{(Z)-1,2-bis(4-fluorophenyl)-6-oxo-6-(quinolin-8-ylamino)hex-1-en-1-yl acetate (5b)}

$43.8 \mathrm{mg}, 90 \%$ yield, liquid.

$\boldsymbol{R}_{\boldsymbol{f}}: 0.29$ (hexane/ethyl acetate, 8:2 v/v).

IR (CHCl $\mathbf{3}):$ v 3417, 3067, 3025, 2960, 2845, 2690, 2445, 2413, 1960, 1884, 1767, 17391688 , $1647,1591,1556,1529,1477,1446,1378,1335,1227,1077,938,839,577 \mathrm{~cm}^{-1}$.

${ }^{1}$ H NMR (500 MHz, CDCl $) \delta 9.69(\mathrm{~s}, 1 \mathrm{H}), 8.84-8.78(\mathrm{~m}, 1 \mathrm{H}), 8.71(\mathrm{~d}, J=6.9,1 \mathrm{H}), 8.19$ $(\mathrm{d}, J=8.2,1 \mathrm{H}), 7.57-7.50(\mathrm{~m}, 2 \mathrm{H}), 7.50-7.44(\mathrm{~m}, 3 \mathrm{H}), 7.34-7.27(\mathrm{~m}, 2 \mathrm{H}), 7.07(\mathrm{t}, J=8.6$, $2 \mathrm{H}), 6.98(\mathrm{t}, J=8.6,2 \mathrm{H}), 2.59-2.53(\mathrm{~m}, 2 \mathrm{H}), 2.49(\mathrm{t}, J=7.4,2 \mathrm{H}), 2.01-1.75(\mathrm{~m}, 5 \mathrm{H})$.

${ }^{13}$ C NMR (125 MHz, CDCl3) $\delta$ 173.0, 169.5, 163.3 (d, $\left.J=285 \mathrm{~Hz}\right), 161.4$ (d, $J=280 \mathrm{~Hz}$ ), $148.2,142.7,138.4,136.5,134.2$ (d, $J=190 \mathrm{~Hz}), 131.5,131.0,130.9,130.1,129.8$ (d, $J=30$ $\mathrm{Hz}), 128.0,127.5,121.7$ (d, $J=70 \mathrm{~Hz}), 116.5,115.5,115.4$ (d, $J=85 \mathrm{~Hz}), 37.4,32.5,24.3$, 20.8 .

HRMS (ESI-TOF) m/z: [M+Na] $]^{+}$calcd for $\mathrm{C}_{29} \mathrm{H}_{24} \mathrm{~N}_{2} \mathrm{NaF}_{2} \mathrm{O}_{3}$ 509.1647; Found 509.1649. 


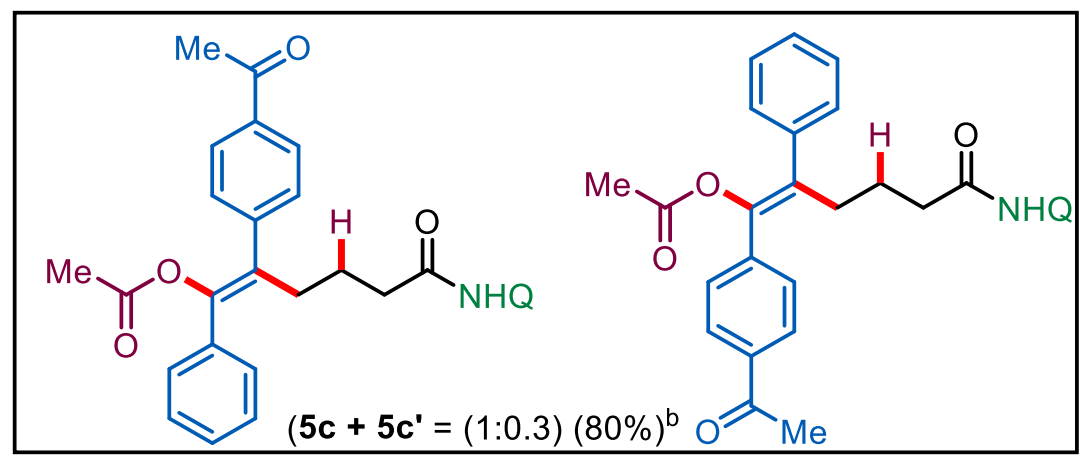

(Z)-2-(4-acetylphenyl)-6-oxo-1-phenyl-6-(quinolin-8-ylamino)hex-1-en-1-yl acetate(5c)

$39.4 \mathrm{mg}, 80 \%$ yield, brown sticky liquid.

$\boldsymbol{R}_{\boldsymbol{f}}: 0.32$ (hexane/ethyl acetate, $8: 2 \mathrm{v} / \mathrm{v}$ ).

IR (CHCl $)$ : v 3398, 3066, 3010, 2930, 2860, 2662, 2441, 2428, 1948, 1883, 1777, 1740, 1717 , $1687,1645,1570,1530,1490,1441,1377,1337,1216,1070,930,847,590 \mathrm{~cm}^{-1}$.

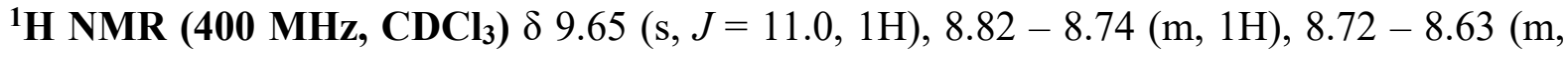
1H), $8.21-8.09(\mathrm{~m}, 1 \mathrm{H}), 8.01-7.77(\mathrm{~m}, 2 \mathrm{H}), 7.59-7.43(\mathrm{~m}, 6 \mathrm{H}), 7.40-7.23(\mathrm{~m}, 4 \mathrm{H}), 2.66$ $-2.59(\mathrm{~m}, 2 \mathrm{H}), 2.58-2.49(\mathrm{~m}, 3 \mathrm{H}), 2.49-2.43(\mathrm{~m}, 2 \mathrm{H}), 1.91-1.82(\mathrm{~m}, 2 \mathrm{H}), 1.82(\mathrm{~s}, 3 \mathrm{H})$.

${ }^{13}$ C NMR (100 MHz, $\left.\mathbf{C D C l}_{3}\right) \delta 197.9,171.0,169.4,148.2,144.0,143.8,138.3,136.5,136.0$, $135.2,134.4,129.8,129.1,128.9,128.8,128.5,128.4,128.4,128.0,127.5,121.7,121.6,116.6$, $37.4,32.2,26.7,24.4,20.8$.

HRMS (ESI-TOF) m/z: [M+Na $]^{+}$calcd for $\mathrm{C}_{31} \mathrm{H}_{28} \mathrm{~N}_{2} \mathrm{NaO}_{4}$ 515.1941; Found 515.1963.<smiles>CC(=O)OC(=C(CCC(=O)NO)COC1(C)C=CC(=O)C=C1)c1ccccc1</smiles>

(Z)-6-oxo-6-(quinolin-8-ylamino)-1-(thiophen-2-yl)-2-((p-tolyloxy)methyl)hex-1-en-1-yl acetate $(5 d)$

$45.9 \mathrm{mg}, 90 \%$ yield, liquid.

$\boldsymbol{R}_{\boldsymbol{f}}: 0.19$ (hexane/ethyl acetate, $8: 2 \mathrm{v} / \mathrm{v}$ ).

IR (CHCl $\mathbf{3})$ : v 3403, 3030, 3026, 2938, 2842, 2670, 2440, 2420, 1949, 1876, 1760, 1737, 1716, $1684,1654,1578,1567,1477,1436,1377,1344,1297,1227,1045,955,847,597 \mathrm{~cm}^{-1}$. 


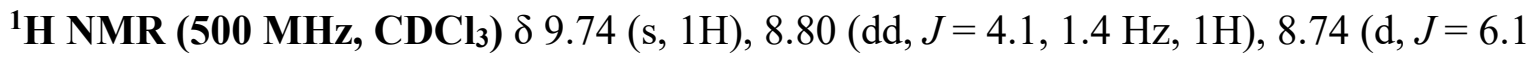
$\mathrm{Hz}, 1 \mathrm{H}), 8.17(\mathrm{dd}, J=8.2,1.3 \mathrm{~Hz}, 1 \mathrm{H}), 7.55-7.48$ (m, 2H), 7.46 (dd, $J=8.2,4.2 \mathrm{~Hz}, 1 \mathrm{H})$, $7.35(\mathrm{dd}, J=11.0,4.7 \mathrm{~Hz}, 2 \mathrm{H}), 7.29-7.23(\mathrm{~m}, 3 \mathrm{H}), 6.89$ (d, $J=10.1 \mathrm{~Hz}, 2 \mathrm{H}), 6.33$ (d, $J=$ $10.1 \mathrm{~Hz}, 2 \mathrm{H}), 4.01(\mathrm{~s}, 2 \mathrm{H}), 2.51(\mathrm{t}, J=7.4 \mathrm{~Hz}, 2 \mathrm{H}), 2.41-2.34(\mathrm{~m}, 2 \mathrm{H}), 2.07(\mathrm{~s}, 3 \mathrm{H}), 2.04-$ $1.96(\mathrm{~m}, 2 \mathrm{H}), 1.46(\mathrm{~s}, 3 \mathrm{H})$.

${ }^{13}$ C NMR (125 MHz, CDCl $)$ ) $185.4,171.3,168.9,152.0,148.2,144.9,138.3,136.5,135.0$, $134.5,130.2,128.8,128.6,128.3,128.0,127.5,126.1,121.7,121.5,116.5,72.8,62.0,37.6$, $28.5,26.4,24.2,20.8$.

HRMS (ESI-TOF) m/z: [M+Na $]^{+}$calcd for $\mathrm{C}_{31} \mathrm{H}_{30} \mathrm{~N}_{2} \mathrm{NaO}_{5}$ 533.2047; Found 533.2052.<smiles>CC(=O)O/C(=C(\CCC(N)=O)COC1(C)C=CC(=O)C=C1)c1ccc(Br)cc1</smiles>

(Z)-1-(4-bromophenyl)-2-(((1-methyl-4-oxocyclohexa-2,5-dien-1-yl)oxy)methyl)-6-oxo-6(quinolin-8-ylamino)hex-1-en-1-yl acetate (5e)

$50.0 \mathrm{mg}, 85 \%$ yield, liquid.

$\boldsymbol{R}_{\boldsymbol{f}}: 0.22$ (hexane/ethyl acetate, $8: 2 \mathrm{v} / \mathrm{v}$ )

IR (CHCl $):$ v 3410, 3040, 3030, 2928, 2862, 2680, 2470, 2445, 1957, 1888, 1762, 1739, 1715, $1688,1657,1655,1580,1550,1466,1439,1368,1338,1289,1231,1067,990,862,580 \mathrm{~cm}^{-1}$. ${ }^{1}$ H NMR (400 MHz, CDCl $) \delta 9.70(\mathrm{~s}, 1 \mathrm{H}), 8.78(\mathrm{dd}, J=4.1,1.5 \mathrm{~Hz}, 1 \mathrm{H}), 8.70(\mathrm{dd}, J=7.1$, $1.5 \mathrm{~Hz}, 1 \mathrm{H}), 8.16(\mathrm{dd}, J=8.3,1.4 \mathrm{~Hz}, 1 \mathrm{H}), 7.56-7.48(\mathrm{~m}, 2 \mathrm{H}), 7.45(\mathrm{dd}, J=8.2,4.2 \mathrm{~Hz}, 1 \mathrm{H})$, $7.33(\mathrm{t}, J=7.6 \mathrm{~Hz}, 2 \mathrm{H}), 7.19(\mathrm{~d}, J=8.4 \mathrm{~Hz}, 2 \mathrm{H}), 6.85(\mathrm{~d}, J=10.2 \mathrm{~Hz}, 2 \mathrm{H}), 6.31(\mathrm{~d}, J=10.1$ $\mathrm{Hz}, 2 \mathrm{H}), 3.96$ (s, 2H), 2.49 (t, $J=7.3 \mathrm{~Hz}, 2 \mathrm{H}), 2.35-2.29$ (m, 2H), 2.04 (s, 3H), $1.99-1.93$ (m, 2H), 1.43 (s, $J=9.4 \mathrm{~Hz}, 3 \mathrm{H})$.

${ }^{13}$ C NMR (100 MHz, CDC13) $\delta$ 185.3, 171.1, 168.9, 151.9, 148.2, 143.8, 138.3, 136.6, 134.4, $133.9,131.5,130.5,130.3,128.0,127.5,126.9,122.8,121.7,121.6,116.6,72.8,61.9,37.4$, $28.4,26.4,24.1,20.8$. 
HRMS (ESI-TOF) m/z: [M+Na $]^{+}$calcd for $\mathrm{C}_{31} \mathrm{H}_{29} \mathrm{BrN}_{2} \mathrm{NaO}_{5}$ 611.1152; Found 611.1158.<smiles>CC(=O)O/C(=C(\CC=CCC(=O)NO)COC1(c2ccccc2)C=CC(=O)C=C1)c1ccccc1</smiles>

(Z)-6-0xo-2-(((4-0xo-[1,1'-biphenyl]-1(4H)-yl)oxy)methyl)-1-phenyl-6-(quinolin-8ylamino)hex-1-en-1-yl acetate $(5 \mathrm{f})$

$44.7 \mathrm{mg}, 78 \%$ yield, yellow liquid.

$\boldsymbol{R}_{\boldsymbol{f}}: 0.26$ (hexane/ethyl acetate, $8: 2 \mathrm{v} / \mathrm{v}$ ).

IR (CHCl $\mathbf{3})$ : v 3403, 3030, 3026, 2938, 2842, 2670, 2440, 2420, 1949, 1876, 1760, 1737, 1716, $1684,1654,1578,1567,1477,1436,1377,1344,1297,1227,1045,955,847,597 \mathrm{~cm}^{-1}$.

${ }^{1} \mathbf{H}$ NMR (500 MHz, CDCl$)_{3} \delta 9.75(\mathrm{~s}, 1 \mathrm{H}), 8.78(\mathrm{~d}, J=2.8,1 \mathrm{H}), 8.76-8.70(\mathrm{~m}, 1 \mathrm{H}), 8.18$ $(\mathrm{d}, J=8.1,1 \mathrm{H}), 7.57-7.43(\mathrm{~m}, 5 \mathrm{H}), 7.39$ (d, $J=6.5,2 \mathrm{H}), 7.29(\mathrm{tt}, J=14.5,7.4,6 \mathrm{H}), 6.93(\mathrm{~d}$, $J=10.0,2 \mathrm{H}), 6.44(\mathrm{~d}, J=10.0,2 \mathrm{H}), 4.28(\mathrm{~s}, 2 \mathrm{H}), 2.56(\mathrm{t}, J=7.4,2 \mathrm{H}), 2.54-2.45(\mathrm{~m}, 2 \mathrm{H})$, $2.16-2.04(\mathrm{~m}, 5 \mathrm{H})$.

${ }^{13}$ C NMR (125 MHz, CDCl$\left._{3}\right) \delta 185.7,171.1,168.9,150.7,148.2,145.1,138.4,138.2,136.5$, $135.0,134.5,129.9,128.9,128.9,128.7,128.4,128.3,128.0,127.5,126.0,125.9,121.7,121.5$, $116.6,76.6,61.5,37.6,28.6,24.2,20.9$.

HRMS (ESI-TOF) m/z: [M+Na $]^{+}$calcd for $\mathrm{C}_{36} \mathrm{H}_{32} \mathrm{~N}_{2} \mathrm{NaO}_{5}$ 595.2203; Found 595.2201.<smiles>CCCCCCCCCCCC(=O)NO</smiles>

(Z)-6-0xo-1-phenyl-2-(phenylethynyl)-6-(quinolin-8-ylamino)hex-1-en-1-yl acetate (5g)

$31.3 \mathrm{mg}, 68 \%$ yield,lightyellow liquid.

$\boldsymbol{R}_{\boldsymbol{f}}: 0.30$ (hexane/ethyl acetate, 8:2 v/v) 
IR (CHCl $):$ v 3409, 3096, 3033, 2939, 2863, 2705, 2670, 2444, 2417, 1965, 1879, 1844, 1768, 1745, 1739, 1689, 1637, 1610, 1594, 1567, 1536, 1476, 1436, 1373, 1333, 1213, 1045, 925, $826,561,465 \mathrm{~cm}^{-1}$.

${ }^{1}$ H NMR (400 MHz, CDCl3) $\delta 9.77(\mathrm{~s}, 1 \mathrm{H}), 8.74(\mathrm{dd}, J=4.2,1.6,1 \mathrm{H}), 8.70(\mathrm{dd}, J=6.8$, $2.1,1 \mathrm{H}), 8.15(\mathrm{dd}, J=8.3,1.6,1 \mathrm{H}), 7.52-7.48(\mathrm{~m}, 2 \mathrm{H}), 7.48-7.41(\mathrm{~m}, 3 \mathrm{H}), 7.41-7.37$ $(\mathrm{m}, 2 \mathrm{H}), 7.32-7.28(\mathrm{~m}, 3 \mathrm{H}), 7.25-7.21(\mathrm{~m}, 3 \mathrm{H}), 2.62(\mathrm{t}, J=7.5,2 \mathrm{H}), 2.55(\mathrm{t}, J=7.3,2 \mathrm{H})$, $2.25(\mathrm{~s}, 3 \mathrm{H}), 2.25-2.18(\mathrm{~m}, 2 \mathrm{H})$.

${ }^{13}$ C NMR (100 MHz, $\left.\mathbf{C D C l}_{3}\right) \delta 171.3,168.9,151.8,148.2,138.4,136.4,134.6,134.2,131.7$, 129.0, 128.7, 128.5, 128.4, 128.3, 128.0, 127.5, 121.6, 121.5, 116.6, 113.7, 96.5, 85.9, 37.0, $30.3,24.6,21.0$.

HRMS (ESI-TOF) m/z: [M+Na] $]^{+}$calcd for $\mathrm{C}_{31} \mathrm{H}_{26} \mathrm{~N}_{2} \mathrm{NaO}_{3}$ 497.1836; Found 497.1839.<smiles>CC(=O)O/C(=C(/C#Cc1ccc(C)cc1)CC(CC(=O)NO)c1ccc(C)cc1)c1ccccc1</smiles>

(Z)-6-oxo-6-(quinolin-8-ylamino)-1-(p-tolyl)-2-(p-tolylethynyl)hex-1-en-1-yl acetate (5h) $33.7 \mathrm{mg}, 67 \%$ yield, light yellow liquid.

$\boldsymbol{R}_{\boldsymbol{f}}: 0.34$ (hexane/ethyl acetate, $8: 2 \mathrm{v} / \mathrm{v}$ )

IR (CHCl $)$ : v 3410, 3091, 3043, 2943, 2852, 2710, 2668, 2439, 2411, 1968, 1878, 1854, 1765, 1740, 1736, 1687, 1629, 1621, 1584, 1562, 1531, 1478, 1455, 1371, 1347, 1215, 1048, 921, $830,569,470 \mathrm{~cm}^{-1}$.

${ }^{1}$ H NMR (500 MHz, CDCl 3 ) $\delta 9.77(\mathrm{~s}, 1 \mathrm{H}), 8.78(\mathrm{dd}, J=4.2,1.5 \mathrm{~Hz}, 1 \mathrm{H}), 8.71(\mathrm{dd}, J=7.0$, $1.7 \mathrm{~Hz}, 1 \mathrm{H}), 8.18$ (dd, $J=8.2,1.4 \mathrm{~Hz}, 1 \mathrm{H}), 7.53-7.50(\mathrm{~m}, 1 \mathrm{H}), 7.47$ (dd, $J=8.2,4.2 \mathrm{~Hz}$, 1H), $7.37(\mathrm{~d}, J=8.0 \mathrm{~Hz}, 2 \mathrm{H}), 7.31-7.25(\mathrm{~m}, 3 \mathrm{H}), 7.12(\mathrm{~d}, J=7.9 \mathrm{~Hz}, 2 \mathrm{H}), 7.02(\mathrm{~d}, J=7.9$ $\mathrm{Hz}, 2 \mathrm{H}), 2.64$ (t, $J=7.4 \mathrm{~Hz}, 2 \mathrm{H}), 2.57$ (t, $J=7.2 \mathrm{~Hz}, 2 \mathrm{H}), 2.37$ (s, 3H), 2.26 (s, 3H), 2.25 (s, $3 \mathrm{H}), 2.24-2.19(\mathrm{~m}, 2 \mathrm{H})$. 
${ }^{13}$ C NMR (125 MHz, CDCl 3$) \delta 171.4,168.9,151.7,148.1,139.0,138.6,136.4,134.6,131.6$, $131.4,129.8,129.2$, 129.0, 128.5, 128.0, 127.5, 121.6, 121.4, 120.3, 116.7, 113.3, 96.5, 85.4, $36.9,30.3,24.6,21.6,21.4,21.1$.

HRMS (ESI-TOF) m/z: [M+Na $]^{+}$calcd for $\mathrm{C}_{33} \mathrm{H}_{30} \mathrm{~N}_{2} \mathrm{NaO}_{3}$ 525.2149; Found 525.2150.<smiles>CC(=O)O/C(=C(/C#Cc1cccs1)CCC(=O)NO)c1cccs1</smiles>

(Z)-6-oxo-6-(quinolin-8-ylamino)-1-(thiophen-2-yl)-2-(thiophen-2-ylethynyl)hex-1-en-1yl acetate (5i)

$29.2 \mathrm{mg}, 60 \%$ yield, brown liquid.

$\boldsymbol{R}_{\boldsymbol{f}}: 0.30$ (hexane/ethyl acetate, $8: 2 \mathrm{v} / \mathrm{v}$ )

IR (CHCl $\mathbf{3})$ : v 3415, 3082, 3053, 2937, 2854, 2727, 2657, 2443, 2427, 1963, 1876, 1851, 1770, $1742,1738,1686,1632,1623,1577,1562,1520,1480,1462,1373,1290,1060,890,669,560$ $\mathrm{cm}^{-1}$.

${ }^{1}$ H NMR (400 MHz, $\left.\mathbf{C D C l}_{3}\right) \delta 9.79(\mathrm{~s}, 1 \mathrm{H}), 8.79-8.70(\mathrm{~m}, 2 \mathrm{H}), 8.16(\mathrm{dd}, J=8.3,1.5,1 \mathrm{H})$, $7.55-7.48(\mathrm{~m}, 2 \mathrm{H}), 7.47-7.42(\mathrm{~m}, 2 \mathrm{H}), 7.40(\mathrm{dd}, J=2.9,1.2,1 \mathrm{H}), 7.26-7.24(\mathrm{~m}, 1 \mathrm{H}), 7.17$ $(\mathrm{dd}, J=5.0,3.0,1 \mathrm{H}), 7.12(\mathrm{ddd}, J=8.4,5.0,1.2,2 \mathrm{H}), 2.66(\mathrm{t}, J=7.4,2 \mathrm{H}), 2.64-2.59$ (m, $2 \mathrm{H}), 2.26(\mathrm{~s}, J=4.8,3 \mathrm{H}), 2.25-2.19(\mathrm{~m}, 2 \mathrm{H})$.

${ }^{13}$ C NMR (100 MHz, $\left.\mathbf{C D C l}_{3}\right) \delta 171.4,168.9,148.2,147.3,138.4,136.5,135.0,134.6,129.9$, $128.8,128.1,127.6,127.3,125.8,125.7,125.5,122.4,121.7,121.5,116.7,113.5,91.8,85.7$, 37.0, 30.4, 24.5, 21.0 .

HRMS (ESI-TOF) m/z: [M+H $]^{+}$calcd for $\mathrm{C}_{27} \mathrm{H}_{23} \mathrm{~N}_{2} \mathrm{~S}_{2} \mathrm{O}_{3} 487.1145$; Found 487.1150.<smiles>CC(=O)O/C(=C(\Br)CCC(=O)NO)c1ccccc1</smiles>

(Z)-2-bromo-6-oxo-1-phenyl-6-(quinolin-8-ylamino)hex-1-en-1-yl acetate (5j) $35.3 \mathrm{mg}, 78 \%$ yield, brown sticky liquid. 
$\boldsymbol{R}_{\boldsymbol{f}}: 0.19$ (hexane/ethyl acetate, $8: 2 \mathrm{v} / \mathrm{v}$ )

IR (CHCl $):$ v 3367, 3108, 3076, 3058, 3017, 2937, 2845, 2667, 2470, 2407, 1961, 1886, 1773, $1727,1689,1657,1586,1532,1479,1443,1325,1227,1080,987,810,687,526 \mathrm{~cm}^{-1}$.

${ }^{1}$ H NMR (500 MHz, CDCl $) \delta 9.77(\mathrm{~s}, 1 \mathrm{H}), 8.84(\mathrm{dd}, J=4.0,1.2,1 \mathrm{H}), 8.69$ (dd, $J=6.1,2.6$, $1 \mathrm{H}), 8.20(\mathrm{~d}, J=7.6,1 \mathrm{H}), 7.56-7.51(\mathrm{~m}, 2 \mathrm{H}), 7.49(\mathrm{dd}, J=8.3,4.0,1 \mathrm{H}), 7.41-7.35(\mathrm{~m}, 2 \mathrm{H})$, $7.24-7.18(\mathrm{~m}, 3 \mathrm{H}), 2.71(\mathrm{t}, J=7.2,2 \mathrm{H}), 2.57(\mathrm{t}, J=7.4,2 \mathrm{H}), 2.22(\mathrm{~s}, 3 \mathrm{H}), 2.20-2.14(\mathrm{~m}$, $2 \mathrm{H})$.

${ }^{13}$ C NMR (125 MHz, $\left.\mathbf{C D C l}_{3}\right) \delta 170.9,168.2,148.2,145.6,136.6,134.4,133.8,130.3,129.1$, 128.8, 128.6, 128.5, 128.0, 127.6, 121.7, 121.6, 119.0, 116.8, 36.2, 34.7, 24.4, 20.9.

HRMS (ESI-TOF) m/z: [M+Na] $]^{+}$calcd for $\mathrm{C}_{23} \mathrm{H}_{21} \mathrm{BrN}_{2} \mathrm{NaO}_{3} 475.0628$; Found 475.0621 .<smiles>CCCCC(=O)OC(C)=O</smiles>

\section{(E)-10-0xo-10-(quinolin-8-ylamino)dec-5-en-5-yl acetate (5k)}

$32.6 \mathrm{mg}, 92 \%$ yield, liquid.

$\boldsymbol{R}_{\boldsymbol{f}}: 0.32$ (hexane/ethyl acetate, $8: 2 \mathrm{v} / \mathrm{v}$ )

IR (CHCl $):$ v 3408, 3080, 3027, 2921, 2867, 2810, 2673, 2437, 2410, 1966, 1886, 1780, 1762 , $1740,1685,1644,1598,1530,1490,1466,1340,1215,1065,930,840,577 \mathrm{~cm}^{-1}$.

${ }^{1}$ H NMR (400 MHz, CDCl $) \delta 9.81(\mathrm{~s}, 1 \mathrm{H}), 8.78(\mathrm{dd}, J=4.1,1.5,1 \mathrm{H}), 8.75(\mathrm{dd}, J=7.2,1.4$, $1 \mathrm{H}), 8.13(\mathrm{dd}, J=8.3,1.5,1 \mathrm{H}), 7.55-7.45(\mathrm{~m}, 2 \mathrm{H}), 7.42(\mathrm{dd}, J=8.3,4.2,1 \mathrm{H}), 5.13(\mathrm{t}, J=$ $7.7,1 \mathrm{H}), 2.59(\mathrm{t}, J=7.5,2 \mathrm{H}), 2.25-2.12(\mathrm{~m}, 4 \mathrm{H}), 2.11(\mathrm{~s}, 3 \mathrm{H}), 1.97-1.83(\mathrm{~m}, 2 \mathrm{H}), 1.41-$ $1.31(\mathrm{~m}, 2 \mathrm{H}), 1.30-1.21(\mathrm{~m}, 2 \mathrm{H}), 0.82(\mathrm{t}, J=7.2,3 \mathrm{H})$.

${ }^{13}$ C NMR (100 MHz, $\left.\mathbf{C D C l}_{3}\right) \delta 171.6,169.9,149.8,148.2,138.4,136.5,134.5,128.0,127.5$, 121.7, 121.6, 117.0, 116.6, 37.2, 29.0, 28.8, 25.9, 25.5, 22.3, 21.1, 13.9.

HRMS (ESI-TOF) m/z: [M+Na $]^{+}$calcd for $\mathrm{C}_{21} \mathrm{H}_{26} \mathrm{~N}_{2} \mathrm{NaO}_{3} 377.1836$; Found 377.1829.<smiles>CCCCCCCCCC(=O)NO</smiles>

(E)-1-0xo-1-(quinolin-8-ylamino)undec-5-en-6-yl acetate (5l) 
$32.4 \mathrm{mg}, 88 \%$ yield, liquid.

$\boldsymbol{R}_{\boldsymbol{f}}: 0.36$ (hexane/ethyl acetate, $8: 2 \mathrm{v} / \mathrm{v}$ )

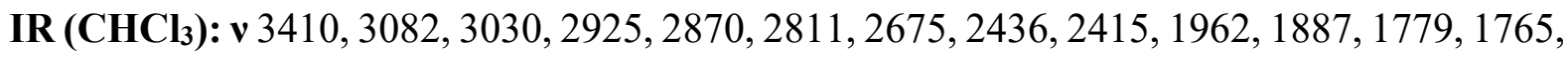
$1738,1681,1645,1591,1531,1489,1467,1342,1220,1066,940,820,560 \mathrm{~cm}^{-1}$.

${ }^{1}$ H NMR (400 MHz, CDCl $) \delta 9.80(\mathrm{~s}, 1 \mathrm{H}), 8.76(\mathrm{dd}, J=9.7,2.6 \mathrm{~Hz}, 2 \mathrm{H}), 8.13(\mathrm{~d}, J=8.2$ $\mathrm{Hz}, 1 \mathrm{H}), 7.54-7.45$ (m, 2H), 7.42 (dd, $J=8.2,4.2 \mathrm{~Hz}, 1 \mathrm{H}), 5.13$ (t, $J=7.7 \mathrm{~Hz}, 1 \mathrm{H}), 2.59$ (t, $J=7.5 \mathrm{~Hz}, 2 \mathrm{H}), 2.21(\mathrm{dt}, J=14.4,7.4 \mathrm{~Hz}, 4 \mathrm{H}), 2.11(\mathrm{~s}, 3 \mathrm{H}), 1.95-1.85(\mathrm{~m}, 2 \mathrm{H}), 1.42-1.32$ (m, 2H), $1.25-1.17(\mathrm{~m}, 4 \mathrm{H}), 0.81(\mathrm{t}, J=6.7 \mathrm{~Hz}, 3 \mathrm{H})$.

${ }^{13}$ C NMR (100 MHz, CDCl 3$) \delta 171.5,169.8,149.8,148.2,138.4,136.4,134.5,128.0,127.5$, 121.6, 121.5, 117.0, 116.6, 37.2, 31.4, 29.0, 26.6, 25.9, 25.5, 22.5, 21.1, 14.0.

HRMS (ESI-TOF) m/z: [M+Na] $]^{+}$calcd for $\mathrm{C}_{22} \mathrm{H}_{28} \mathrm{~N}_{2} \mathrm{NaO}_{3}$ 391.1992; Found 391.2006.<smiles>CC(=O)O/C(=C/CCCC(=O)NO)c1ccccc1</smiles>

\section{(E)-6-0xo-1-phenyl-6-(quinolin-8-ylamino)hex-1-en-1-yl acetate (5m)}

$27.3 \mathrm{mg}, 76 \%$ yield, light yellow liquid.

$\boldsymbol{R}_{\boldsymbol{f}}: 0.30$ (hexane/ethyl acetate, $8: 2 \mathrm{v} / \mathrm{v}$ ).

IR (CHCl $):$ v 3380, 3080, 3026, 2927, 2860, 2670, 2443, 2427, 1953, 1883, 1781, 1740, 1830, $1683,1655,1588,1537,1473,1431,1396,1330,1230,1080,935,886,685 \mathrm{~cm}^{-1}$.

${ }^{1}$ H NMR (400 MHz, CDCl 3$) \delta 9.76(\mathrm{~s}, 1 \mathrm{H}), 8.80(\mathrm{dd}, J=4.1,1.5,1 \mathrm{H}), 8.73(\mathrm{dd}, J=6.9,1.8$, $1 \mathrm{H}), 8.16(\mathrm{dd}, J=8.3,1.4,1 \mathrm{H}), 7.55-7.48(\mathrm{~m}, 2 \mathrm{H}), 7.45$ (dd, $J=8.3,4.2,1 \mathrm{H}), 7.36$ (dd, $J=7.8$, $1.5,2 \mathrm{H}), 7.29-7.23(\mathrm{~m}, 3 \mathrm{H}), 5.50(\mathrm{t}, J=7.7,1 \mathrm{H}), 2.56(\mathrm{t}, J=7.6,2 \mathrm{H}), 2.37(\mathrm{q}, J=7.5,2 \mathrm{H}), 2.14$ (s, 3H), $2.01-1.91(\mathrm{~m}, 2 \mathrm{H})$.

${ }^{13}$ C NMR (100 MHz, CDC13) $\delta$ 171.4, 169.8, 148.2, 147.1, 138.4, 136.6, 134.5, 134.4, 128.5, $128.3,128.3,128.1,127.6,121.7,121.6,119.5,116.7,37.4,26.7,25.7,21.1$.

HRMS (ESI-TOF) m/z: [M+Na] $]^{+}$calcd for $\mathrm{C}_{23} \mathrm{H}_{22} \mathrm{~N}_{2} \mathrm{NaO}_{3} 397.1523$; Found 397.1524. 
<smiles>CC(=O)CC(CC(N)=O)C/C(=C\OC(C)=O)COc1ccc(C)cc1</smiles>

5 n, $37 \%$

\section{(E)-7-ox0-7-(quinolin-8-ylamino)-1-(p-tolyloxy)hept-2-en-2-yl acetate (5n)}

$15.5 \mathrm{mg}, 37 \%$ yield, yellow liquid.

$\boldsymbol{R}_{f}: 0.23$ (hexane/ethyl acetate, $8: 2 \mathrm{v} / \mathrm{v}$ )

IR (CHCl $\mathbf{3})$ : v 3380, 3100, 3070, 3010, 2985, 2860, 2767, 2660, 2400, 1941, 1883, 1777, 1740, $1690,1657,1580,1532,1479,1423,1390,1340,1236,1163,1077,989,867,687,581,487$ $\mathrm{cm}^{-1}$.

${ }^{1}$ H NMR (400 MHz, CDCl $)$ ) $\delta 9.81(\mathrm{~s}, 1 \mathrm{H}), 8.85-8.67(\mathrm{~m}, 2 \mathrm{H}), 8.16(\mathrm{dd}, J=8.3,1.6,1 \mathrm{H})$, $7.59-7.48(\mathrm{~m}, 2 \mathrm{H}), 7.45(\mathrm{dd}, J=8.3,4.2,1 \mathrm{H}), 7.19(\mathrm{~s}, 1 \mathrm{H}), 7.11-6.94(\mathrm{~m}, 2 \mathrm{H}), 6.92-6.69$ $(\mathrm{m}, 2 \mathrm{H}), 4.68(\mathrm{~s}, J=8.4,2 \mathrm{H}), 2.64-2.54(\mathrm{~m}, 2 \mathrm{H}), 2.34-2.22(\mathrm{~m}, 5 \mathrm{H}), 2.13(\mathrm{~s}, J=3.5,3 \mathrm{H})$, $2.08-1.99(\mathrm{~m}, 2 \mathrm{H})$.

${ }^{13}$ C NMR (100 MHz, CDCl $) \delta 171.5,167.6,156.6,148.2,138.4,136.6,134.6,133.3,130.3$, 130.0, 128.1, 127.6, 121.7, 121.6, 120.4, 116.7, 114.9, 114.7, 63.1, 37.6, 29.4, 23.7, 20.8, 20.6. HRMS (ESI-TOF) m/z: [M+Na $]^{+}$calcd for $\mathrm{C}_{25} \mathrm{H}_{26} \mathrm{~N}_{2} \mathrm{NaO}_{4} 441.1785$; Found 441.1781.<smiles>CC(=O)O/C(=C/CCCC(=O)NO)COc1ccc(C)cc1</smiles>

\section{(Z)-6-0x0-6-(quinolin-8-ylamino)-2-((p-tolyloxy)methyl)hex-1-en-1-yl acetate (5n')}

$10.9 \mathrm{mg}, 28 \%$ yield, yellow liquid.

$\boldsymbol{R}_{\boldsymbol{f}}: 0.22$ (hexane/ethyl acetate, $8: 2 \mathrm{v} / \mathrm{v}$ )

IR (CHCl $\mathbf{3})$ : v 3382, 3105, 3073, 3015, 2970, 2855, 2770, 2657, 2405, 1950, 1881, 1772, 1741, $1689,1660,1577,1530,1480,1425,1389,1339,1232,1159,1088,987,863,677,591,497$ $\mathrm{cm}^{-1}$. 
${ }^{1}$ H NMR (400 MHz, CDCl $) \delta 9.81(\mathrm{~s}, 1 \mathrm{H}), 8.93-8.58(\mathrm{~m}, 2 \mathrm{H}), 8.16(\mathrm{dd}, J=8.3,1.6,1 \mathrm{H})$, $7.64-7.39(\mathrm{~m}, 3 \mathrm{H}), 7.10-6.93(\mathrm{~m}, 2 \mathrm{H}), 6.88-6.71(\mathrm{~m}, 2 \mathrm{H}), 5.49(\mathrm{t}, J=8.0,1 \mathrm{H}), 4.61(\mathrm{~s}$, $2 \mathrm{H}), 2.65-2.55(\mathrm{~m}, 2 \mathrm{H}), 2.36-2.27(\mathrm{~m}, 2 \mathrm{H}), 2.23(\mathrm{~s}, J=13.2,3 \mathrm{H}), 2.14(\mathrm{~s}, J=3.4,3 \mathrm{H}), 1.99$ $-1.88(\mathrm{~m}, 2 \mathrm{H})$.

${ }^{13}$ C NMR (100 MHz, $\left.\mathbf{C D C l}_{3}\right) \delta 171.3,170.0,156.4,148.2,144.8,136.6,134.5,130.6,130.0$, 128.1, 127.6, 122.6, 121.7, 121.6, 116.7, 114.9, 114.7, 64.0, 36.9, 25.8, 25.1, 21.0, 20.6.

HRMS (ESI-TOF) m/z: [M+Na] $]^{+}$calcd for $\mathrm{C}_{25} \mathrm{H}_{26} \mathrm{~N}_{2} \mathrm{NaO}_{4} 441.1784$; Found 441.1781.<smiles>CC(=O)O/C(=C/CC(/C=C/CC(=O)NO)CC(=O)NO)CCC(=O)NO</smiles>

(5Z,12E)-1,17-dioxo-1,17-bis(quinolin-8-ylamino)heptadeca-5,12-diene-6,12-diyl diacetate (50)

$53.2 \mathrm{mg}, 80 \%$ yield, light yellow liquid.

$\boldsymbol{R}_{\boldsymbol{f}}: 0.16$ (hexane/ethyl acetate, $8: 2 \mathrm{v} / \mathrm{v}$ )

IR (CHCl $\mathbf{3})$ : v 3382, 3070, 3050, 2980, 2892, 2697, 2470, 2412, 1967, 1910, 1881, 1768, 1738, $1681,1670,1598,1527,1487,1449,1381,1330,1290,1170,1095,935,890,690,610,581$, $530,470 \mathrm{~cm}^{-1}$.

${ }^{1}$ H NMR (500 MHz, CDCl $) \delta 9.82(\mathrm{~s}, 1 \mathrm{H}), 8.80(\mathrm{dd}, J=11.7,5.7,2 \mathrm{H}), 8.16(\mathrm{~d}, J=8.2,1 \mathrm{H})$, $7.53(\mathrm{dt}, J=16.4,8.0,2 \mathrm{H}), 7.46(\mathrm{dd}, J=8.2,4.2,1 \mathrm{H}), 5.15(\mathrm{t}, J=7.6,1 \mathrm{H}), 2.61(\mathrm{t}, J=7.4$, 2H), $2.27-2.16(\mathrm{~m}, 4 \mathrm{H}), 2.13(\mathrm{~s}, J=4.7,3 \mathrm{H}), 1.92(\mathrm{dt}, J=14.7,7.4,2 \mathrm{H}), 1.39-1.34(\mathrm{~m}$, $2 \mathrm{H}), 1.27-1.23(\mathrm{~m}, 1 \mathrm{H}), 0.95-0.86(\mathrm{~m}, 1 \mathrm{H})$.

${ }^{13}$ C NMR (125 MHz, CDCl 3$) \delta 171.5,169.8,149.6,148.3,138.4,136.5,134.6,128.1,127.5$, $121.7,121.5,117.2,116.6,37.2,29.0,28.9,26.7,26.0,25.5,21.2$.

HRMS (ESI-TOF) m/z: [M+Na $]^{+}$calcd for $\mathrm{C}_{39} \mathrm{H}_{44} \mathrm{~N}_{4} \mathrm{NaO}_{6} 687.3153$; Found 687.3174. 
<smiles>CCCC/C(=C\C(C)CCC(=O)NO)OC(C)=O</smiles>

$5 p, 72 \%$

\section{(R,E)-7-methyl-10-oxo-10-(quinolin-8-ylamino)dec-5-en-5-yl acetate (5p)}

$26.5 \mathrm{mg}, 72 \%$ yield, liquid.

$\boldsymbol{R}_{\boldsymbol{f}}: 0.28$ (hexane/ethyl acetate, $8: 2 \mathrm{v} / \mathrm{v}$ )

IR ( $\left.\mathbf{C H C l}_{\mathbf{3}}\right)$ : v 3367, 3045, 3030, 2990, 2860, 2690, 2445, 2410, 1963, 1881, 1767, 1745, 1690, $1645,1570,1531,1491,1455,1370,1330,1232,1070,990,930,831,561 \mathrm{~cm}^{-1}$.

${ }^{1}$ H NMR (500 MHz, CDCl 3$) \delta 9.83(\mathrm{~s}, 1 \mathrm{H}), 8.83(\mathrm{dd}, J=4.1,1.5 \mathrm{~Hz}, 1 \mathrm{H}), 8.80$ (d, $J=7.4 \mathrm{~Hz}$, $1 \mathrm{H}), 8.18(\mathrm{dd}, J=8.2,1.4 \mathrm{~Hz}, 1 \mathrm{H}), 7.53(\mathrm{dt}, J=8.1,7.5 \mathrm{~Hz}, 2 \mathrm{H}), 7.47(\mathrm{dd}, J=8.2,4.2 \mathrm{~Hz}$, $1 \mathrm{H}), 4.84(\mathrm{~d}, J=10.7 \mathrm{~Hz}, 1 \mathrm{H}), 2.70-2.66(\mathrm{~m}, 1 \mathrm{H}), 2.63-2.53(\mathrm{~m}, 1 \mathrm{H}), 2.29-2.20(\mathrm{~m}, 3 \mathrm{H})$, $2.15(\mathrm{~s}, 3 \mathrm{H}), 1.37-1.30(\mathrm{~m}, 4 \mathrm{H}), 1.24-1.20(\mathrm{~m}, 2 \mathrm{H}), 0.98-0.93(\mathrm{~m}, 5 \mathrm{H}), 0.78(\mathrm{t}, J=7.3 \mathrm{~Hz}$, $3 \mathrm{H})$.

${ }^{13}$ C NMR (125 MHz, CDCl $) \delta 171.9,169.8,149.0,148.2,138.5,136.4,134.7,128.1,127.5$, $123.2,121.7,121.5,116.6,35.9,33.1,31.5,29.2,29.1,22.4,21.8,21.2,13.9$.

HRMS (ESI-TOF) m/z: [M+Na $]^{+}$calcd for $\mathrm{C}_{22} \mathrm{H}_{28} \mathrm{~N}_{2} \mathrm{NaO}_{3}$ 391.1992; Found 391.2005.<smiles>C#CC(/C=C(\CCCC)OC(C)=O)CC(=O)NO</smiles>

$5 q, 58 \%$

\section{(E)-7-ethyl-10-oxo-10-(quinolin-8-ylamino)dec-5-en-5-yl acetate (5q)}

$22.2 \mathrm{mg}, 58 \%$ yield, liquid.

$\boldsymbol{R}_{\boldsymbol{f}}: 0.29$ (hexane/ethyl acetate, $8: 2 \mathrm{v} / \mathrm{v}$ )

IR (CHCl) $)$ v 3377, 3037, 3024, 2978, 2847, 2685, 2457, 2431, 1959, 1873, 1771, 1737, 1688, $1655,1581,1526,1487,1461,1366,1334,1246,1073,980,943,833,590,490 \mathrm{~cm}^{-1}$.

${ }^{1}$ H NMR (400 MHz, $\left.\mathbf{C D C l}_{3}\right) \delta 9.81(\mathrm{~s}, 1 \mathrm{H}), 8.87-8.68(\mathrm{~m}, 2 \mathrm{H}), 8.15(\mathrm{dd}, J=8.3,1.6,1 \mathrm{H})$, $7.58-7.47$ (m, 2H), 7.44 (dd, $J=8.3,4.2,1 \mathrm{H}), 4.82$ (d, $J=10.7,1 \mathrm{H}), 2.66$ (ddd, $J=14.4,8.8$, $5.4,1 \mathrm{H}), 2.60-2.50(\mathrm{~m}, 1 \mathrm{H}), 2.26-2.17(\mathrm{~m}, 2 \mathrm{H}), 2.13(\mathrm{~s}, 3 \mathrm{H}), 1.85(\mathrm{td}, J=6.6,3.0,1 \mathrm{H})$, $1.62-1.49(\mathrm{~m}, 4 \mathrm{H}), 1.21-1.02(\mathrm{~m}, 4 \mathrm{H}), 0.97-0.92(\mathrm{~m}, 4 \mathrm{H}), 0.75(\mathrm{t}, J=7.3,3 \mathrm{H})$. 
${ }^{13}$ C NMR (125 MHz, CDCl 3$) \delta 172.0,169.9,150.0,148.2,138.5,136.5,134.7,128.1,127.6$, $121.9,121.7,121.5,116.6,38.6,35.8,31.3,29.2,29.2,29.1,22.5,21.2,13.9,12.0$.

HRMS (ESI-TOF) m/z: [M+H] $]^{+}$calcd for $\mathrm{C}_{23} \mathrm{H}_{31} \mathrm{~N}_{2} \mathrm{O}_{3} 383.2329$; Found 383.2332.<smiles>CCCCCC(OC(C)=O)C(=O)CCCC(=O)NO</smiles>

$7 a, 80 \%$

\section{7,12-dioxo-12-(quinolin-8-ylamino)dodecan-6-yl acetate (7a)}

$31.9 \mathrm{mg}, 80 \%$ yield, light brown liquid.

$\boldsymbol{R}_{\boldsymbol{f}}: 0.20$ (hexane/ethyl acetate, $8: 2 \mathrm{v} / \mathrm{v}$ )

IR (CHCl $)$ : v3405, 3105, 3080, 2950, 2860, 2670, 2449, 2437, 1961, 1880, 1810, 1763, 1741, $1725,1692,1655,1588,1535,1491,1430,1377,1336,1226,1046,929,827,690,577,490$ $\mathrm{cm}^{-1}$.

${ }^{1}$ H NMR (500 MHz, CDCl $) \delta 9.83$ (s, 1H), 8.83 (d, $\left.J=3.1,1 \mathrm{H}\right), 8.79$ (d, $\left.J=7.1,1 \mathrm{H}\right), 8.18$ $(\mathrm{d}, J=7.7,1 \mathrm{H}), 7.59-7.50(\mathrm{~m}, 2 \mathrm{H}), 7.48(\mathrm{dd}, J=8.2,4.2,1 \mathrm{H}), 5.01(\mathrm{dd}, J=8.4,4.3,1 \mathrm{H})$, $2.66-2.47(\mathrm{~m}, 4 \mathrm{H}), 2.16(\mathrm{~s}, 3 \mathrm{H}), 1.84(\mathrm{dd}, J=14.9,7.5,2 \mathrm{H}), 1.79-1.69(\mathrm{~m}, 5 \mathrm{H}), 1.42-1.37$ (m, 2H), $1.33-1.28(\mathrm{~m}, 4 \mathrm{H}), 0.90(\mathrm{t}, J=6.5,3 \mathrm{H})$.

${ }^{13}$ C NMR (125 MHz, CDCl 3$) \delta 207.4,171.5,170.7,148.3,138.4,136.5,134.6,128.1,127.5$, 121.7, 121.6, 116.6, 78.7, 38.5, 38.0, 31.5, 30.5, 25.1, 25.1, 22.9, 22.5, 20.8, 14.1.

HRMS (ESI-TOF) m/z: [M+Na $]^{+}$calcd for $\mathrm{C}_{23} \mathrm{H}_{30} \mathrm{~N}_{2} \mathrm{NaO}_{4} 421.2098$; Found 421.2091.<smiles>CCCC(OC(C)=O)C(=O)CCCC(=O)NO</smiles>

\section{5,10-dioxo-10-(quinolin-8-ylamino)decan-4-yl acetate $(7 \mathrm{~b})$}

$28.9 \mathrm{mg}, 78 \%$ yield, liquid.

$\boldsymbol{R}_{\boldsymbol{f}}: 0.20$ (hexane/ethyl acetate, $8: 2 \mathrm{v} / \mathrm{v}$ ).

IR (CHCl $\mathbf{3}):$ v 3410, 3101, 3074, 2955, 2843, 2637, 2478, 2443, 1970, 1890, 1805, 1759, 1742, $1722,1688,1660,1589,1540,1487,1449,1387,1354,1234,1053,968,870,730,686,583$, $479 \mathrm{~cm}^{-1}$. 
${ }^{1}$ H NMR (500 MHz, CDCl $) \delta 9.84(\mathrm{~s}, 1 \mathrm{H}), 8.82(\mathrm{~d}, J=4.0,1 \mathrm{H}), 8.78(\mathrm{~d}, J=7.2,1 \mathrm{H}), 8.18$ $(\mathrm{d}, J=8.2,1 \mathrm{H}), 7.59-7.50(\mathrm{~m}, 2 \mathrm{H}), 7.48(\mathrm{dd}, J=8.2,4.2,1 \mathrm{H}), 5.17-4.86(\mathrm{~m}, 1 \mathrm{H}), 2.70-$ $2.44(\mathrm{~m}, 4 \mathrm{H}), 2.15(\mathrm{~s}, J=21.6,3 \mathrm{H}), 1.84(\mathrm{td}, J=14.6,7.2,2 \mathrm{H}), 1.80-1.66(\mathrm{~m}, 4 \mathrm{H}), 1.49-$ $1.38(\mathrm{~m}, 2 \mathrm{H}), 0.95(\mathrm{t}, J=7.4,3 \mathrm{H})$.

${ }^{13}$ C NMR (125 MHz, CDCl $\left.{ }_{3}\right) \delta$ 207.38, 171.49, 170.83, 148.22, 136.59, 134.54, 128.07, 127.6, $121.7,121.6,116.7,78.5,38.4,38.0,32.6,25.1,22.8,20.8,18.7,13.8$.

HRMS (ESI-TOF) m/z: [M+Na $]^{+}$calcd for $\mathrm{C}_{21} \mathrm{H}_{26} \mathrm{~N}_{2} \mathrm{NaO}_{4} 393.1785$; Found 393.1789.<smiles>CCCCC(CC)C(OC(C)=O)C(=O)CCCC(=O)NO</smiles>

\section{5-ethyl-7,12-dioxo-12-(quinolin-8-ylamino)dodecan-6-yl acetate (7c)}

$32.0 \mathrm{mg}, 75 \%$ yield, liquid.

$\boldsymbol{R}_{\boldsymbol{f}}: 0.22$ (hexane/ethyl acetate, $8: 2 \mathrm{v} / \mathrm{v}$ ).

IR (CHCl $\mathbf{3})$ : v 3390, 3099, 3074, 2967, 2852, 2634, 2480, 2439, 1968, 1888, 1810, 1764, 1741, 1723, 1685, 1667, 1591, 1537, 1490, 1451, 1392, 1348, 1239, 1049, 971, 868, 727, 689, 579, $481 \mathrm{~cm}^{-1}$.

${ }^{1}$ H NMR (500 MHz, CDCl $) \delta 9.83(\mathrm{~s}, 1 \mathrm{H}), 8.81(\mathrm{dd}, J=19.5,5.4,2 \mathrm{H}), 8.18(\mathrm{~d}, J=8.1,1 \mathrm{H})$, $7.68-7.37$ (m, 3H), $5.30-4.92(\mathrm{~m}, 1 \mathrm{H}), 2.68-2.39$ (m, 19H), $2.16(\mathrm{~s}, J=39.3,13 \mathrm{H}), 1.90-$ $1.82(\mathrm{~m}, 12 \mathrm{H}), 1.76(\mathrm{dt}, J=14.4,7.0,10 \mathrm{H}), 1.54-1.44(\mathrm{~m}, 7 \mathrm{H}), 1.39-1.19(\mathrm{~m}, 43 \mathrm{H}), 0.96-$ $0.87(\mathrm{~m}, 32 \mathrm{H})$.

${ }^{13}$ C NMR (125 MHz, $\left.\mathbf{C D C l}_{3}\right) \delta$ 207.6, 171.5, 170.9, 148.3, 138.4, 136.6, 134.6, 128.1, 127.6, $121.7,121.6,116.6,80.2$, 79.9, 41.3, 41.0, 39.1, 39.1, 38.0, 29.6, 29.6, 29.4, 29.0, 25.2 23.3, $23.0,22.9,22.4,20.8,14.1,11.9,11.8$.

HRMS (ESI-TOF) m/z: [M+Na $]^{+}$calcd for $\mathrm{C}_{25} \mathrm{H}_{34} \mathrm{~N}_{2} \mathrm{NaO}_{4} 449.2411$; Found 449.2415.<smiles>CC(=O)OC(C=CCC(=O)NO)c1ccccc1</smiles>

2,7-dioxo-1-phenyl-7-(quinolin-8-ylamino)heptyl acetate (7d)

$32.4 \mathrm{mg}, 80 \%$ yield, light brown liquid. 
$\boldsymbol{R}_{\boldsymbol{f}}: 0.21$ (hexane/ethyl acetate, $8: 2 \mathrm{v} / \mathrm{v}$ ).

IR (CHCl $):$ v 3413, 3110, 3080, 3005, 2970, 2860, 2633, 2480, 2441, 1966, 1890, 1805, 1771, $1738,1727,1690,1690,1655,1588,1565,1485,1445,1410,1397,1351,1241,1052,980$, $861,733,693,583,490,455 \mathrm{~cm}^{-1}$.

${ }^{1}$ H NMR (400 MHz, $\left.\mathbf{C D C l}_{3}\right) \delta 9.74(\mathrm{~s}, 1 \mathrm{H}), 8.79(\mathrm{dd}, J=4.2,1.6,1 \mathrm{H}), 8.73(\mathrm{dd}, J=7.0,1.8$, 1H), $8.14(\mathrm{dd}, J=8.3,1.6,1 \mathrm{H}), 7.55-7.46(\mathrm{~m}, 2 \mathrm{H}), 7.44(\mathrm{dd}, J=8.3,4.2,1 \mathrm{H}), 7.42-7.31$ $(\mathrm{m}, 5 \mathrm{H}), 5.97(\mathrm{~s}, 1 \mathrm{H}), 2.65-2.35(\mathrm{~m}, 4 \mathrm{H}), 2.18(\mathrm{~s}, J=6.4,3 \mathrm{H}), 1.77-1.60(\mathrm{~m}, 4 \mathrm{H})$.

${ }^{13}$ C NMR (100 MHz, $\left.\mathbf{C D C l}_{3}\right) \delta 203.8,171.4,170.5,148.2,138.4,136.5,134.5,133.2,129.5$, $129.2,128.2,128.0,127.5,121.7,121.5,116.5,80.8,38.4,37.7,24.8,22.9,20.8$.

HRMS (ESI-TOF) m/z: [M+Na $]^{+}$calcd for $\mathrm{C}_{24} \mathrm{H}_{24} \mathrm{~N}_{2} \mathrm{NaO}_{4} 427.1628$; Found 427.1630.<smiles>CC(=O)OC(C)CC(=O)CCCC(=O)NO</smiles>

\section{4,9-dioxo-9-(quinolin-8-ylamino)nonan-2-yl acetate (7e)}

$31.0 \mathrm{mg}, 87 \%$ yield, brown liquid.

$\boldsymbol{R}_{\boldsymbol{f}}: 0.19$ (hexane/ethyl acetate, $8: 2 \mathrm{v} / \mathrm{v}$ )

IR (CHCl $\mathbf{3}): \mathbf{v} 3401,3010,2970,2853,2734,2655,2490,2450,1973,1890,1812,1770,1733$, 1718, 1690, 1670, 1580, 1490, 1466, 1372, 1242, 1051, 968, 871, 731, 580, $483 \mathrm{~cm}^{-1}$.

${ }^{1}$ H NMR (500 MHz, CDCl $) \delta 9.82(\mathrm{~s}, 1 \mathrm{H}), 8.82(\mathrm{dd}, J=4.2,1.6,1 \mathrm{H}), 8.77(\mathrm{dd}, J=7.3,1.2$, $1 \mathrm{H}), 8.17(\mathrm{dd}, J=8.3,1.5,1 \mathrm{H}), 7.59-7.49(\mathrm{~m}, 2 \mathrm{H}), 7.47(\mathrm{dd}, J=8.2,4.2,1 \mathrm{H}), 5.47-5.16$ (m, 1H), $2.80(\mathrm{dd}, J=16.3,7.2,1 \mathrm{H}), 2.59(\mathrm{dd}, J=13.7,6.4,2 \mathrm{H}), 2.52(\mathrm{dd}, J=15.3,6.6,2 \mathrm{H})$, $2.01(\mathrm{~s}, 3 \mathrm{H}), 1.83(\mathrm{dq}, J=11.7,7.2,2 \mathrm{H}), 1.76-1.69(\mathrm{~m}, 2 \mathrm{H}), 1.27(\mathrm{~d}, J=6.3,3 \mathrm{H})$.

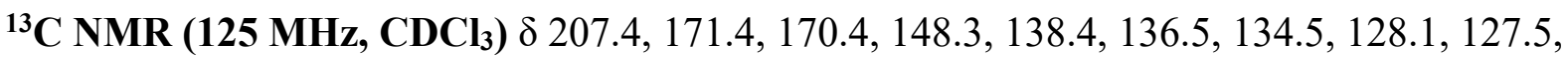
$121.7,121.6,116.6,67.2,48.6,43.1,37.9,25.1,23.2,21.3,20.2$.

HRMS (ESI-TOF) m/z: [M+Na] $]^{+}$calcd for $\mathrm{C}_{20} \mathrm{H}_{24} \mathrm{~N}_{2} \mathrm{NaO}_{4} 379.1628$; Found 379.1626.<smiles>CC(=O)OC(Br)CC(=O)CCCC(=O)NO</smiles>

7f, $83 \%$

2,2-dimethyl-5,10-dioxo-10-(quinolin-8-ylamino)decan-3-yl acetate (7f)

$33.1 \mathrm{mg}, 83 \%$ yield, brown liquid. 
$\boldsymbol{R}_{\boldsymbol{f}}: 0.20$ (hexane/ethyl acetate, $8: 2 \mathrm{v} / \mathrm{v}$ )

IR (CHCl $)$ : v 3401, 3088, 3010, 2980, 2860, 2640, 2479, 2441, 1973, 1879, 1807, 1770, 1739, $1716,1690,1655,1565,1510,1495,1465,1379,1352,1243,1057,969,877,721,690,582$, $488 \mathrm{~cm}^{-1}$.

${ }^{1}$ H NMR (400 MHz, CDCl $) \delta 9.78(\mathrm{~s}, 1 \mathrm{H}), 8.78(\mathrm{~d}, J=4.1,1 \mathrm{H}), 8.74(\mathrm{dd}, J=7.1,1.5,1 \mathrm{H})$, $8.13(\mathrm{~d}, J=8.2,1 \mathrm{H}), 7.54-7.45(\mathrm{~m}, 2 \mathrm{H}), 7.43(\mathrm{dd}, J=8.2,4.2,1 \mathrm{H}), 5.11(\mathrm{dd}, J=8.7,3.7$, 1H), $2.66-2.39$ (m, 6H), 1.98 (s, 3H), 1.78 (dd, $J=14.9,7.4,2 \mathrm{H}), 1.68$ (ddd, $J=19.6,12.6$, $7.1,2 \mathrm{H}), 0.88(\mathrm{~s}, 9 \mathrm{H})$.

${ }^{13}$ C NMR (100 MHz, $\left.\mathbf{C D C l}_{3}\right) \delta$ 208.2, 171.5, 170.6, 148.3, 138.4, 136.5, 134.6, 128.0, 127.5, $121.7,121.5,116.5,76.4,42.7,38.0,34.6,31.7,25.8,25.1,23.2,22.8$.

HRMS (ESI-TOF) m/z: [M+H] $]^{+}$calcd for $\mathrm{C}_{23} \mathrm{H}_{31} \mathrm{~N}_{2} \mathrm{O}_{4}$ 399.2278; Found 399.2282.<smiles>CC(=O)OC(CC(=O)CCCC(=O)NO)C1CCCCC1</smiles>

\section{1-cyclohexyl-3,8-dioxo-8-(quinolin-8-ylamino)octyl acetate (7g)}

$36.9 \mathrm{mg}, 87 \%$ yield, light yellow liquid.

$\boldsymbol{R}_{\boldsymbol{f}}: 0.25$ (hexane/ethyl acetate, $8: 2 \mathrm{v} / \mathrm{v}$ ).

IR (CHCl $\mathbf{3})$ : v 3389, 3065, 3020, 2990, 2870, 2643, 2430, 2410, 1980, 1880, 1810, 1765, 1737 , $1718,1688,1661,1556,1515,1456,1384,1357,1256,1047,980,869,715,680,590,479 \mathrm{~cm}^{-}$ 1 .

${ }^{1}$ H NMR (400 MHz, CDCl $) \delta 9.79(\mathrm{~s}, 1 \mathrm{H}), 8.78(\mathrm{dd}, J=4.2,1.6,1 \mathrm{H}), 8.74(\mathrm{dd}, J=7.1,1.8$, $1 \mathrm{H}), 8.14(\mathrm{dd}, J=8.3,1.6,1 \mathrm{H}), 7.54-7.46(\mathrm{~m}, 2 \mathrm{H}), 7.43(\mathrm{dd}, J=8.3,4.2,1 \mathrm{H}), 5.12(\mathrm{dt}, J=$ 8.0, 5.1, 1H), $2.69-2.41(\mathrm{~m}, 6 \mathrm{H}), 1.99(\mathrm{~s}, 3 \mathrm{H}), 1.83-1.75(\mathrm{~m}, 2 \mathrm{H}), 1.75-1.62(\mathrm{~m}, 7 \mathrm{H}), 1.55$ $-1.43(\mathrm{~m}, 1 \mathrm{H}), 1.24-1.10(\mathrm{~m}, 3 \mathrm{H}), 1.02-0.91(\mathrm{~m}, 2 \mathrm{H})$.

${ }^{13}$ C NMR (100 MHz, $\left.\mathbf{C D C l}_{3}\right) \delta 208.0,171.5,170.6,148.2,138.3,136.4,134.4,127.9,127.4$, 121.6, 121.5, 116.5, 73.7, 44.4, 42.9, 41.3, 37.8, 28.7, 28.1, 26.3, 26.0, 25.9, 25.0, 23.1, 21.1. HRMS (ESI-TOF) m/z: [M+H] $]^{+}$calcd for $\mathrm{C}_{25} \mathrm{H}_{33} \mathrm{~N}_{2} \mathrm{O}_{4} 425.2435$; Found 425.2441. 
<smiles>CC(CC(=O)CCCC(=O)NO)OC(=O)C1CCC1</smiles>

\section{4,9-dioxo-9-(quinolin-8-ylamino)nonan-2-yl cyclobutanecarboxylate (7h)}

$34.5 \mathrm{mg}, 87 \%$ yield, liquid.

$\boldsymbol{R}_{\boldsymbol{f}}: 0.20$ (hexane/ethyl acetate, $8: 2 \mathrm{v} / \mathrm{v}$ ).

IR (CHCl $\mathbf{3})$ : v 3409, 3070, 3010, 2987, 2867, 2657, 2441, 2422, 1970, 1878, 1808, 1761, 1735, $1720,1676,1663,1566,1523,1440,1369,1361,1237,1060,978,875,726,678,588,460 \mathrm{~cm}^{-}$ 1 .

${ }^{1}$ H NMR (400 MHz, CDCl $) \delta 9.80(\mathrm{~s}, 1 \mathrm{H}), 8.80(\mathrm{dd}, J=4.2,1.6,1 \mathrm{H}), 8.75(\mathrm{dd}, J=7.0,1.8$, $1 \mathrm{H}), 8.16(\mathrm{dd}, J=8.3,1.5,1 \mathrm{H}), 7.56-7.48(\mathrm{~m}, 2 \mathrm{H}), 7.45(\mathrm{dd}, J=8.3,4.2,1 \mathrm{H}), 5.49-4.95$ $(\mathrm{m}, 1 \mathrm{H}), 3.17$ (p, $J=8.5,1 \mathrm{H}), 3.05(\mathrm{p}, J=8.5,1 \mathrm{H}), 2.77(\mathrm{dd}, J=16.2,7.3,1 \mathrm{H}), 2.60-2.54$ $(\mathrm{m}, 2 \mathrm{H}), 2.53-2.47(\mathrm{~m}, 2 \mathrm{H}), 2.35-2.28(\mathrm{~m}, 2 \mathrm{H}), 2.25-2.19(\mathrm{~m}, 3 \mathrm{H}), 2.15-2.11(\mathrm{~m}, 1 \mathrm{H})$, $2.01-1.94(\mathrm{~m}, 1 \mathrm{H}), 1.93-1.88(\mathrm{~m}, 1 \mathrm{H}), 1.83-1.78(\mathrm{~m}, 1 \mathrm{H}), 1.73-1.67(\mathrm{~m}, 1 \mathrm{H}), 1.24(\mathrm{~d}, J$ $=6.3,3 \mathrm{H})$.

${ }^{13}$ C NMR (100 MHz, CDCl $) \delta$ 207.6, 174.7, 171.6, 148.3, 138.4, 136.6, 134.5, 128.1, 127.5, $121.7,121.7,116.7,67.0,48.7,43.2,38.3,38.0,25.3,25.2,23.2,20.2,18.5,18.4$.

HRMS (ESI-TOF) m/z: [M+Na $]^{+}$calcd for $\mathrm{C}_{23} \mathrm{H}_{28} \mathrm{~N}_{2} \mathrm{NaO}_{4} 419.1941$; Found 419.1948.<smiles>C=C(CC)C(=O)CCC(=O)NO</smiles>

$7 \mathrm{i}, 77 \%$

\section{5-methylene-6-oxo-N-(quinolin-8-yl)octanamide (7i)}

$22.8 \mathrm{mg}, 77 \%$ yield, yellow liquid.

$\boldsymbol{R}_{\boldsymbol{f}}: 0.23$ (hexane/ethyl acetate, $8: 2 \mathrm{v} / \mathrm{v}$ ).

IR (CHCl $\mathbf{3}):$ v 3392, 3106, 3080, 2985, 2890, 2650, 2466, 2425, 1985, 1860, 1710, 1690, 1666, $1570,1523,1461,1355,1330,1270,1064,970,872,732,593,460 \mathrm{~cm}^{-1}$.

${ }^{1}$ H NMR (400 MHz, CDCl $) \delta 9.80(\mathrm{~s}, J=42.3,1 \mathrm{H}), 8.80(\mathrm{dd}, J=4.2,1.6,1 \mathrm{H}), 8.77$ (dd, $J=$ $7.2,1.5,1 \mathrm{H}), 8.15(\mathrm{dd}, J=8.3,1.5,1 \mathrm{H}), 7.63-7.32(\mathrm{~m}, 3 \mathrm{H}), 6.04(\mathrm{~s}, 1 \mathrm{H}), 5.82(\mathrm{~s}, 1 \mathrm{H}), 2.72$ $(\mathrm{q}, J=7.3,2 \mathrm{H}), 2.58(\mathrm{t}, J=7.5,2 \mathrm{H}), 2.49-2.35(\mathrm{~m}, 2 \mathrm{H}), 2.02-1.87(\mathrm{~m}, 2 \mathrm{H}), 1.09(\mathrm{t}, J=7.3$, $3 \mathrm{H})$. 
${ }^{13}$ C NMR (100 MHz, CDCl 3$) \delta 202.5,171.5,148.3,147.9,138.4,136.5,134.6,128.1,127.5$, $124.4,121.7,121.5,116.5,37.7,31.0,30.5,24.5,8.6$.

HRMS (ESI-TOF) m/z: [M+Na $]^{+}$calcd for $\mathrm{C}_{18} \mathrm{H}_{20} \mathrm{~N}_{2} \mathrm{NaO}_{2} 319.1417$; Found 319.1412.

\section{(7) Scale up and Functionalization}

(a) 1 mmol reaction of $N$-(quinolin-8-yl)but-3-enamide. An oven dried Schlenk was charged with Teflon coated magnetic stir bar, $N$-(quinolin-8-yl)but-3-enamide $212 \mathrm{mg}$ (1 mmol), 1,2diphenylethyne 2 a $214 \mathrm{mg}(12 \mathrm{mmol})$ and $\mathrm{Pd}(\mathrm{OAC})_{2}$ catalyst $(10 \mathrm{~mol} \%)$ were added sequentially, followed by HFIP $(10 \mathrm{~mL})$ and $\mathrm{CH}_{3} \mathrm{COOH}$ (3 equiv.). The closed schlenk tube containing reaction mixture were placed in preheated oil bath at $70^{\circ} \mathrm{C}$ for 7 hours. The completion of the reaction was confirmed by checking TLC under UV detector. Then, the organic phase was evaporated under reduced pressure and the product was purified by using silica-gel Column Chromatography (eluent: Hexane/Ethyl acetate =9/1) afforded 3a with 82\% (370 mg) as white solid.

\section{(b) Hydroxylation of alkenyl ester}

Compound $4 \mathbf{h}$ (0.1 mmol), LiOH. $\mathrm{H}_{2} \mathrm{O}\left(0.1 \mathrm{mmol}, 1.0\right.$ equiv) and $\mathrm{MeOH} / \mathrm{H}_{2} \mathrm{O}(300 \mu \mathrm{L} / 100 \mu \mathrm{L})$ were added into a sealed cap reaction tube. The reaction mixture was stirred at room temperature for $10 \mathrm{~min}$, then heated at $100^{\circ} \mathrm{C}$ for 4 hours. After being cooled to room temperature, the mixture was filtered through a pad of celite and concentrated in vacuo. The residue was diluted with $\mathrm{EA}(5 \mathrm{~mL})$ and washed with $\mathrm{NaHCO}_{3}(5 \mathrm{~mL} \times 2)$ and brine $(5 \mathrm{~mL})$, dried over $\mathrm{Na}_{2} \mathrm{SO}_{4}$ and concentrated in vacuo. The resulting residue was purified by silica gel flash chromatography to give compound $\mathbf{8}$ in $92 \%$ yield (37.6 mg).<smiles>O=C(CCC(C(=O)c1ccccc1)c1ccccc1)NO</smiles>

$8,92 \%$

\section{6-oxo-5,6-diphenyl-N-(quinolin-8-yl)hexanamide (8)}

$37.6 \mathrm{mg}, 92 \%$ yield, white solid, m.p $=175-178^{\circ} \mathrm{C}$.

$\boldsymbol{R}_{\boldsymbol{f}}: 0.33$ (hexane/ethyl acetate, $9: 1 \mathrm{v} / \mathrm{v}$ ).

IR (CHCl $\mathbf{3})$ v 3380,3090, 2992, 2870, 2690, 2470, 2442, 1975, 1890, 1712, 1686, 1657, 1564, $1555,1470,1360,1370,1290,1086,985,860,710,540,465 \mathrm{~cm}^{-1}$. 
${ }^{1}$ H NMR (400 MHz, CDCl $) \delta 9.78(\mathrm{~s}, 1 \mathrm{H}), 8.78(\mathrm{dd}, J=4.2,1.5,1 \mathrm{H}), 8.75(\mathrm{dd}, J=7.1,1.6$, $1 \mathrm{H}), 8.14(\mathrm{dd}, J=8.3,1.5,1 \mathrm{H}), 8.00-7.91(\mathrm{~m}, 2 \mathrm{H}), 7.54-7.42(\mathrm{~m}, 4 \mathrm{H}), 7.39-7.26(\mathrm{~m}, 6 \mathrm{H})$, $7.19(\mathrm{t}, J=7.1,1 \mathrm{H}), 4.63(\mathrm{t}, J=7.2,1 \mathrm{H}), 2.66-2.49(\mathrm{~m}, 2 \mathrm{H}), 2.31(\mathrm{tdd}, J=12.2,7.0,5.2$, $1 \mathrm{H}), 2.07-1.93(\mathrm{~m}, 1 \mathrm{H}), 1.91-1.74(\mathrm{~m}, 2 \mathrm{H})$.

${ }^{13}$ C NMR (100 MHz, $\left.\mathbf{C D C l}_{3}\right) \delta$ 199.82, 171.45, 148.23, 139.45, 138.39, 136.83, 136.47, $134.55,133.00,129.10,128.81,128.62$, 128.35, 128.02, 127.50, 127.23, 121.70, 121.53, $116.52,53.72,38.17,33.59,23.89$.

HRMS (ESI-TOF) m/z: [M+H] $]^{+}$calcd for $\mathrm{C}_{27} \mathrm{H}_{25} \mathrm{~N}_{2} \mathrm{O}_{2} 409.1911$; Found 409.1914.

\section{(c) Removal of Direction group}

In an oven dried reaction tube $0.1 \mathrm{mmol}$ of $\mathbf{4 f}$ was taken. Then 3 equiv $p$-toluenesulphonic acid and $1 \mathrm{~mL}$ methanol was added to it. The mixture was stirred in a preheated oil bath at $90{ }^{\circ} \mathrm{C}$ for 24 hours. After completion reaction was cooled to room temperature, filtered through celite and evaporated under vacuum. Pure product was isolated by column chromatography through a silica gel column (mesh 100- 200). Eluent: ethyl acetate / petroleum ether (5:95 v/v); colourless liquid; isolated yield: $96 \%(47.1 \mathrm{mg})$.<smiles>COC(=O)CC(CC(=C(c1ccccc1)c1ccccc1)c1ccccc1)C(=O)OC(=O)C(c1ccccc1)c1ccccc1</smiles>

\section{methyl (Z)-6-(2,2-diphenylacetoxy)-5,6-diphenylhex-5-enoate $(9)$}

$47.1 \mathrm{mg}, 96 \%$ yield, white solid, m.p $=165-170{ }^{\circ} \mathrm{C}$.

$\boldsymbol{R}_{\boldsymbol{f}}: 0.33$ (hexane/ethyl acetate, $9: 1 \mathrm{v} / \mathrm{v}$ ).

IR (CHCl $)$ : v 3370,3080, 2982, 2897, 2687, 2465, 2439, 1972, 1885, 1805, 1745, 1736, 1653, $1554,1525,1462,1350,1310,1285,1078,990,879,725,545,485 \mathrm{~cm}^{-1}$.

${ }^{1}$ H NMR (400 MHz, $\left.\mathbf{C D C l}_{3}\right) \delta 7.31(\mathrm{~s}, J=6.0,5 \mathrm{H}), 7.28(\mathrm{dd}, J=6.9,3.7,3 \mathrm{H}), 7.24-7.14$ (m, 8H), $6.92(\mathrm{dd}, J=7.3,2.1,4 \mathrm{H}), 4.80$ (s, $J=97.9,1 \mathrm{H}), 3.55$ (s, 3H), 2.47 (dd, $J=8.8,6.9$, $2 \mathrm{H}), 2.21(\mathrm{t}, J=7.5,2 \mathrm{H}), 1.72-1.61(\mathrm{~m}, 2 \mathrm{H})$.

${ }^{13}$ C NMR (100 MHz, $\left.\mathbf{C D C l}_{3}\right) \delta 173.69,170.80,143.32,138.14,138.01,135.02,130.76$, 129.12 , 128.76, 128.56, 128.53, 128.43, 128.24, 128.21, 127.36, 127.16, 56.85, 51.58, 33.57, $32.52,23.55$.

HRMS (ESI-TOF) m/z: [M+Na] $]^{+}$calcd for $\mathrm{C}_{33} \mathrm{H}_{30} \mathrm{NaO}_{4}$ 513.2036; Found 513.2031. 


\section{8) References}

1) (a) Gurak, J. A., Jr.; Yang, K. S.; Liu, Z.; Engle, K. M. J. Am. Chem. Soc. 2016, 138, 5805.

(b) Yang, K. S.; Gurak, J. A., Jr.; Liu, Z.; Engle, K. M. J. Am. Chem. Soc. 2016, 138, 14705.

2) Singh, A.; Shukla, R. K.; Volla, C. M. R. Chem. Commun., 2019, 55, 13442.

3) (a) Zheng, Q.; Hua, R.; Wan, Y. Appl. Organomet. Chem. 2010, 24, 314. (b) Vilhelmsen, M. H.; Jensen, J.; Tortzen, C. G.; Nielsen, M. B. Eur. J. Org. Chem. 2013, 701.

4) Hofmeister, B. H.; Annen, K.; Laurent, H.; Wiechert, R. Angew. Chem. Int. Ed. Engl. 1984, 23,727 . 
9) ${ }^{1} \mathrm{H}$ and ${ }^{13} \mathrm{C}$ NMR Spectra of the Compounds

CMRV-RS-1046-A-1H
CMRV-RS-1046-A-1H<smiles>C#CCC(CC(=O)NO)CC(=O)OC(C)=O</smiles>

1H NMR (400 MHz, CDCI3)
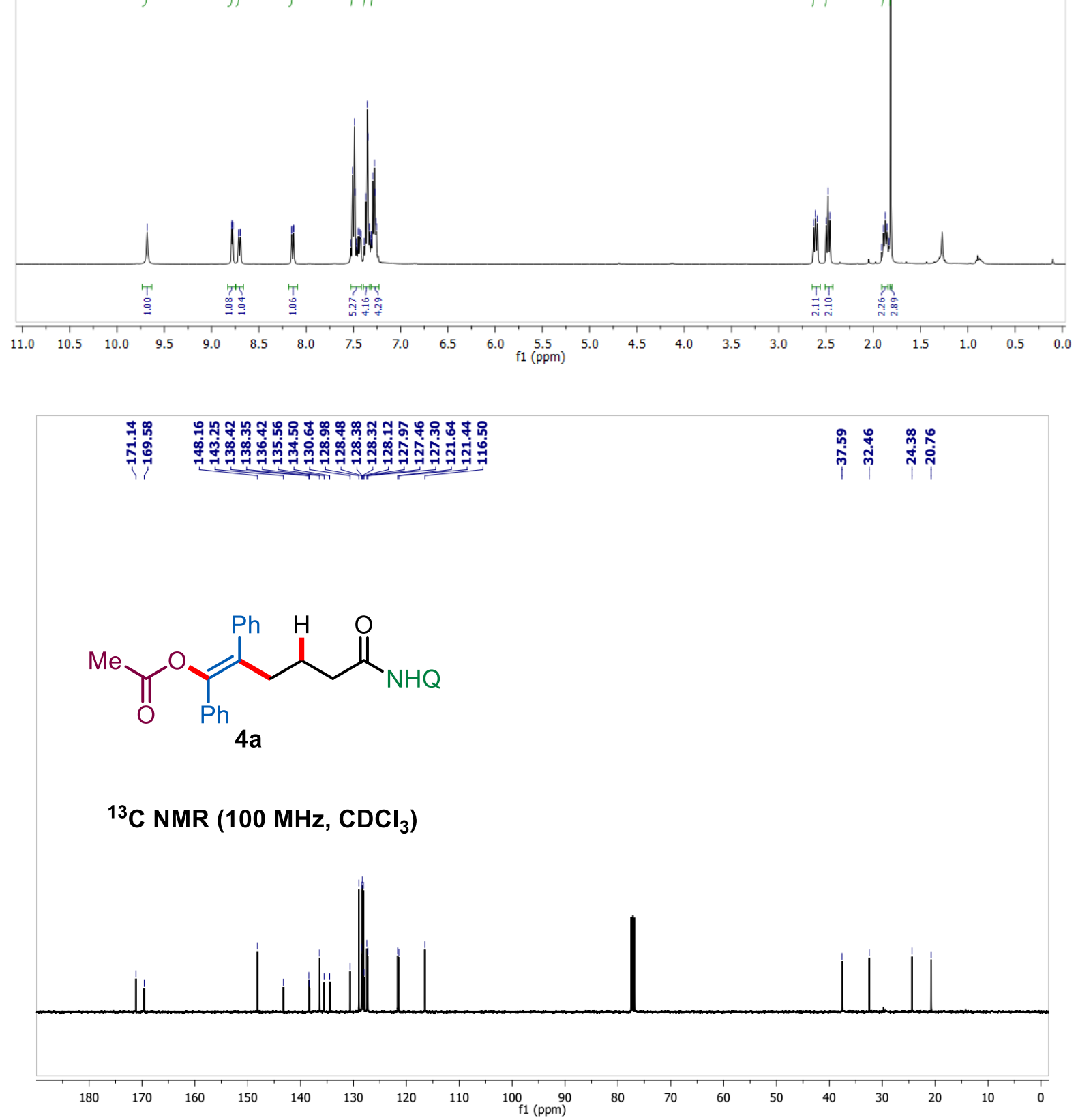
<smiles>C#CCC(CC(=O)NO)CC(Pc1ccccc1)=C(P)OC(=O)CC</smiles>

4b

${ }^{1} \mathrm{H}$ NMR $\left(400 \mathrm{MHz}, \mathrm{CDCl}_{3}\right)$
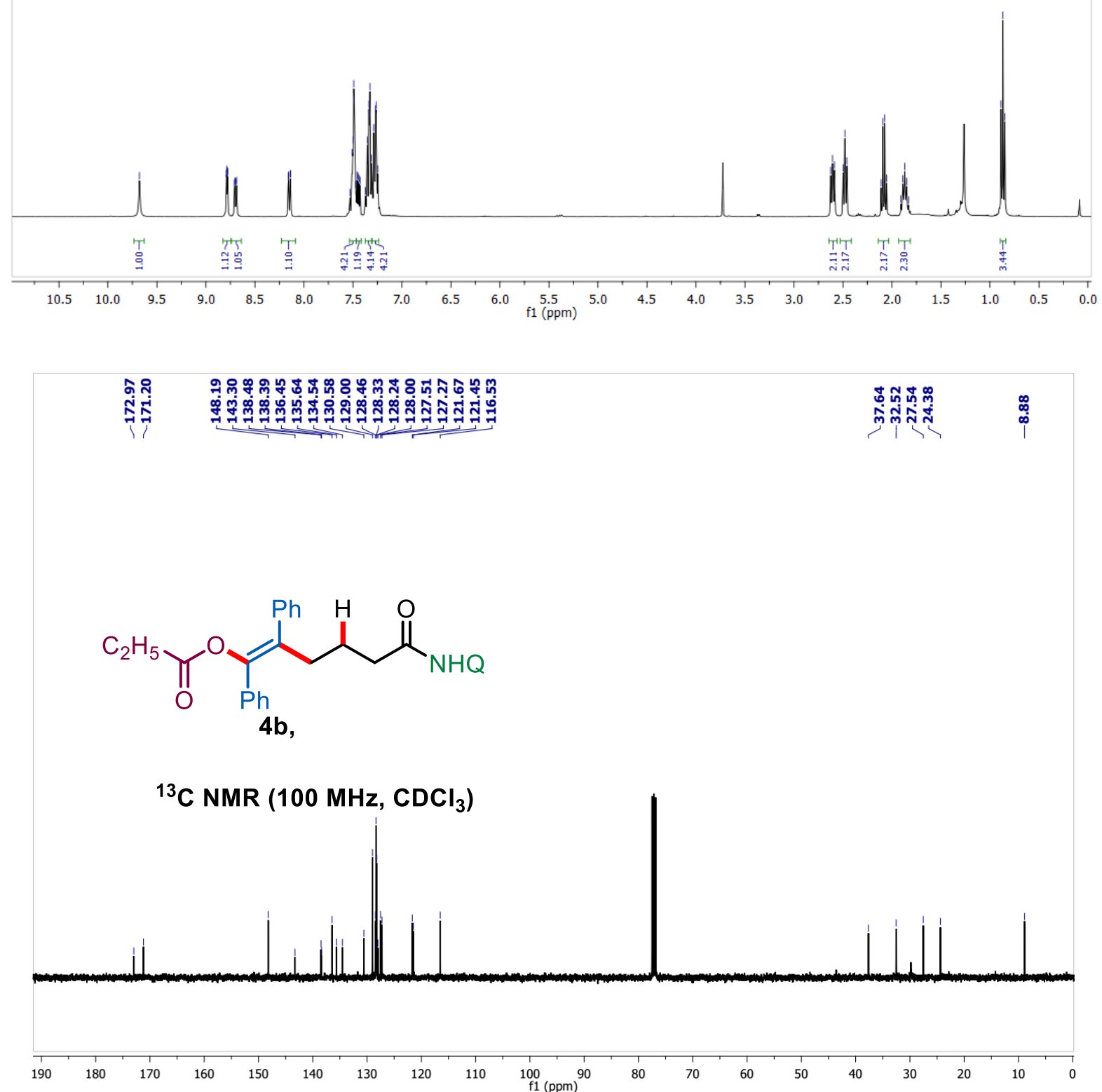


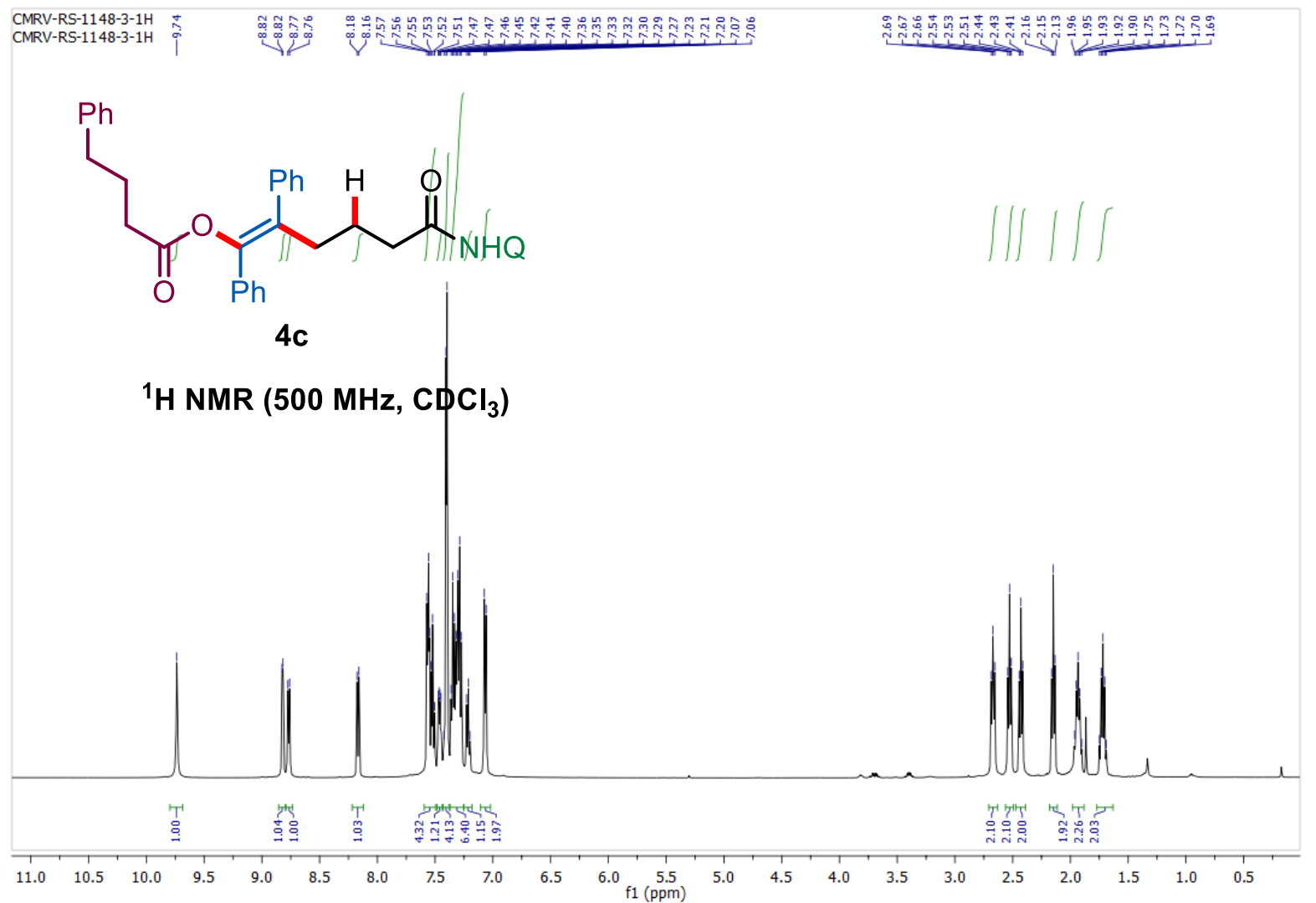

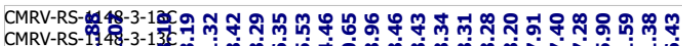

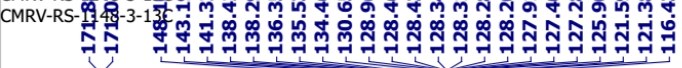

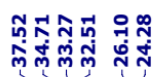<smiles>O=C(CCC(Pc1ccccc1)=C(P)OC(=O)CCCc1ccccc1)NO</smiles>

4c

${ }^{13} \mathrm{C}$ NMR (125 MHz, $\left.\mathrm{CDCl}_{3}\right)$

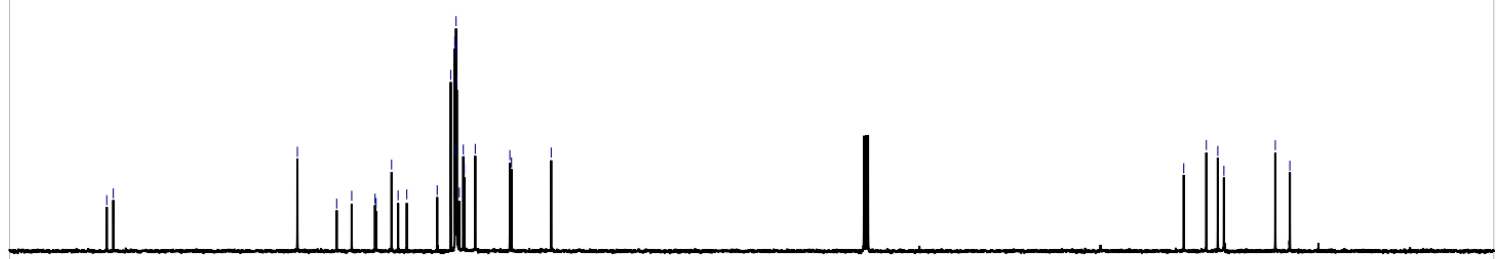

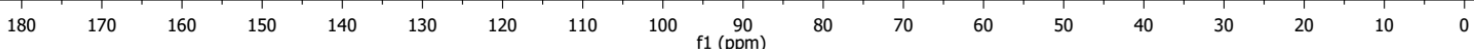




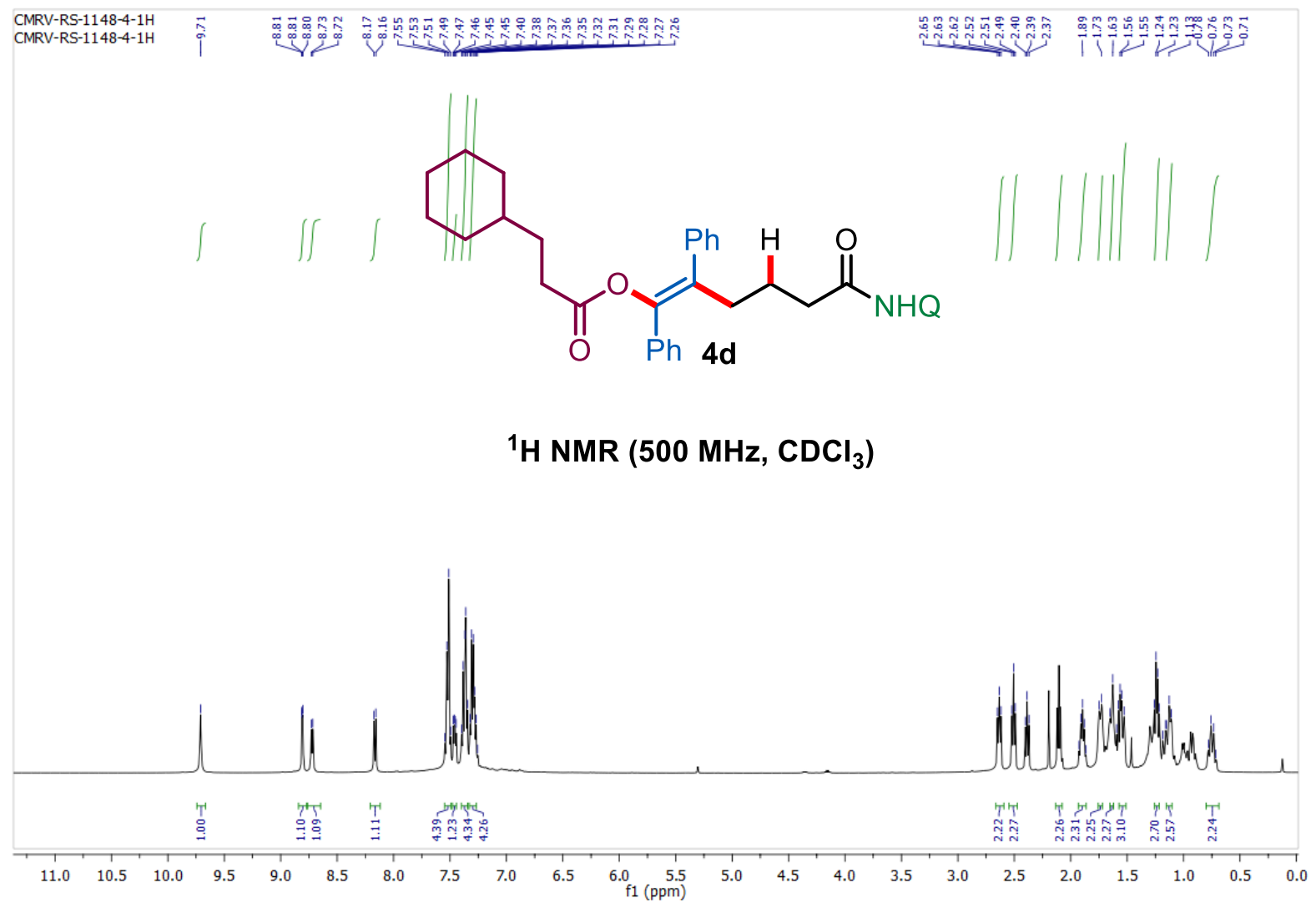

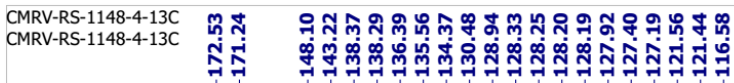

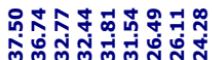

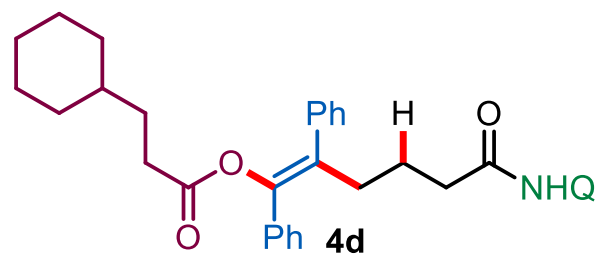

${ }^{13} \mathrm{C}$ NMR (125 MHz, $\left.\mathrm{CDCl}_{3}\right)$
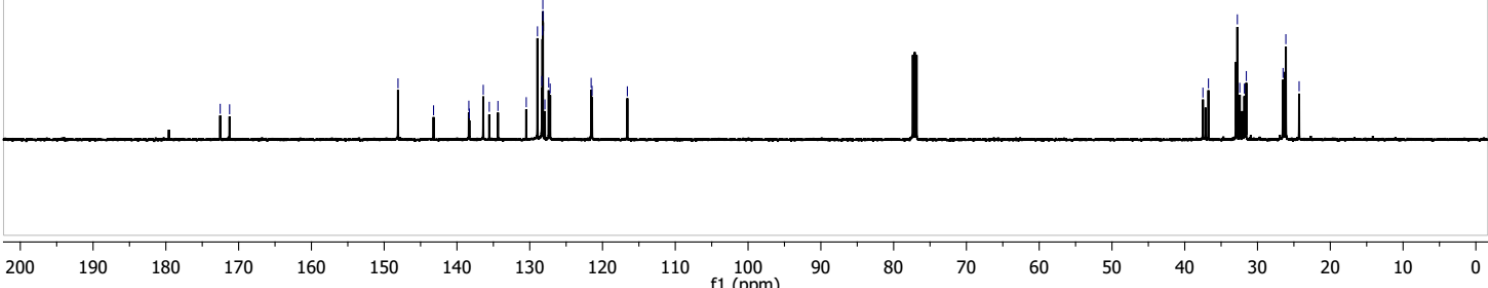

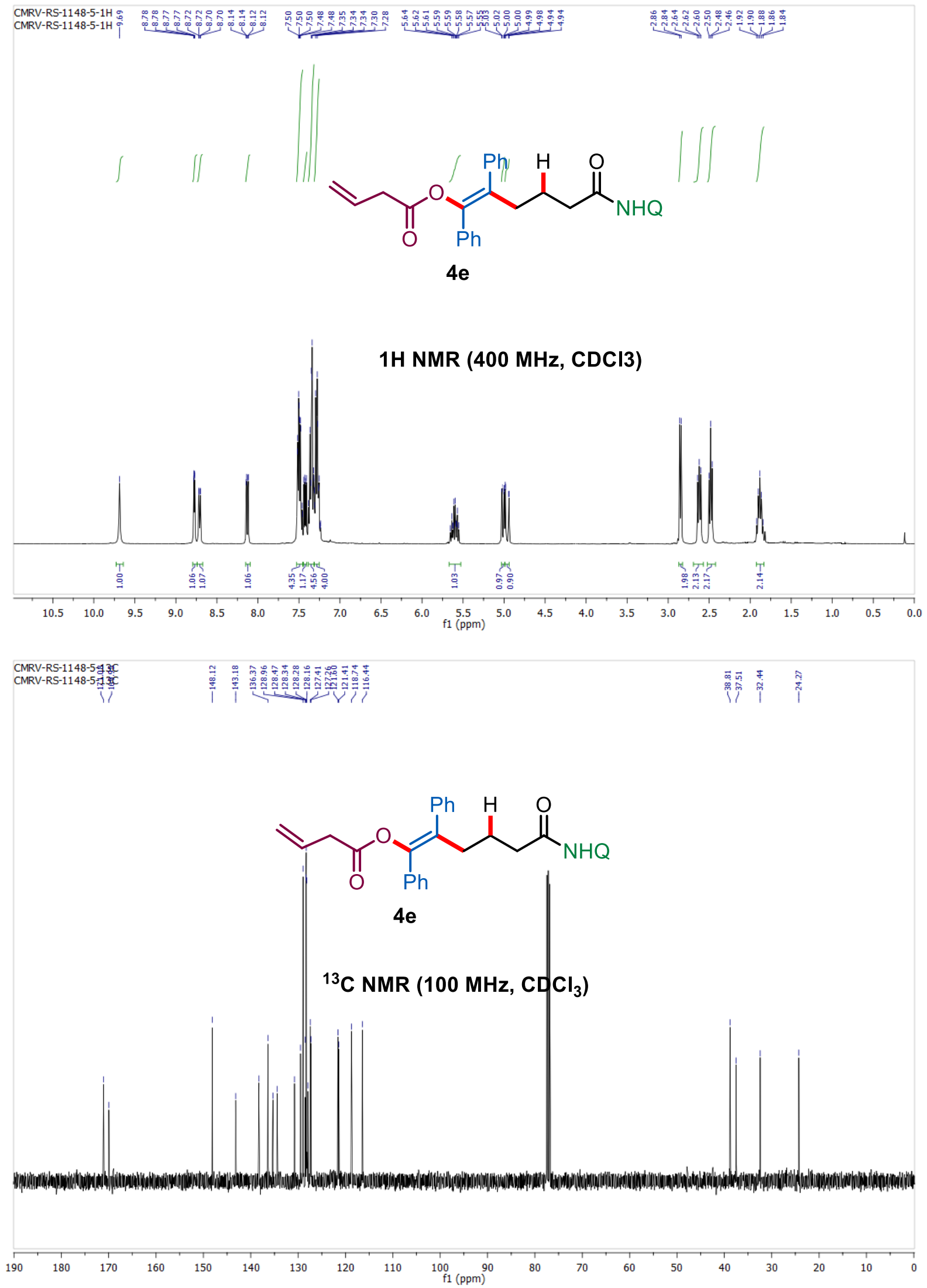


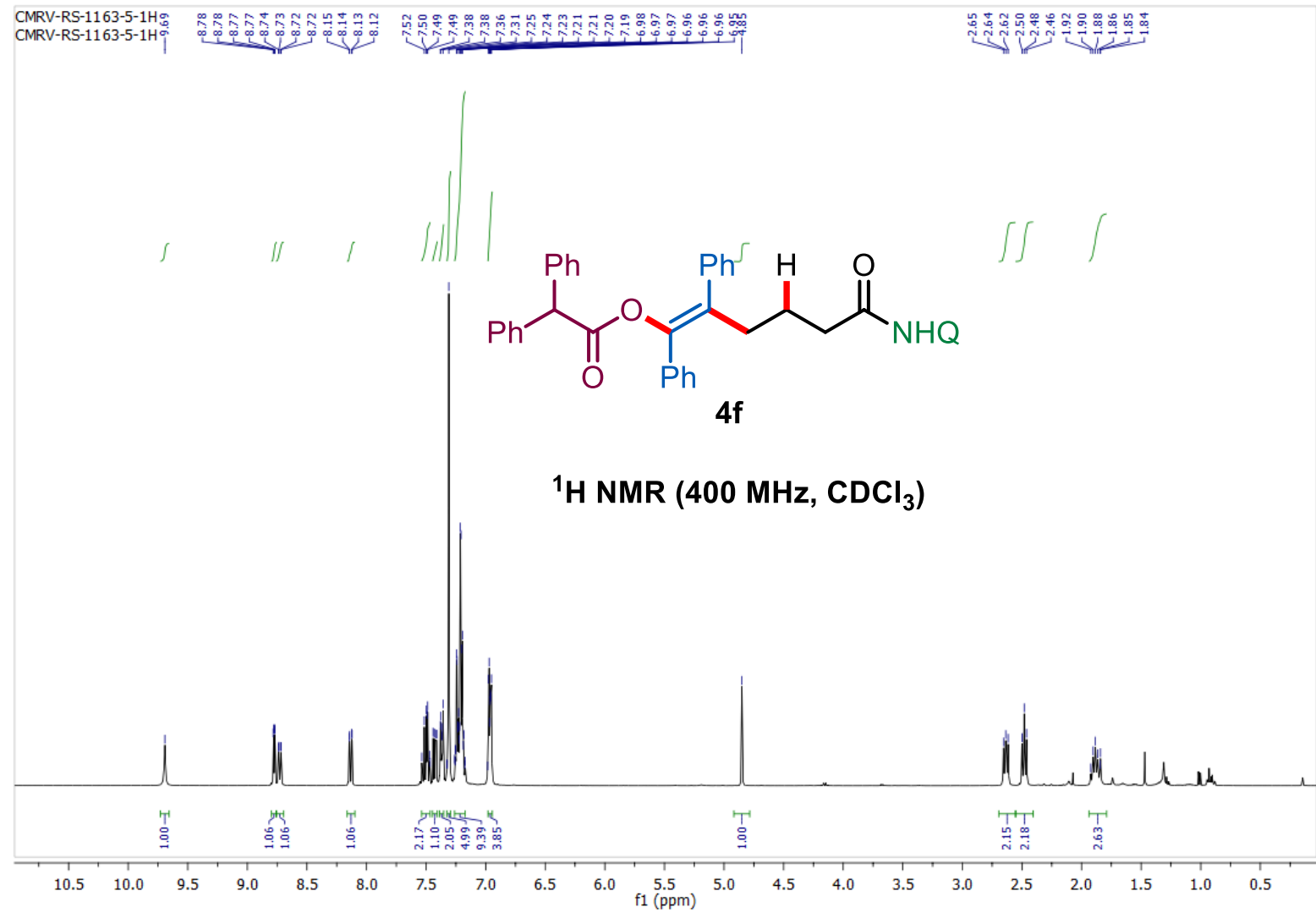

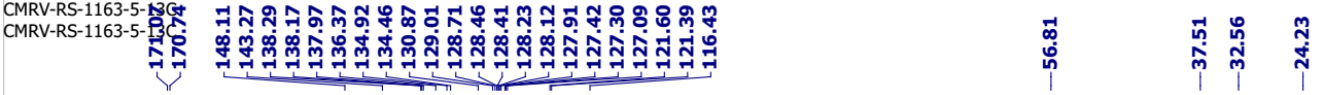<smiles>O=C(C/C=C(\OC(=O)C(c1ccccc1)c1ccccc1)Pc1ccccc1)NO</smiles>

$4 f$

${ }^{13} \mathrm{C}$ NMR (100 $\left.\mathrm{MHz}, \mathrm{CDCl}_{3}\right)$

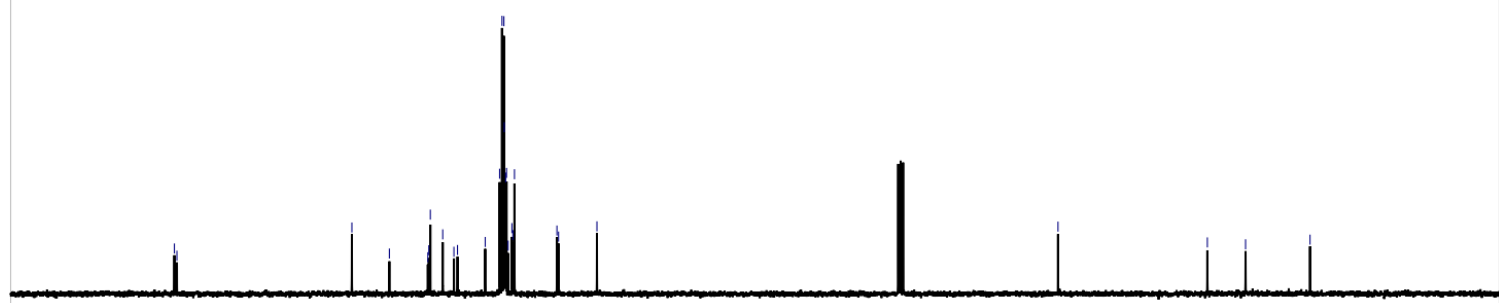

$\begin{array}{lllllllllllllllllllllll}190 & 180 & 170 & 160 & 150 & 140 & 130 & 120 & 110 & 100 & 100 & 80 & 70 & 60 & 50 & 40 & 30 & 20 & 10 & 0\end{array}$ 


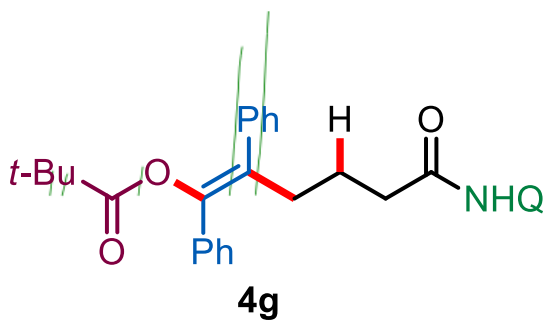

${ }^{1} \mathrm{H}$ NMR (400 MHz, $\left.\mathrm{CDCl}_{3}\right)$

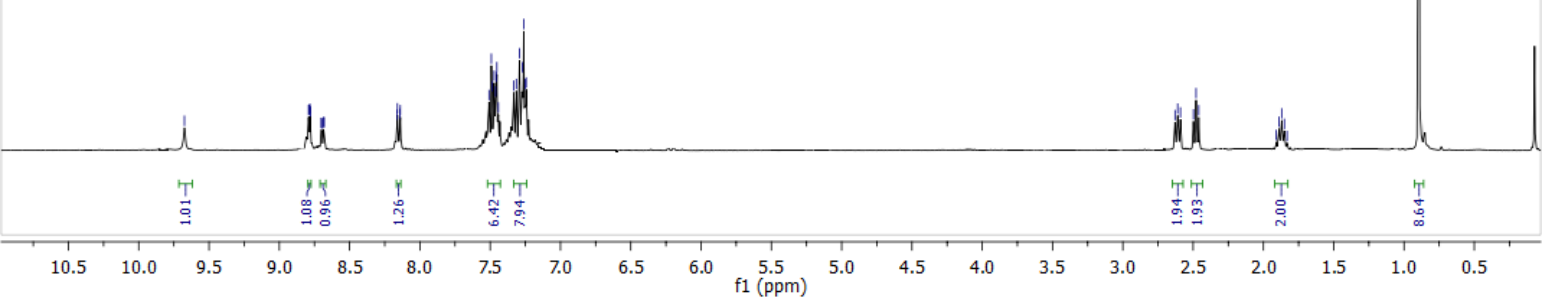

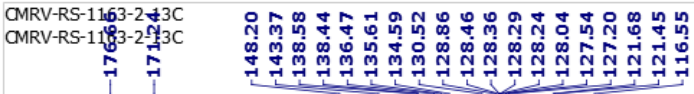

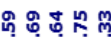

ตัต<smiles>CC(C)(C)C(=O)O/C(Pc1ccccc1)=C(\CCC(=O)NO)c1ccccc1</smiles>

$4 g$

${ }^{13} \mathrm{C}$ NMR (125 MHz, $\left.\mathrm{CDCl}_{3}\right)$

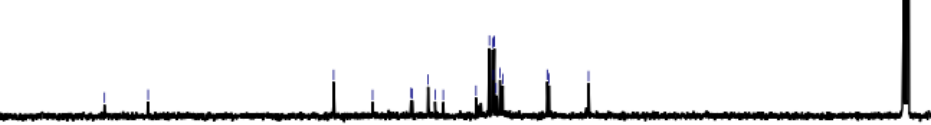

18

$170 \quad 160$
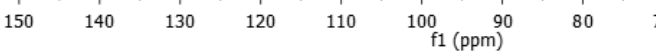

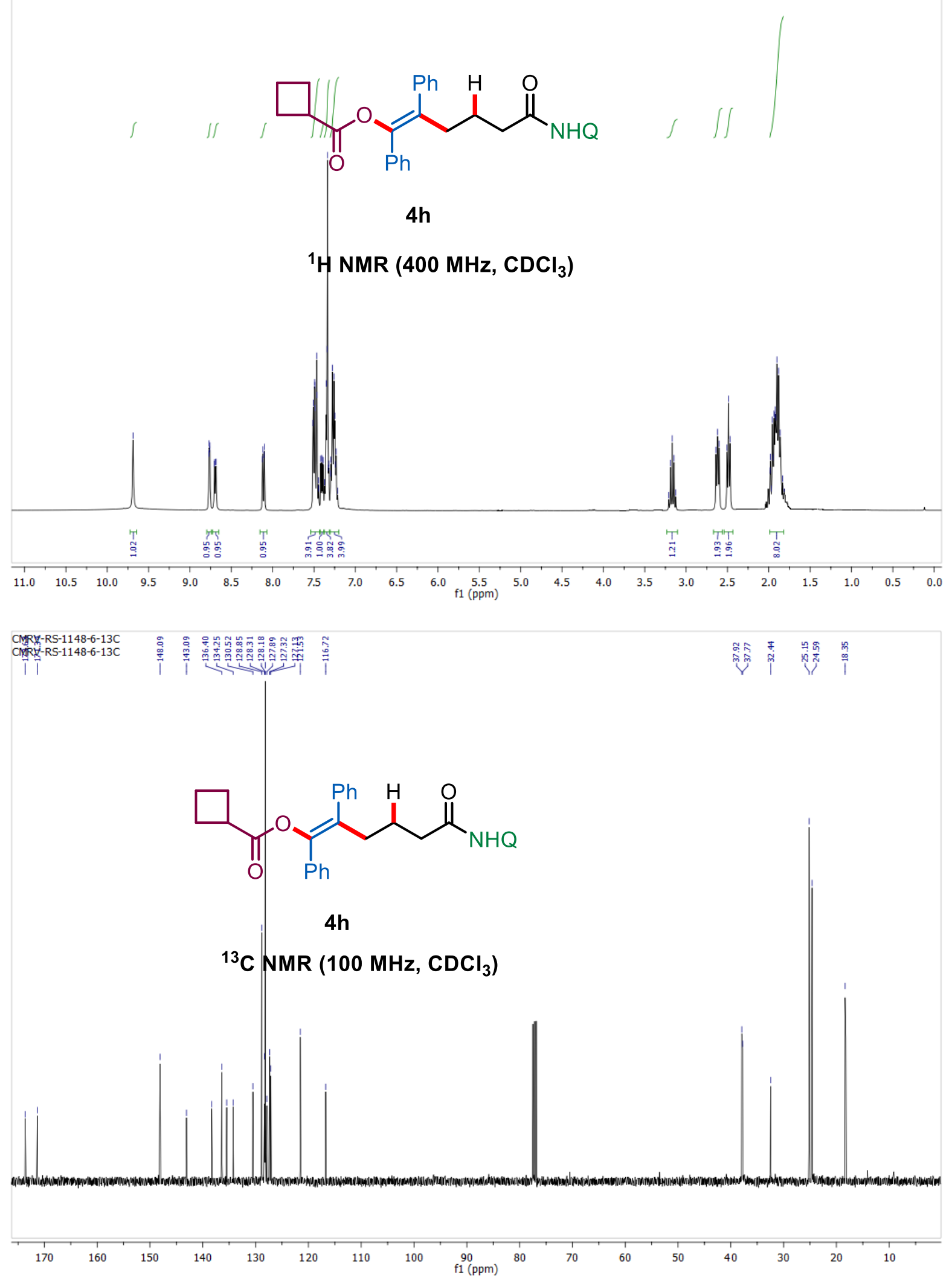

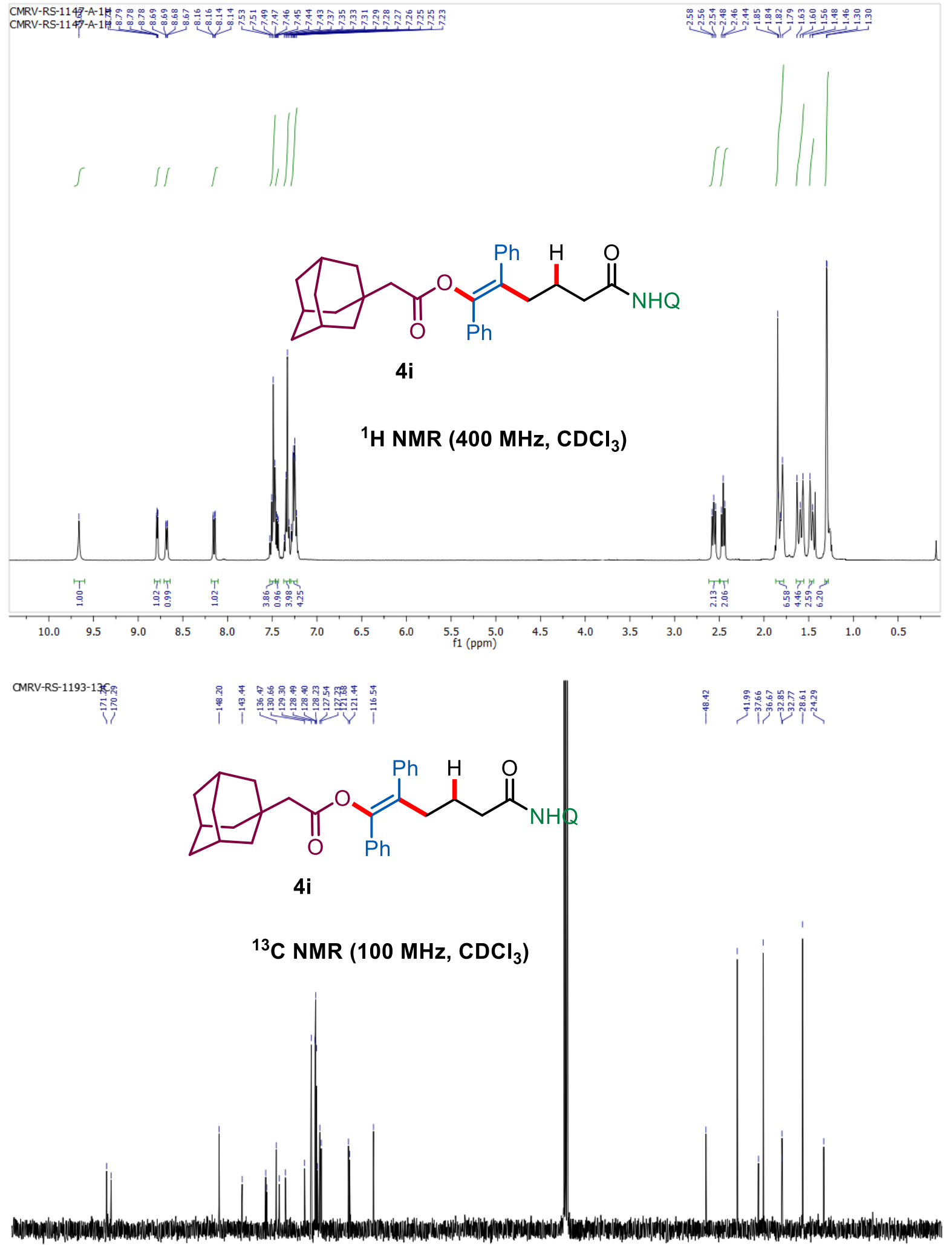

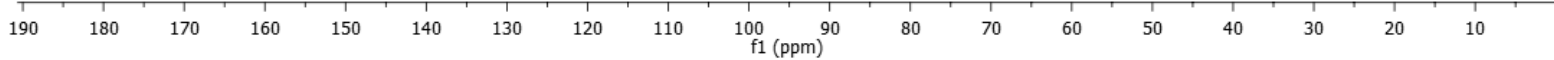



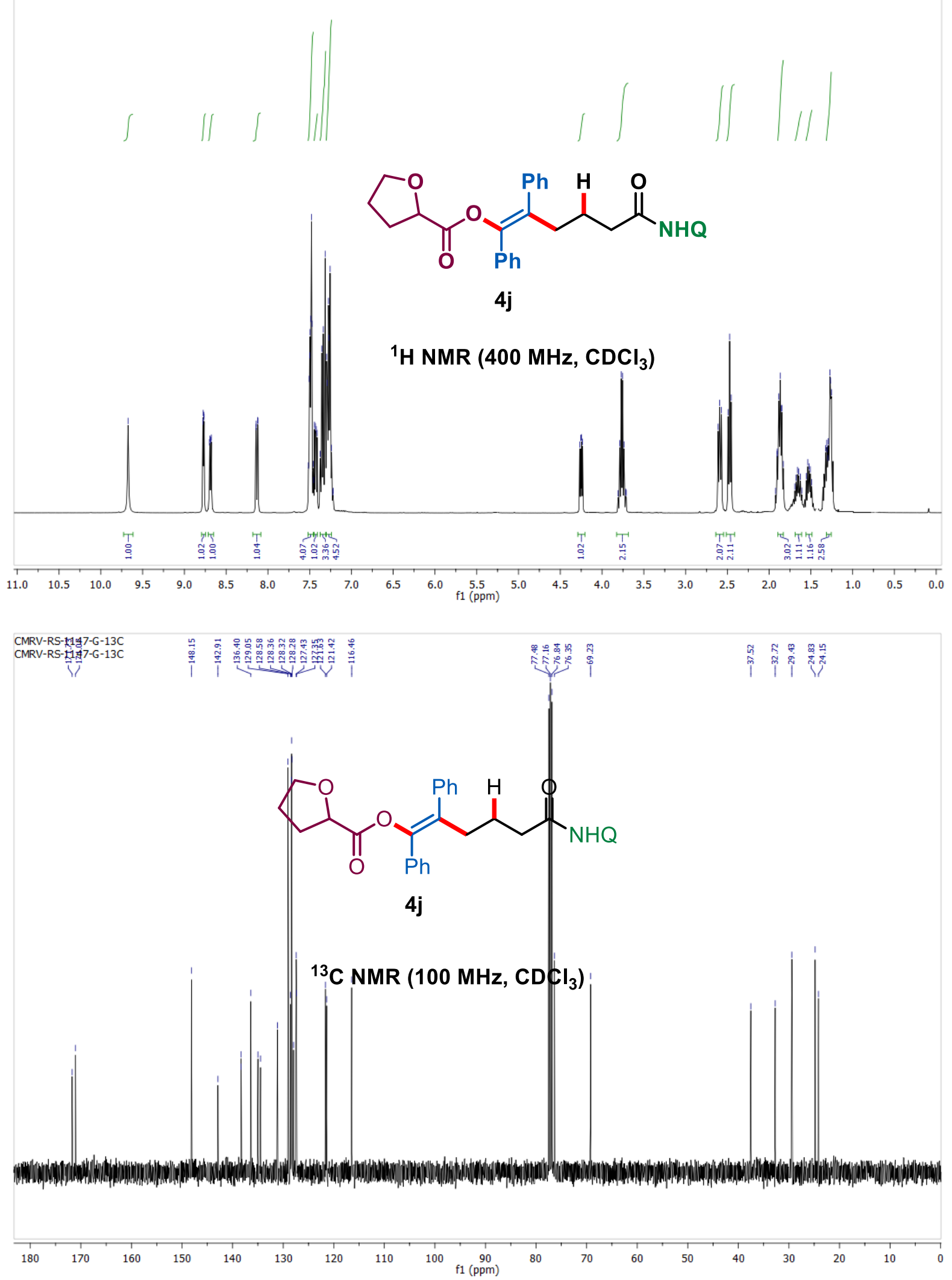

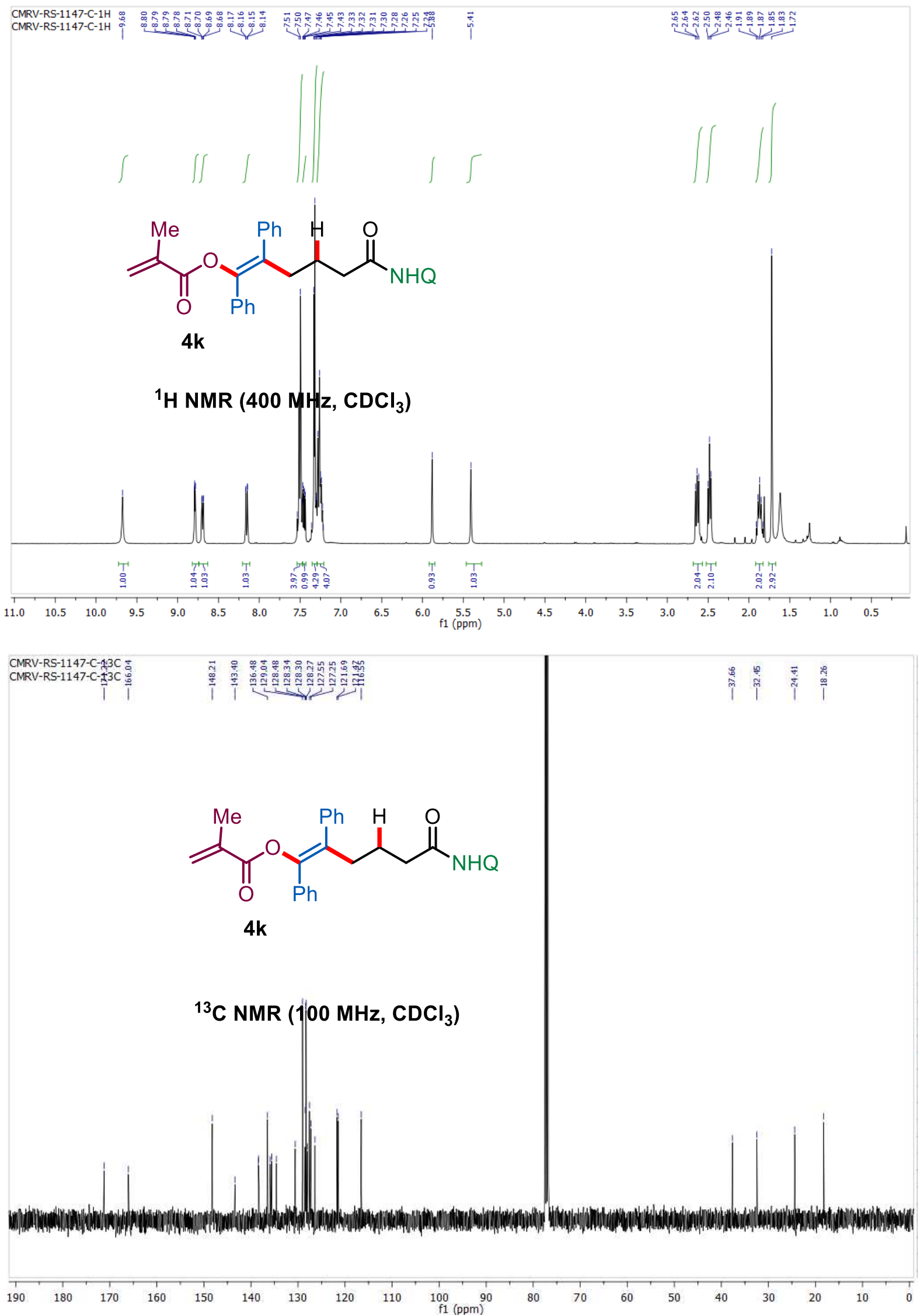


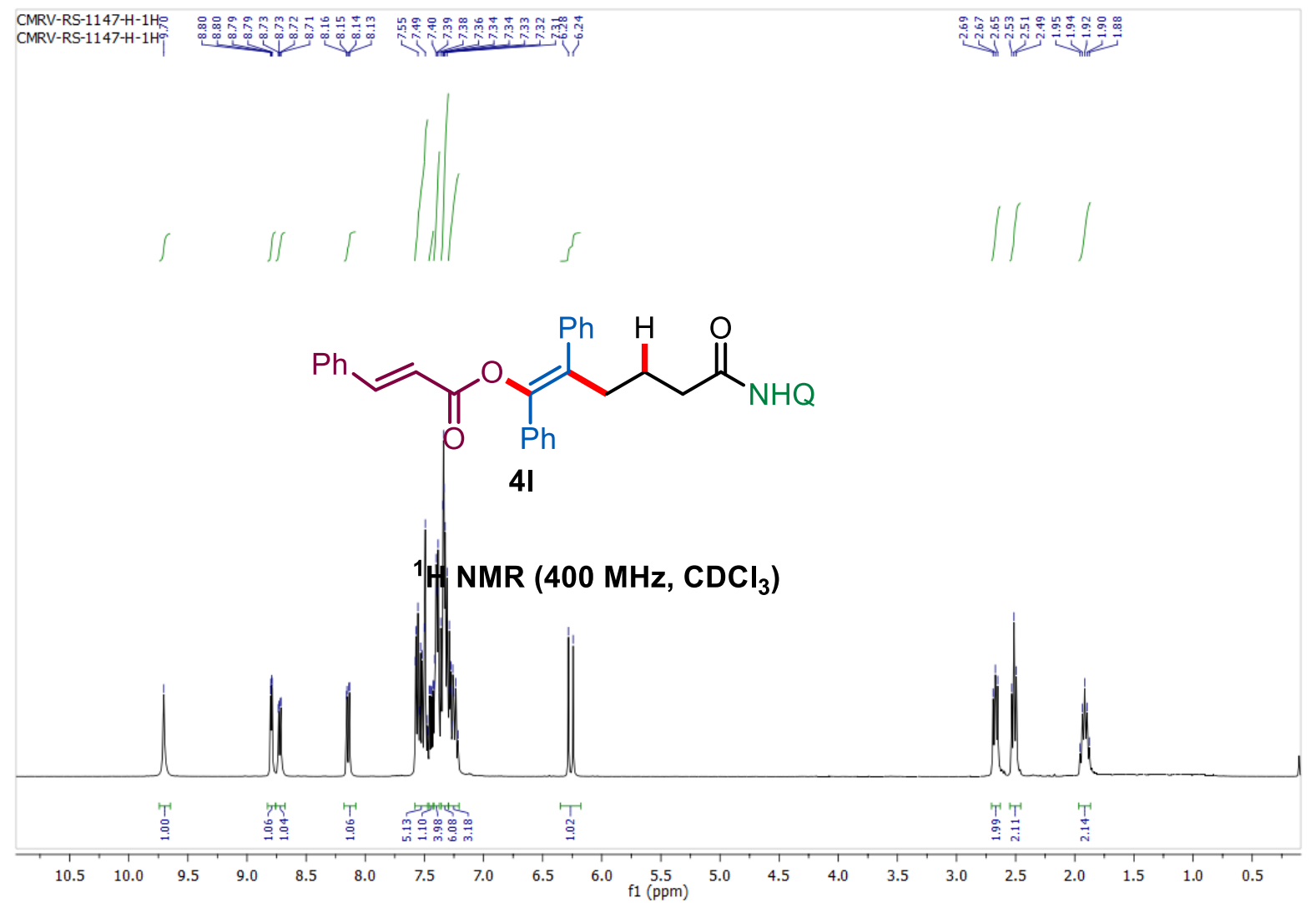

CMRV-RS-1182-AG<smiles>O=C(CCC(P)=C(OC(=O)C=Cc1ccccc1)Pc1ccccc1)NO</smiles>

4I

${ }^{13} \mathrm{C}$ NMR $\left(100 \mathrm{MHz}, \mathrm{CDCl}_{3}\right)$

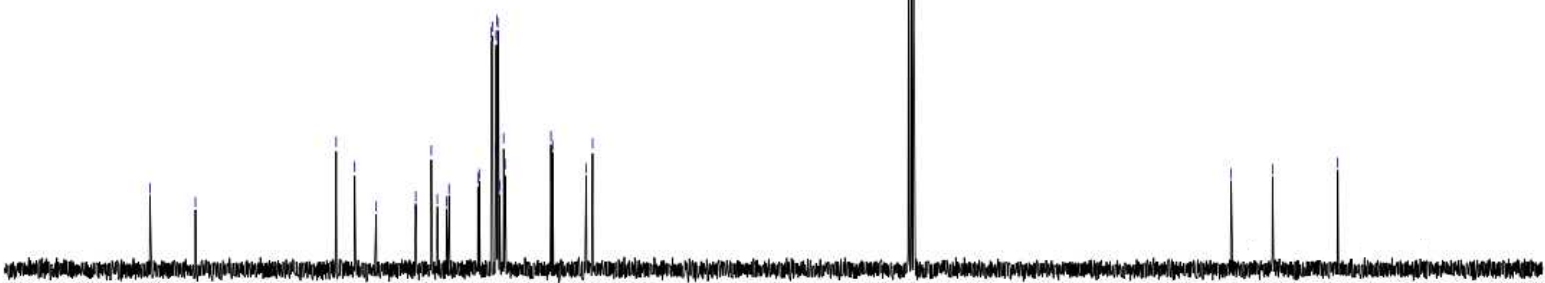

$\begin{array}{lllllllll}180 & 170 & 160 & 150 & 140 & 130 & 120 & 110 & 100 \quad 90 \\ \mathrm{f} 1(\mathrm{ppm})\end{array}$ 

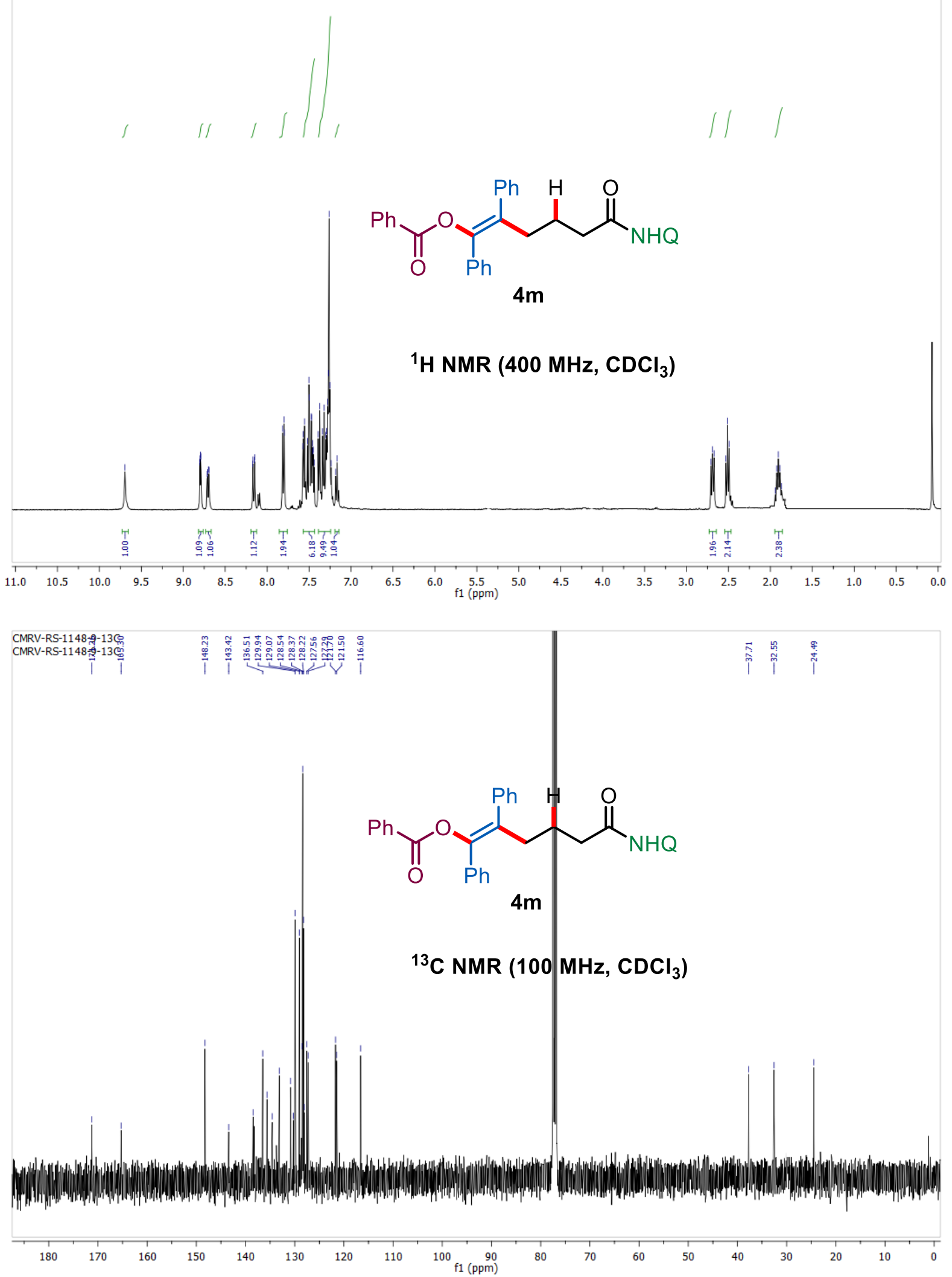


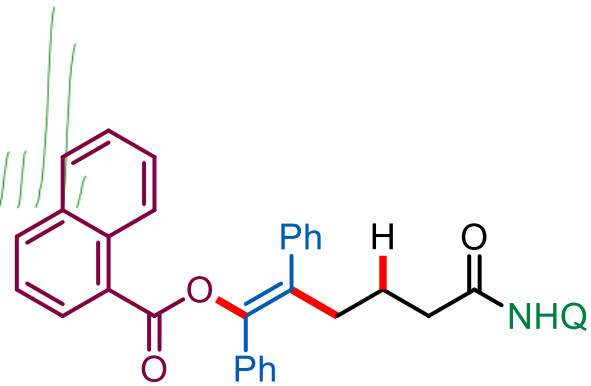

$4 n$

${ }^{1} \mathrm{H}$ NMR (400 MHz, $\left.\mathrm{CDCl}_{3}\right)$

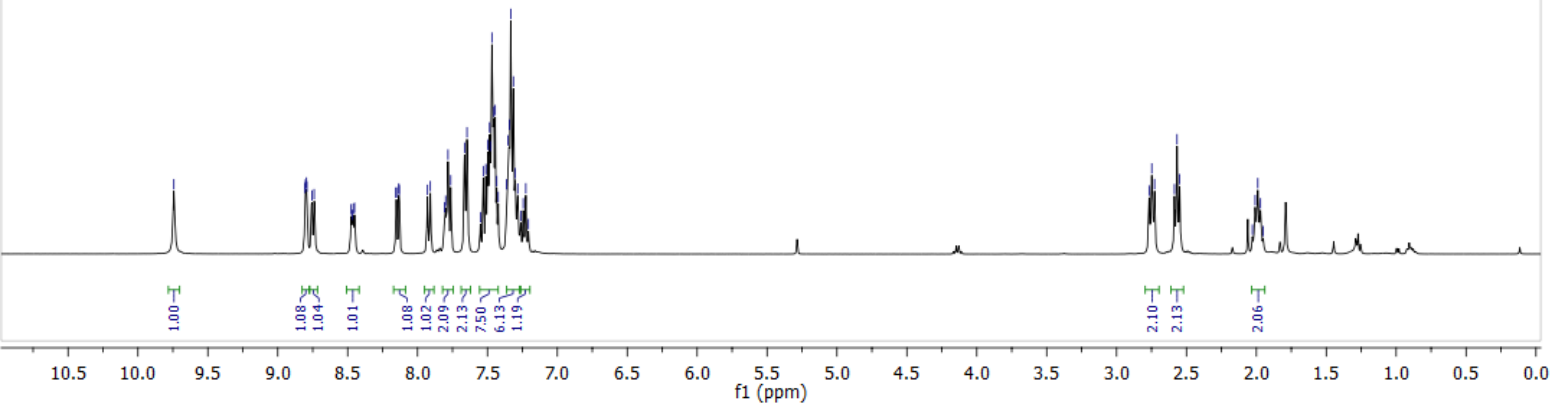

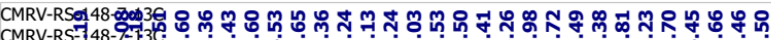

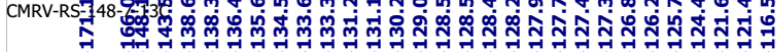

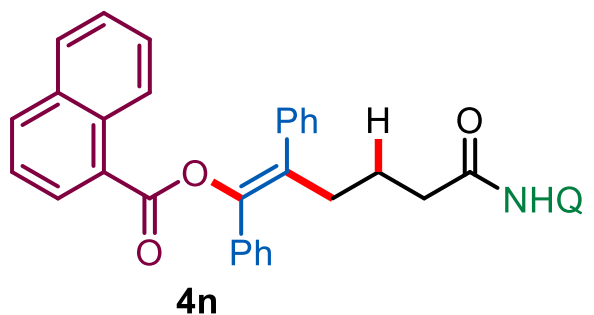

${ }^{13} \mathrm{C}$ NMR $\left(100 \mathrm{MHz}, \mathrm{CDCl}_{3}\right)$

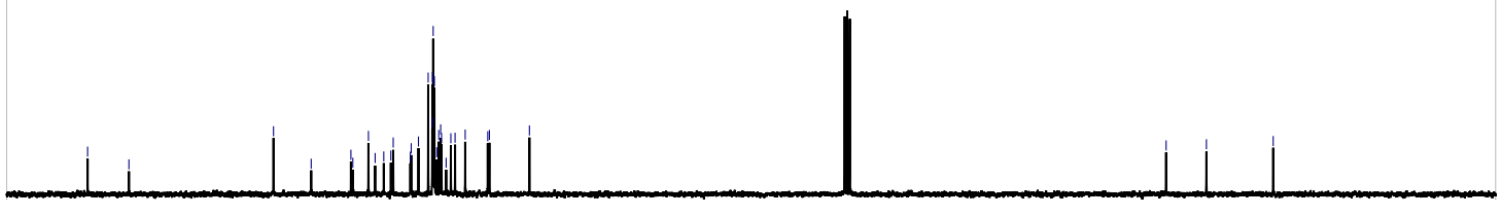



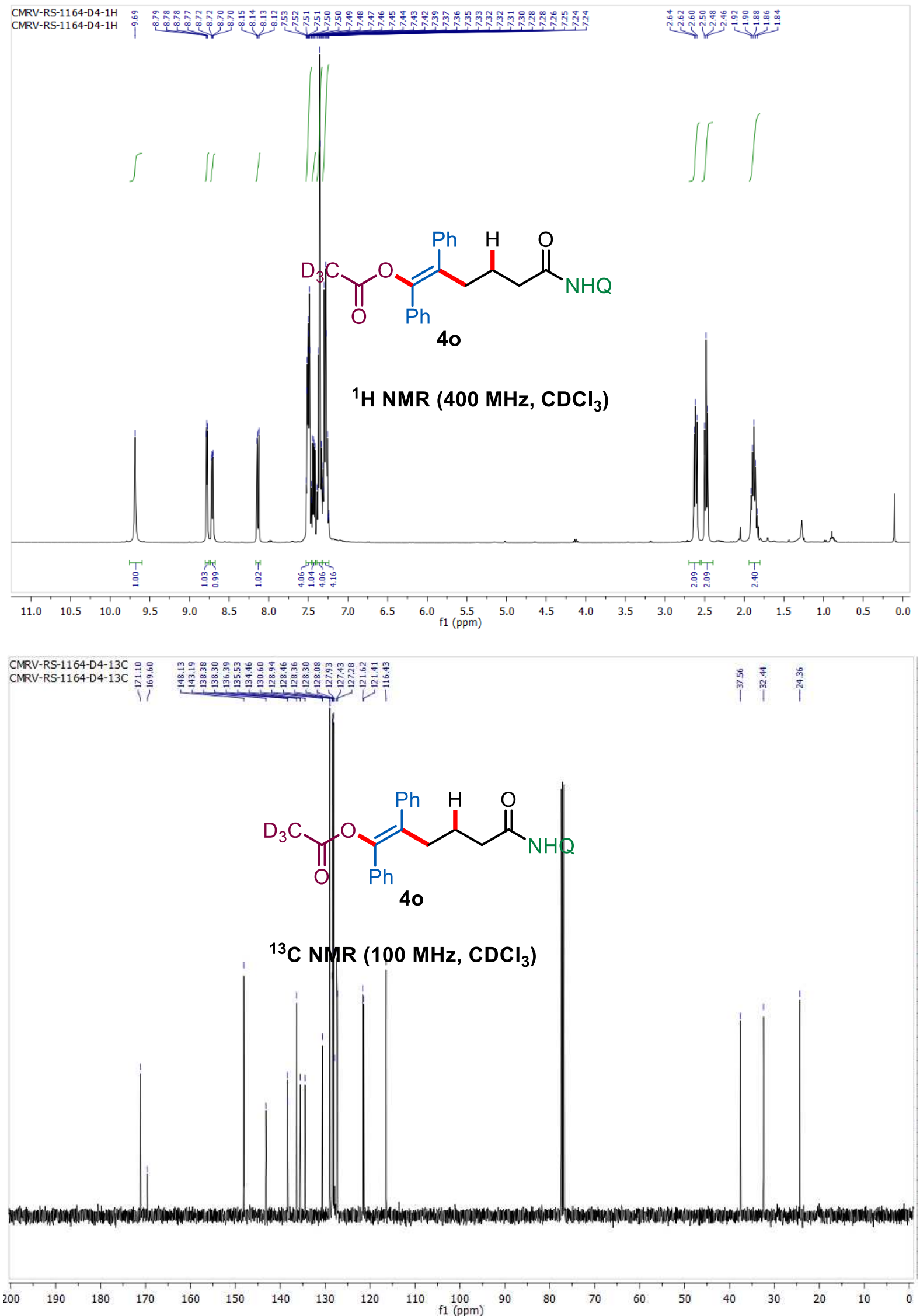

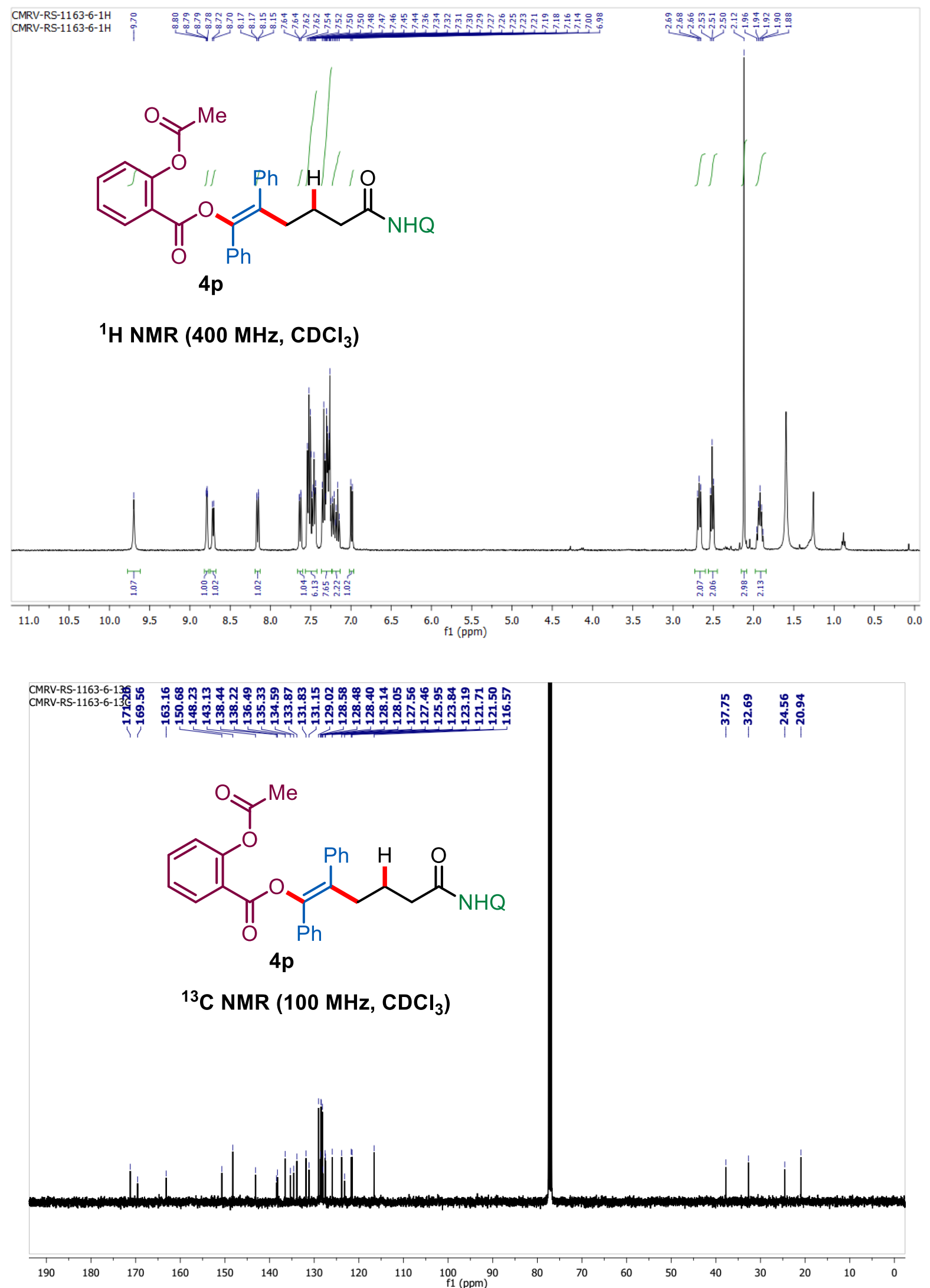

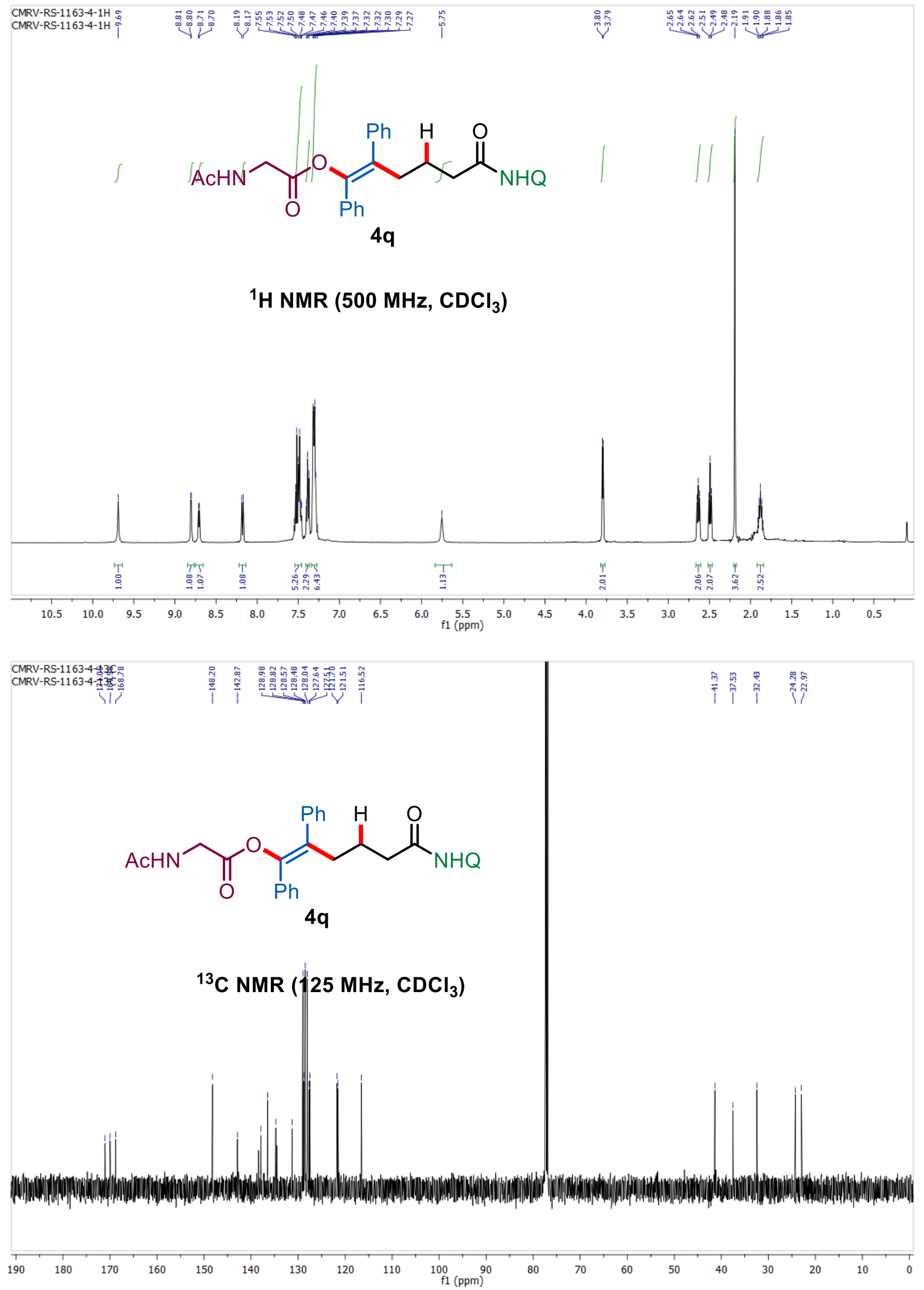
<smiles>CCC(C)[C@H](NP)C(=O)O/C(=C(\CC(CC(=O)NO)c1ccccc1)c1ccccc1)c1ccccc1</smiles>

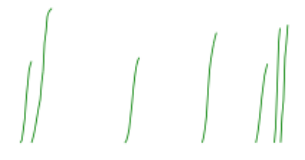

${ }^{1} \mathrm{H}$ NMR (400 MHz, $\left.\mathrm{CDCl}_{3}\right)$
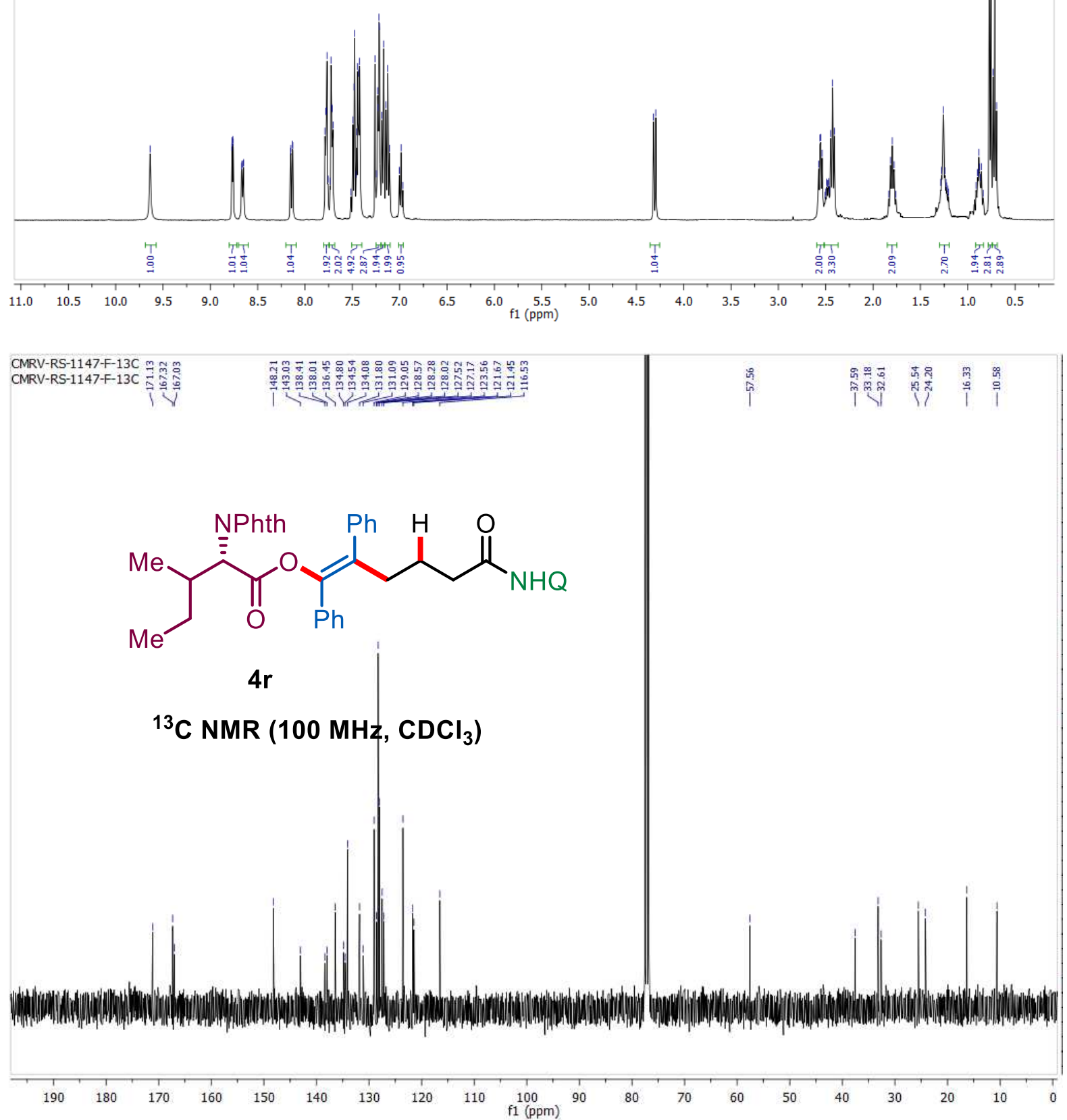

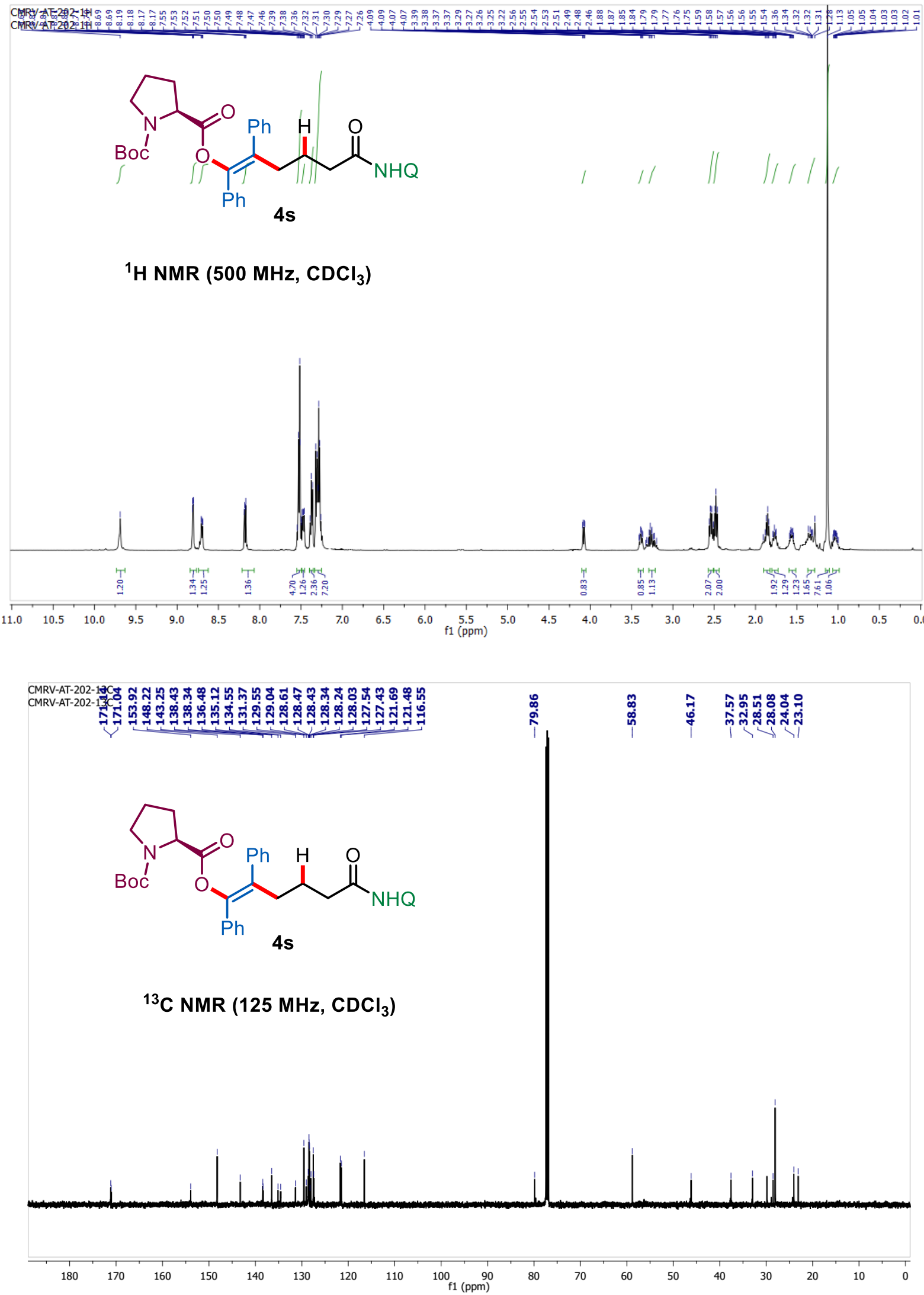


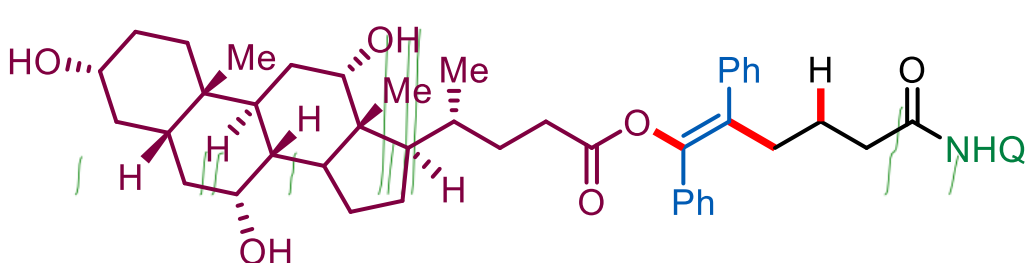

4t

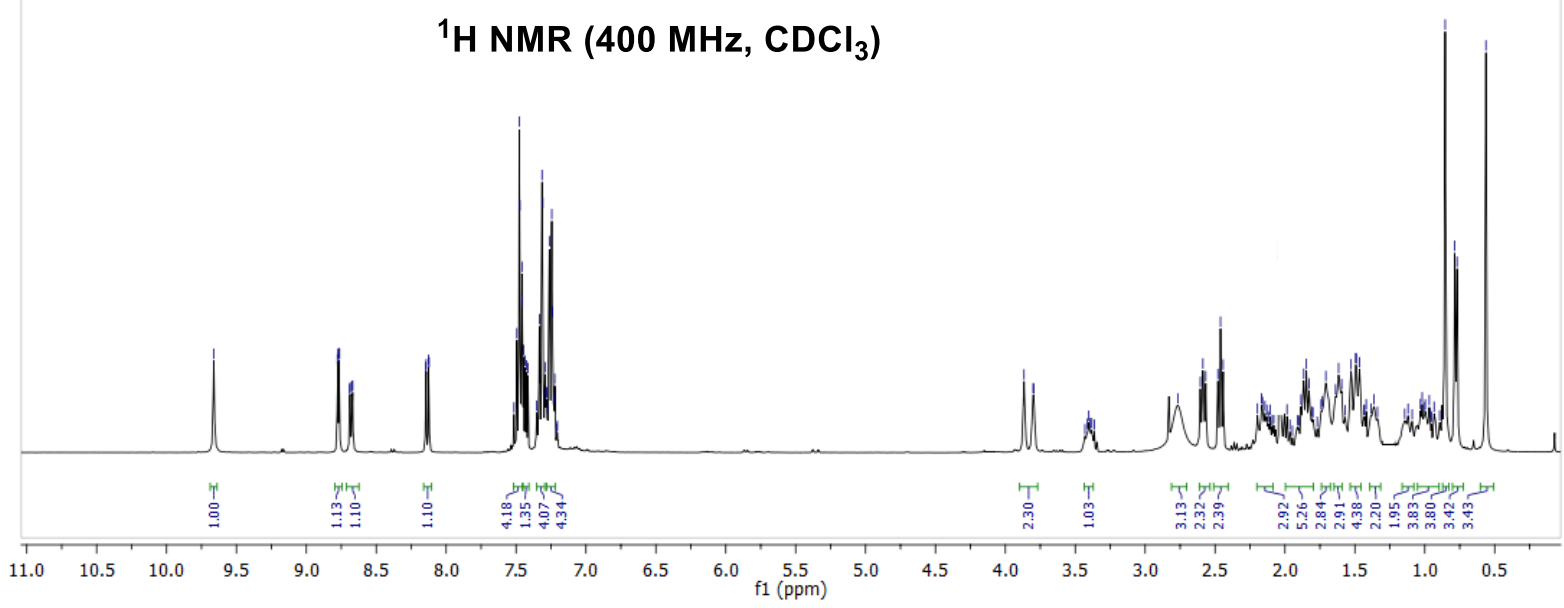

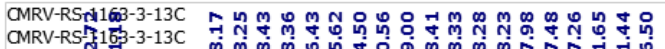

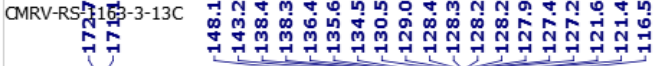

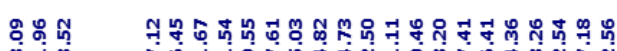

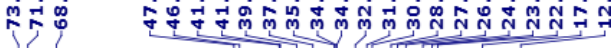

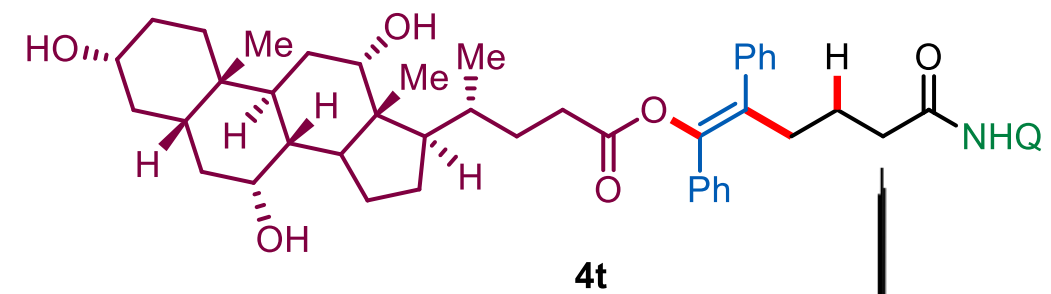

${ }^{13} \mathrm{C}$ NMR (100 MHz, $\left.\mathrm{CDCl}_{3}\right)$

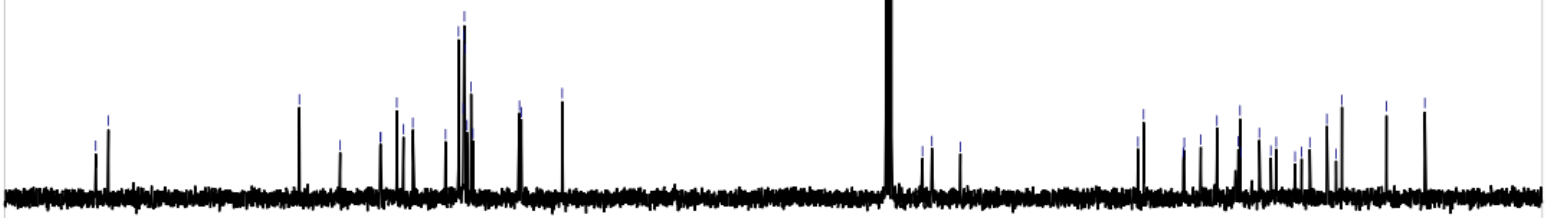

180

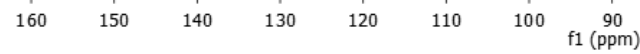

$80 \quad 70$

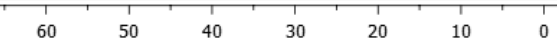



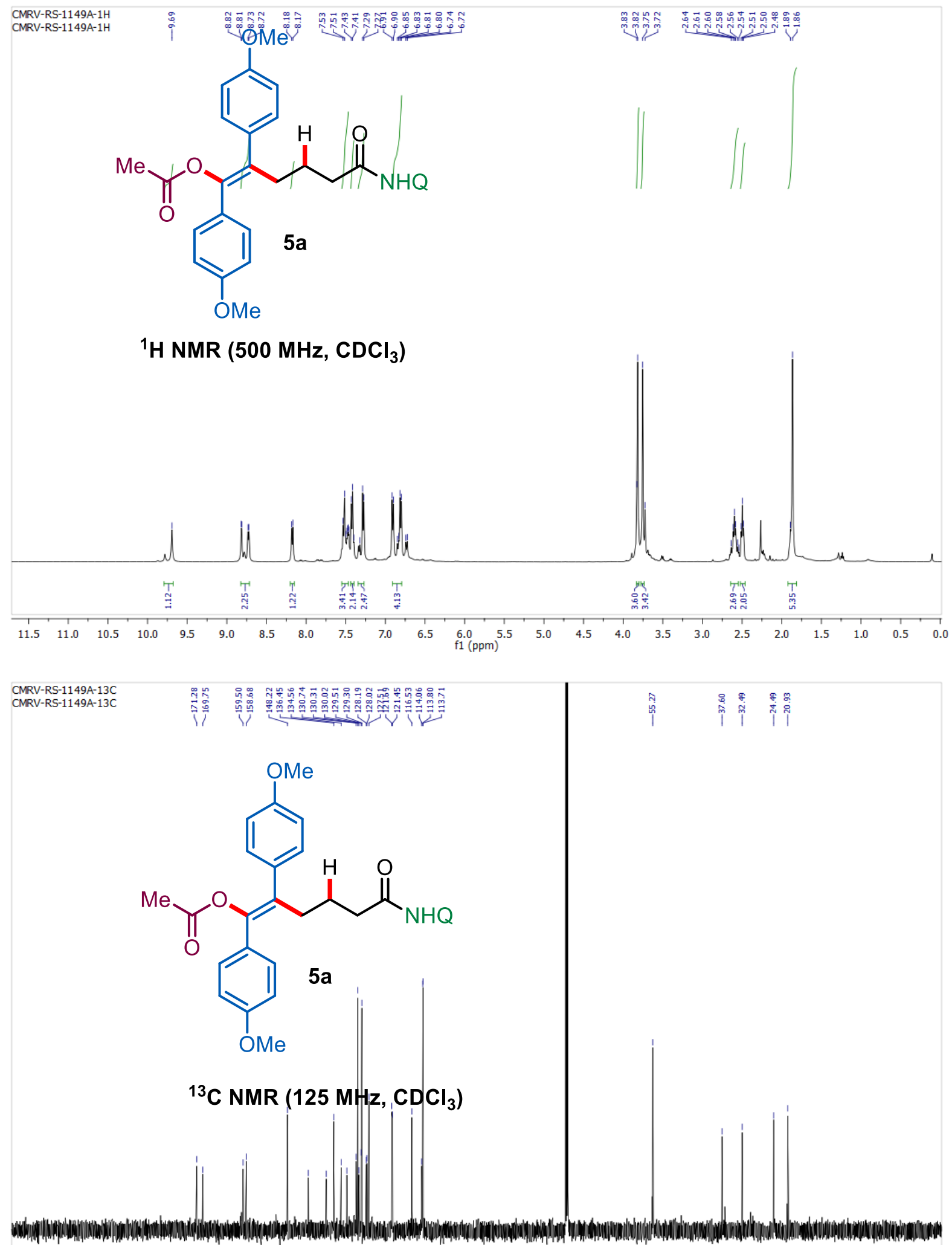

$\begin{array}{llllllllllllllllllllllllll}210 & 200 & 190 & 180 & 170 & 160 & 150 & 140 & 130 & 120 & 110 & 100 & 90 & 80 & 70 & 60 & 50 & 40 & 30 & 20 & 10 & 0 & -10\end{array}$ 

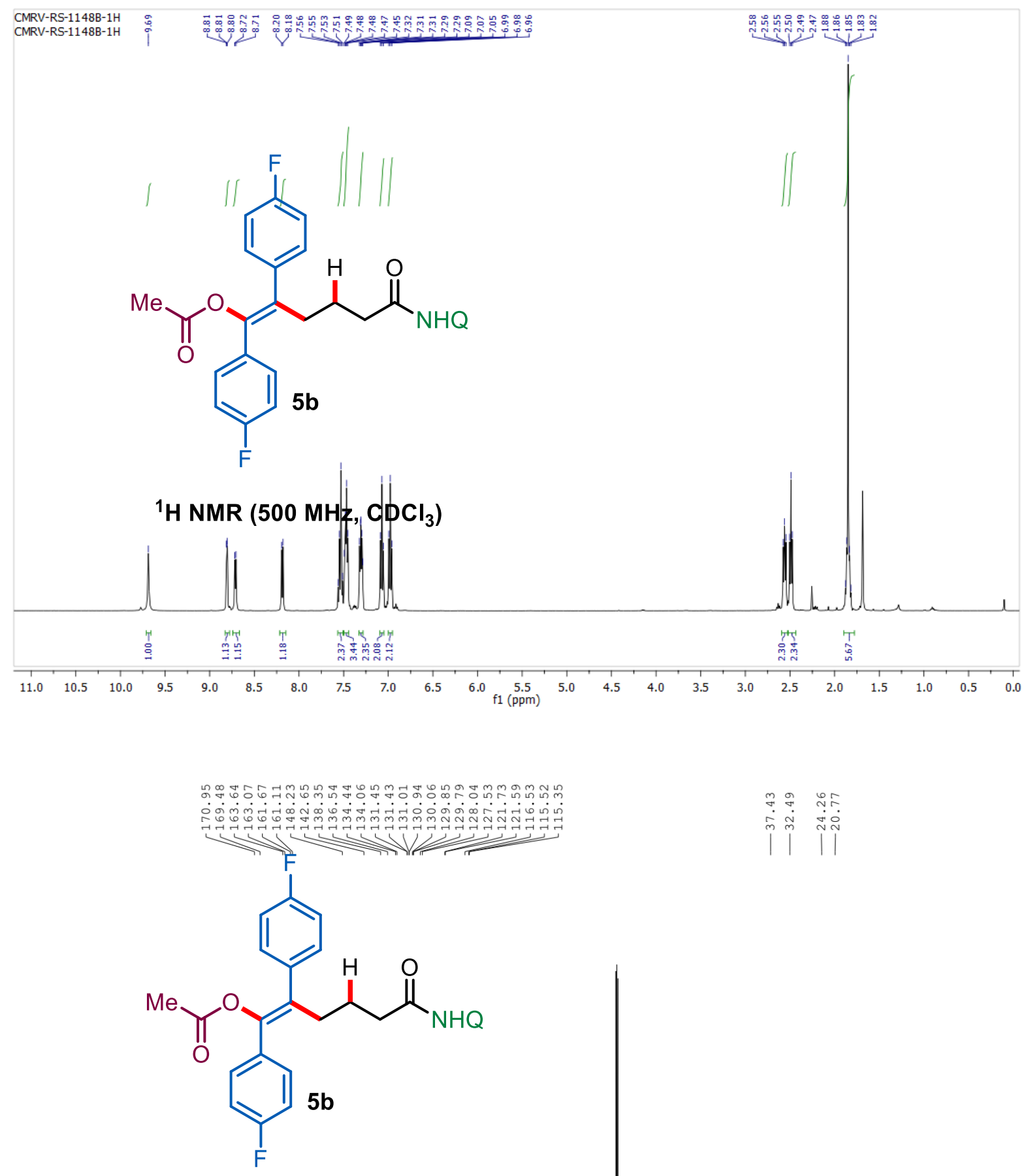

|

${ }^{13} \mathrm{C}$ NMR (125 $\left.\mathrm{MHz}, \mathrm{CDCl}_{3}\right)$

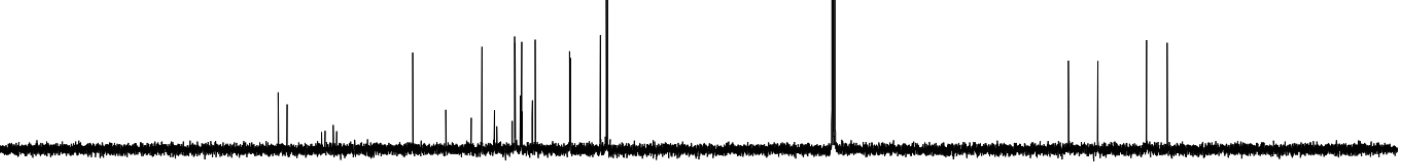

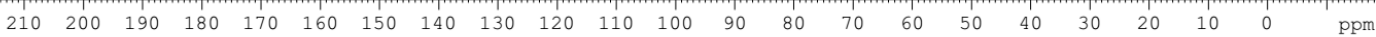



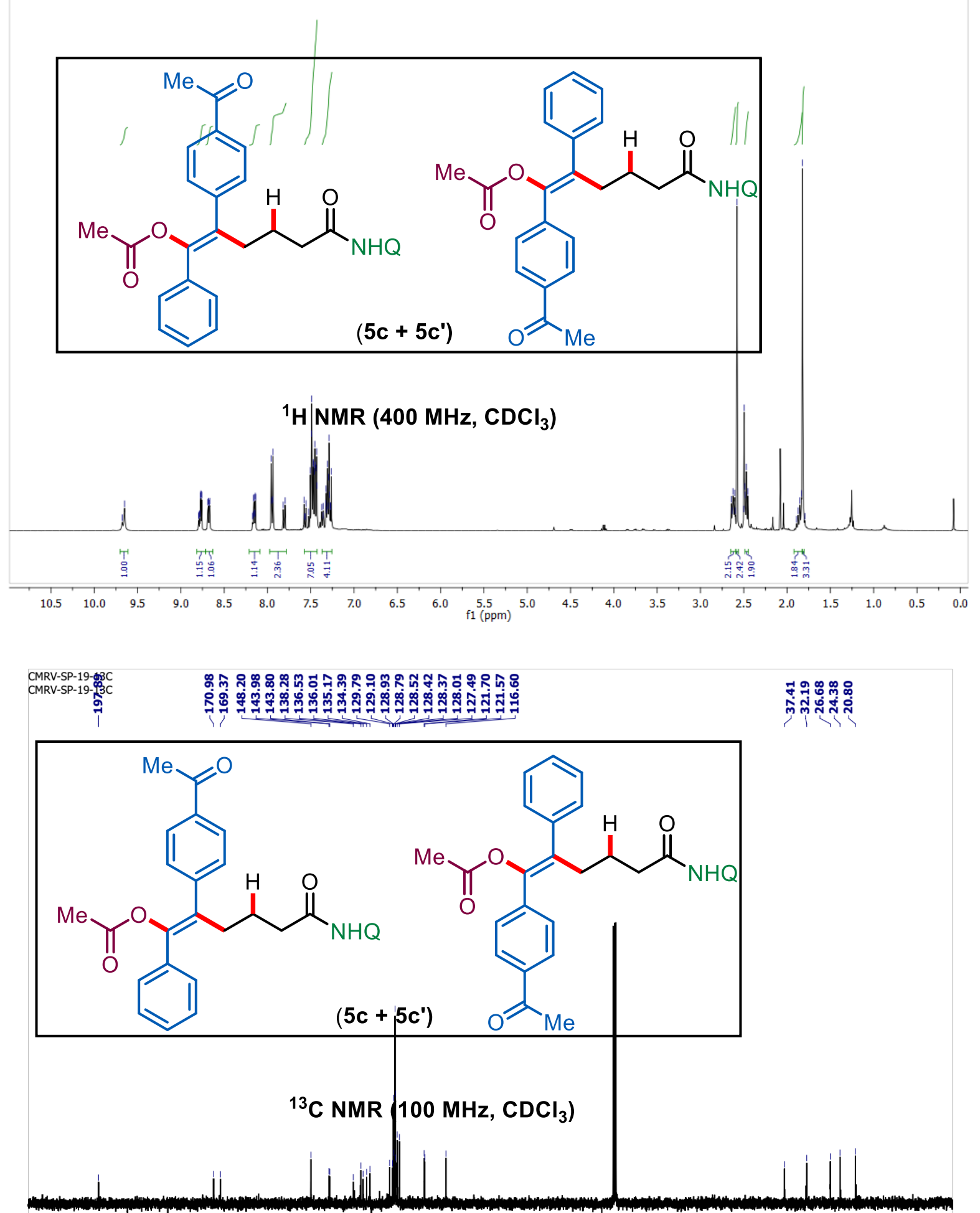

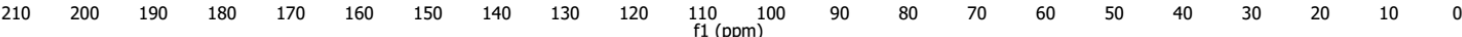



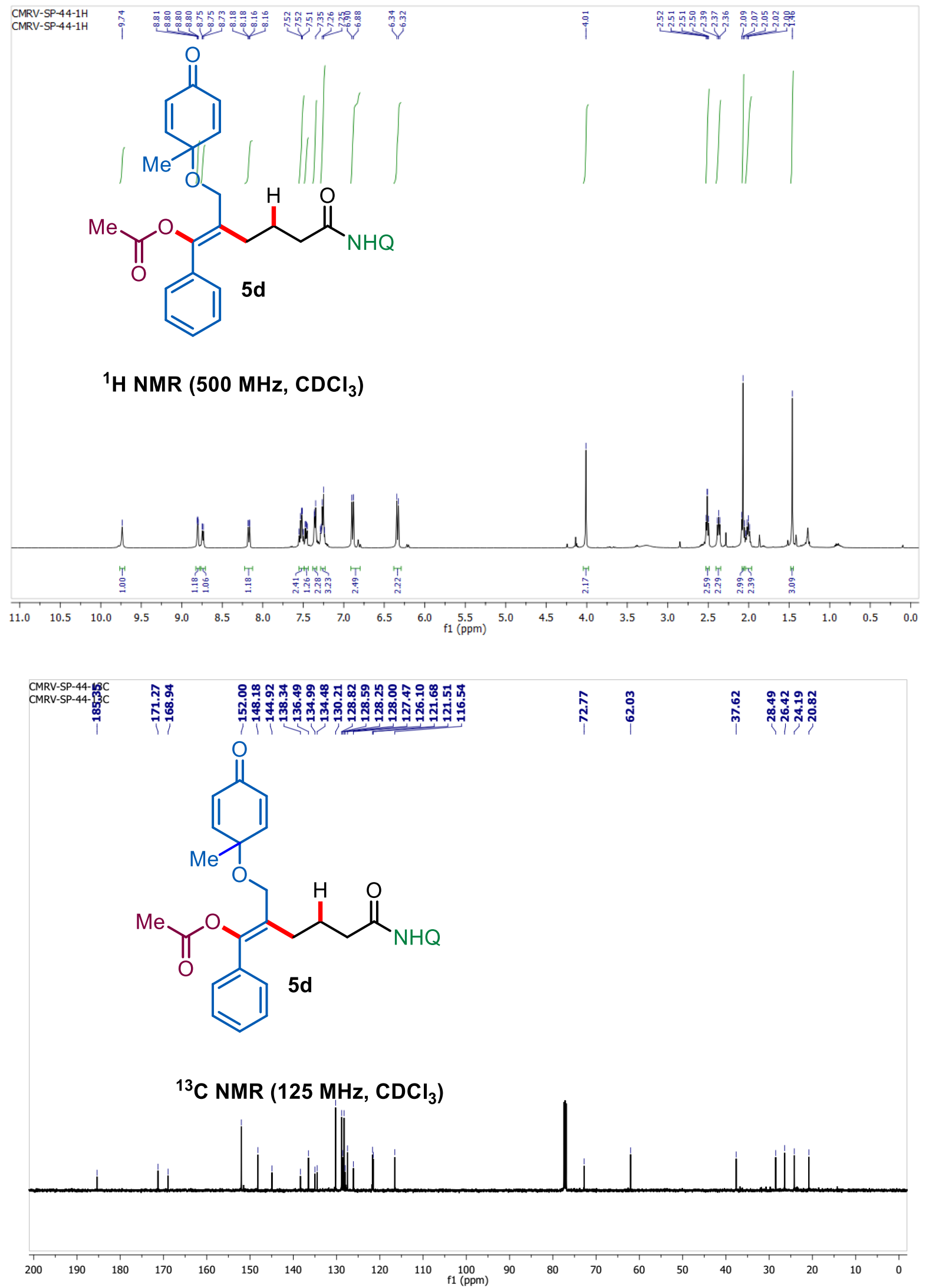

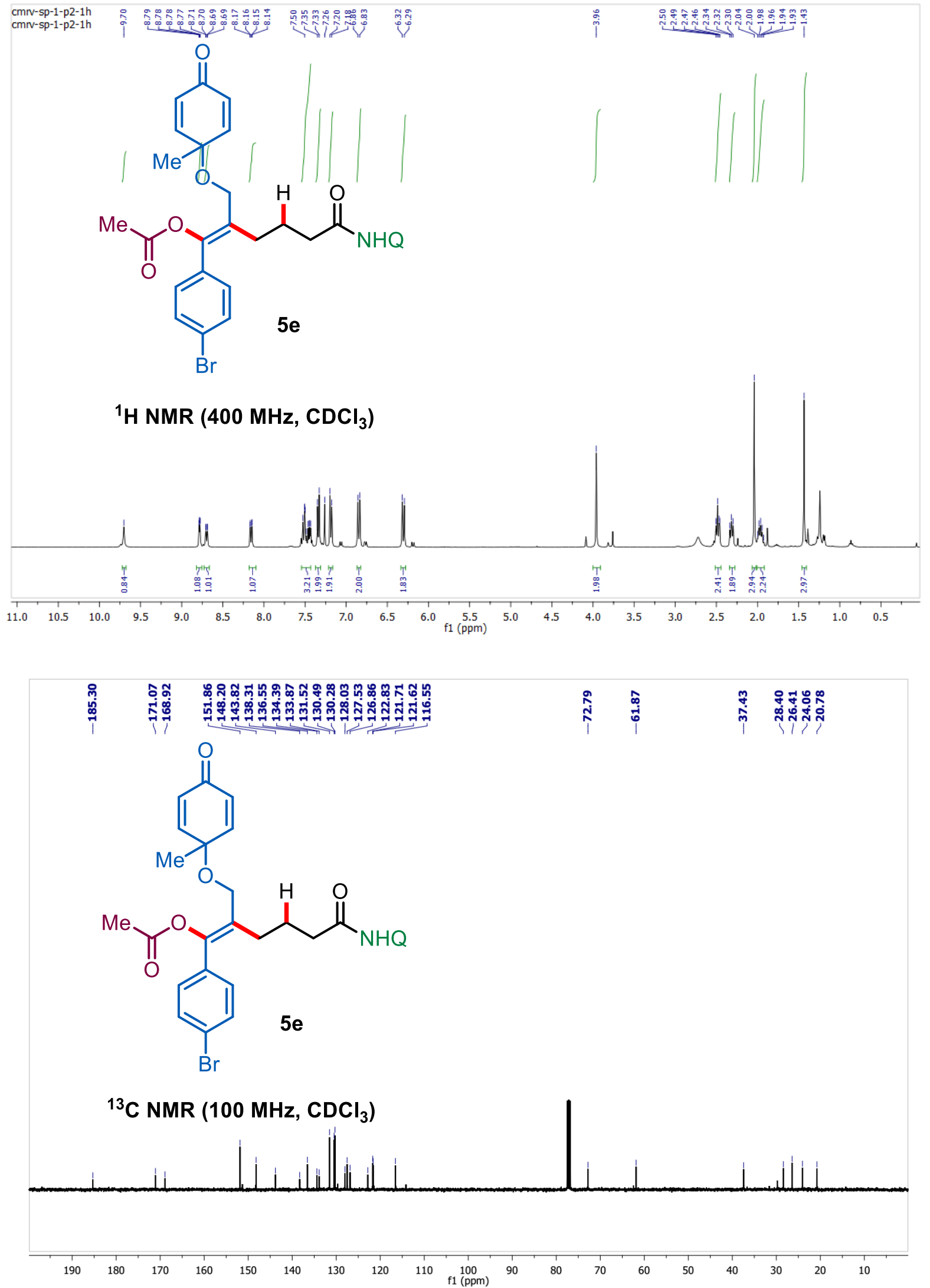


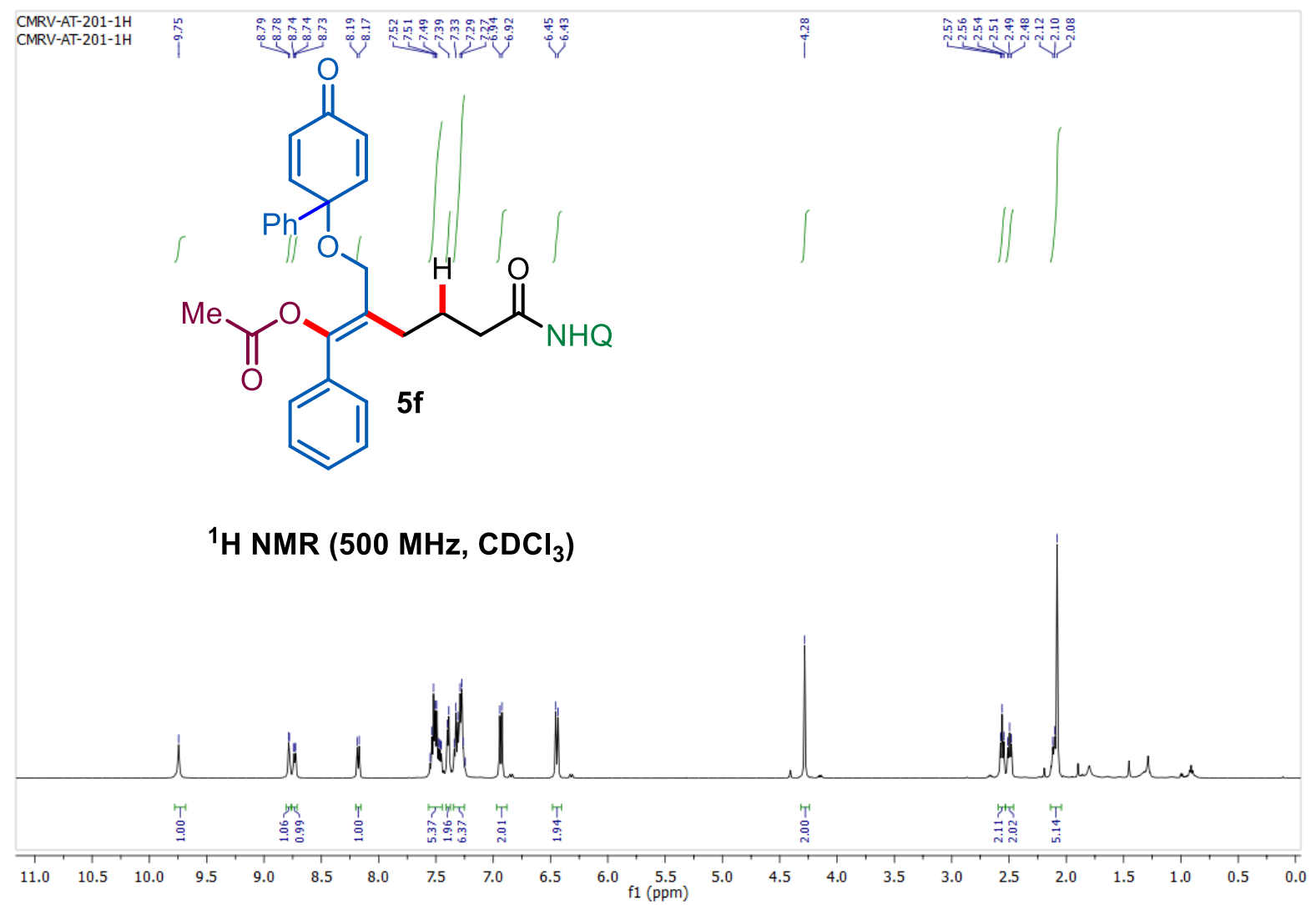

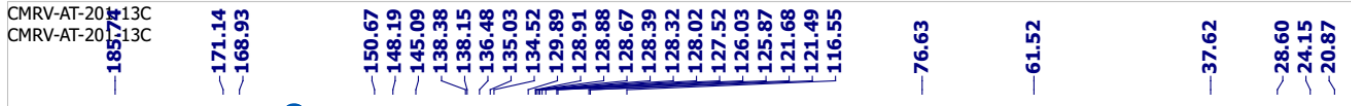<smiles>CC(=O)O/C(=C(/COC1(c2ccccc2)C=CC(=O)C=C1)CC(Br)CC(=O)NO)c1ccccc1</smiles>

${ }^{13} \mathrm{C}$ NMR (125 MHz, $\left.\mathrm{CDCl}_{3}\right)$

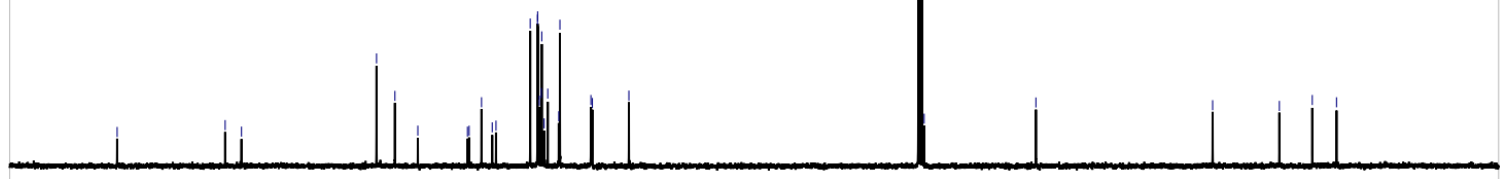

$\begin{array}{llllllllllllllllllllll}200 & 190 & 180 & 170 & 160 & 150 & 140 & 130 & 120 & 110 & 100 & 90 & 80 & 70 & 60 & 50 & 40 & 30 & 20 & 10 & 0\end{array}$ 


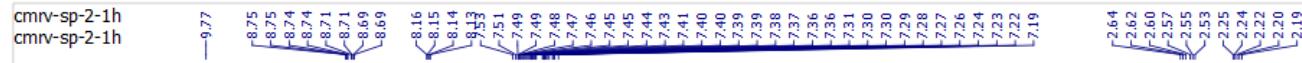<smiles>C#CCC(CC(=O)NO)CC(C#Cc1ccccc1)=C(OC(C)=O)c1ccccc1</smiles>

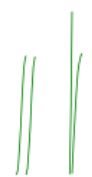

${ }^{1} \mathrm{H}$ NMR (400 MHz, $\left.\mathrm{CDCl}_{3}\right)$
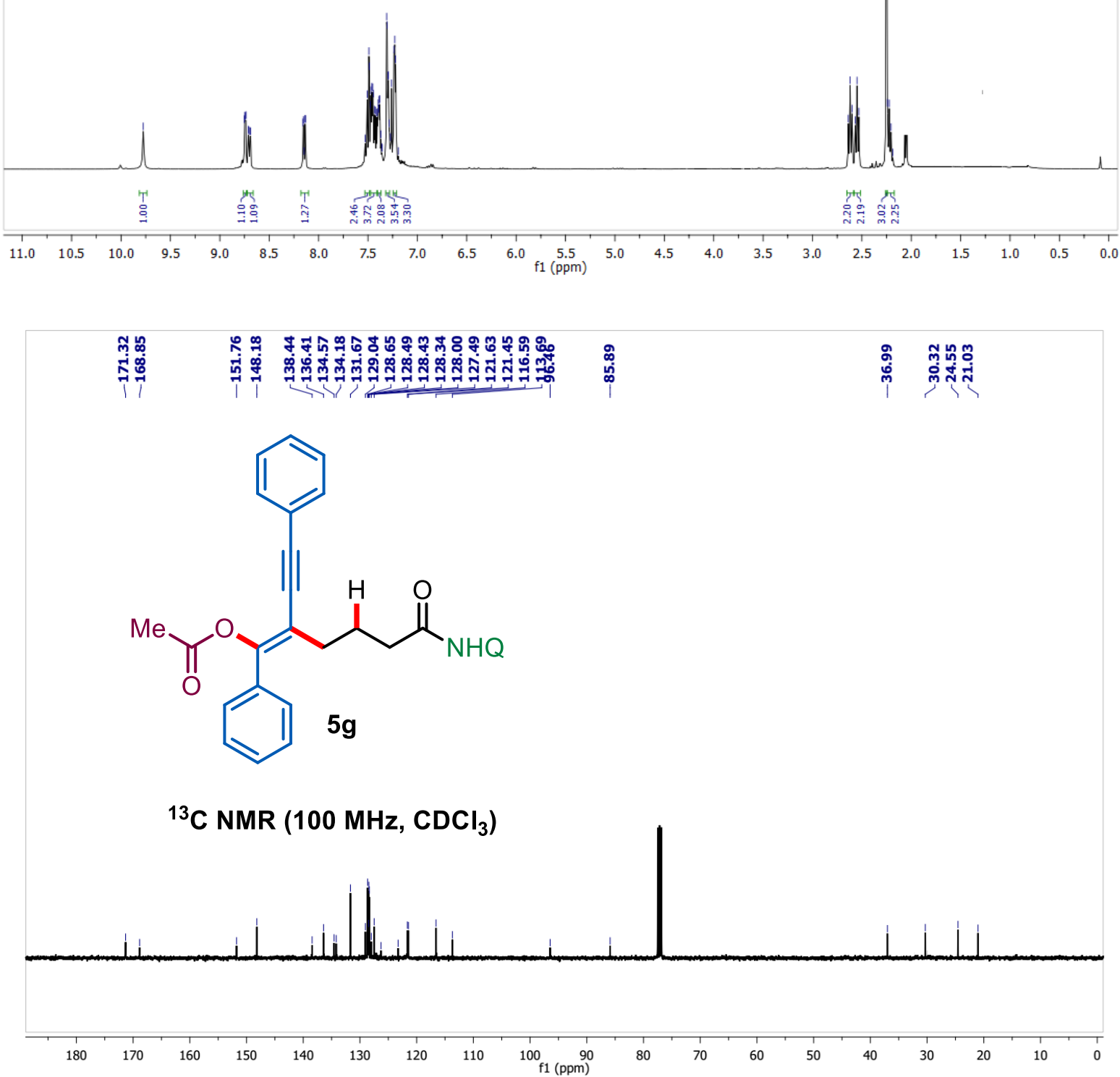

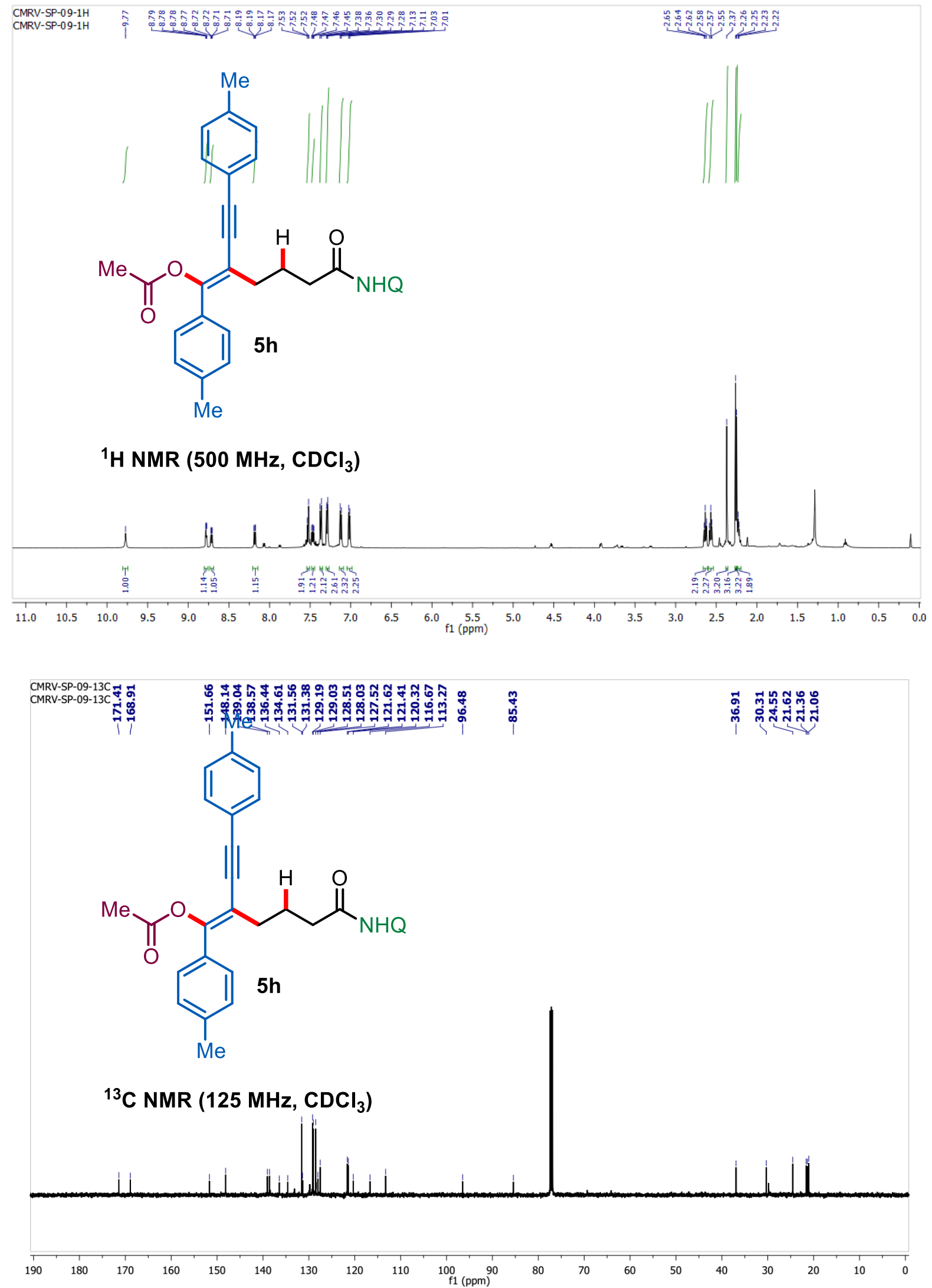
<smiles>CC#C/C(CC(C)CC(=O)NO)=C(/OC(C)=O)c1cccs1</smiles>
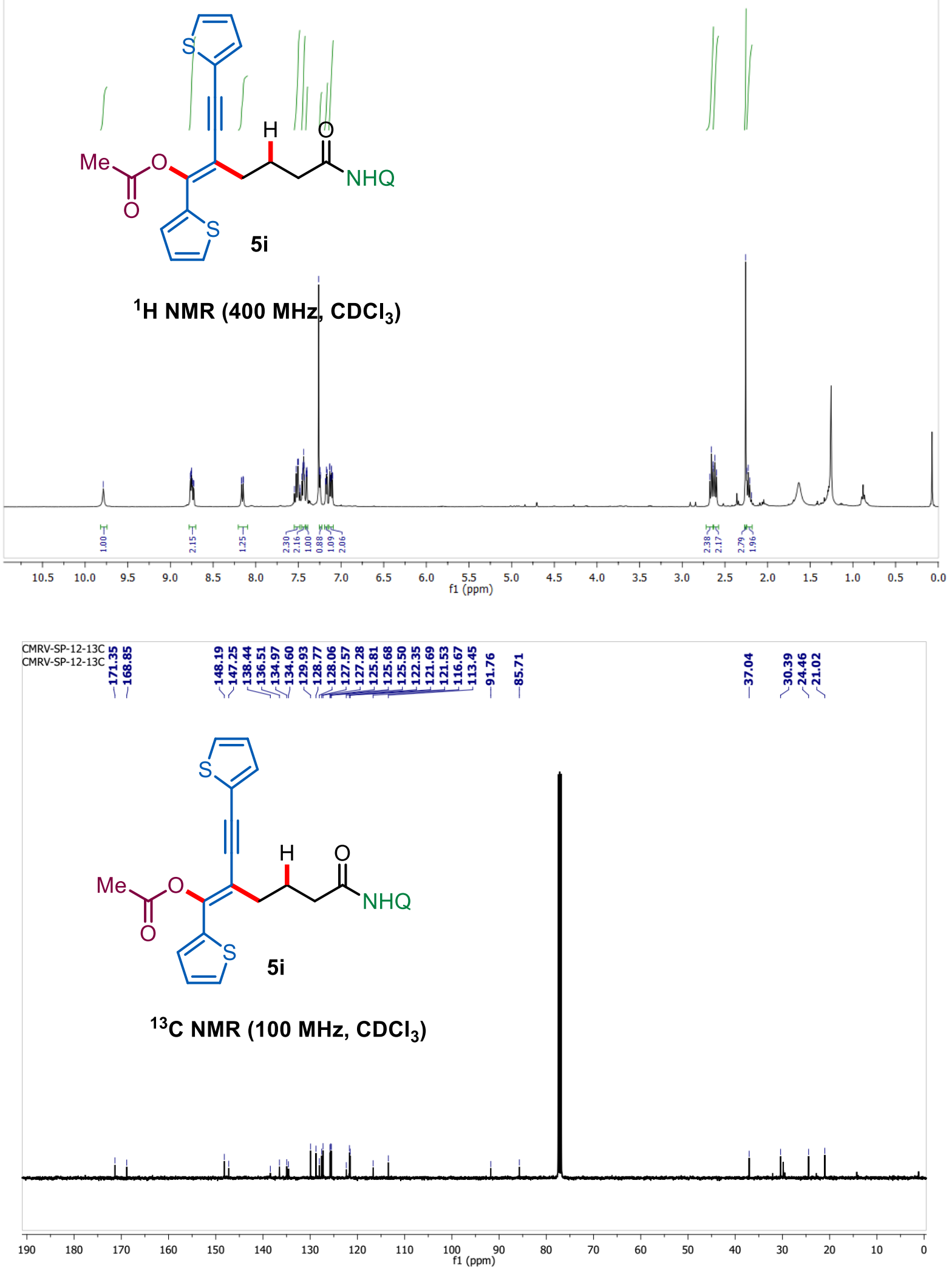

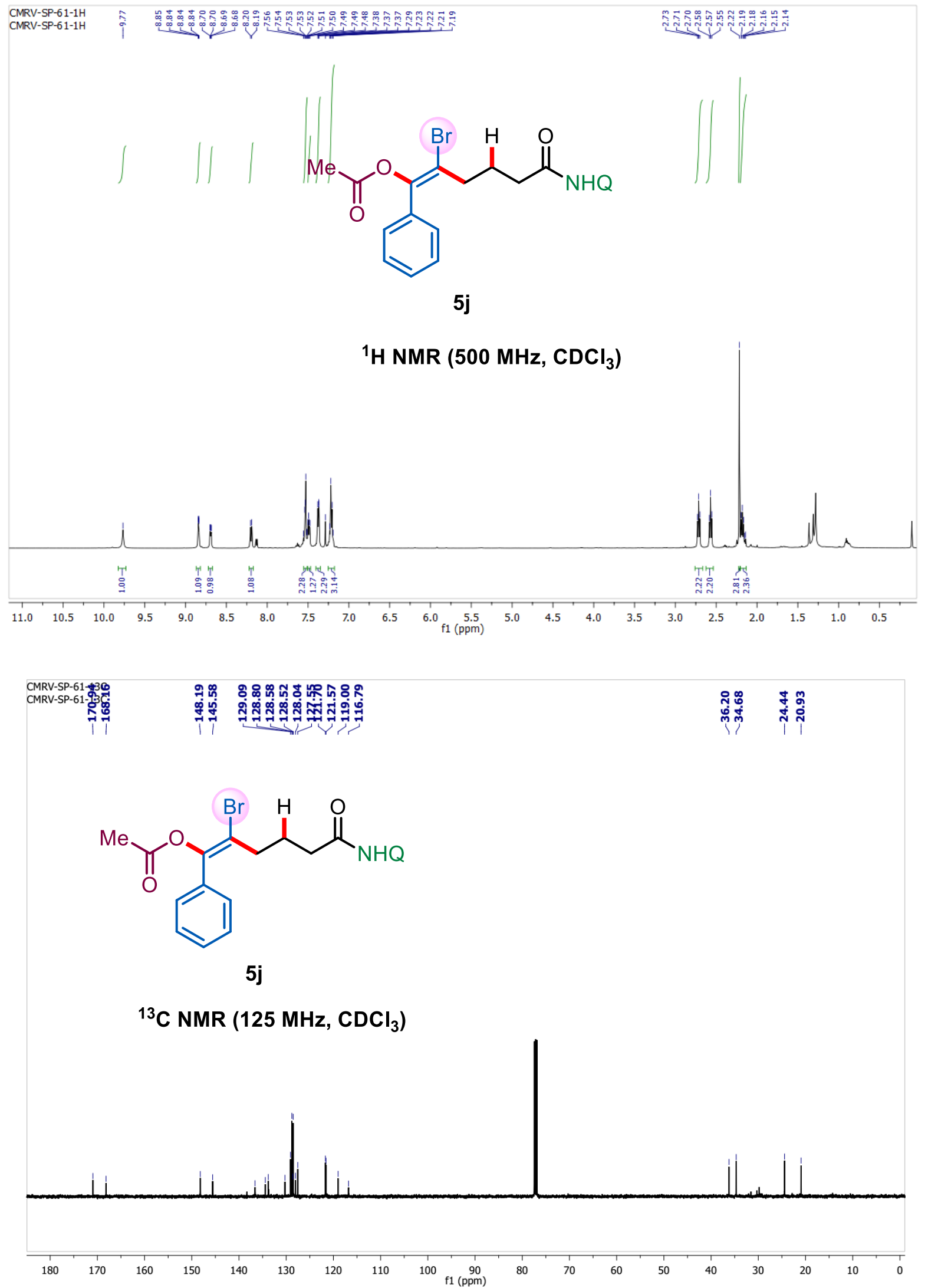

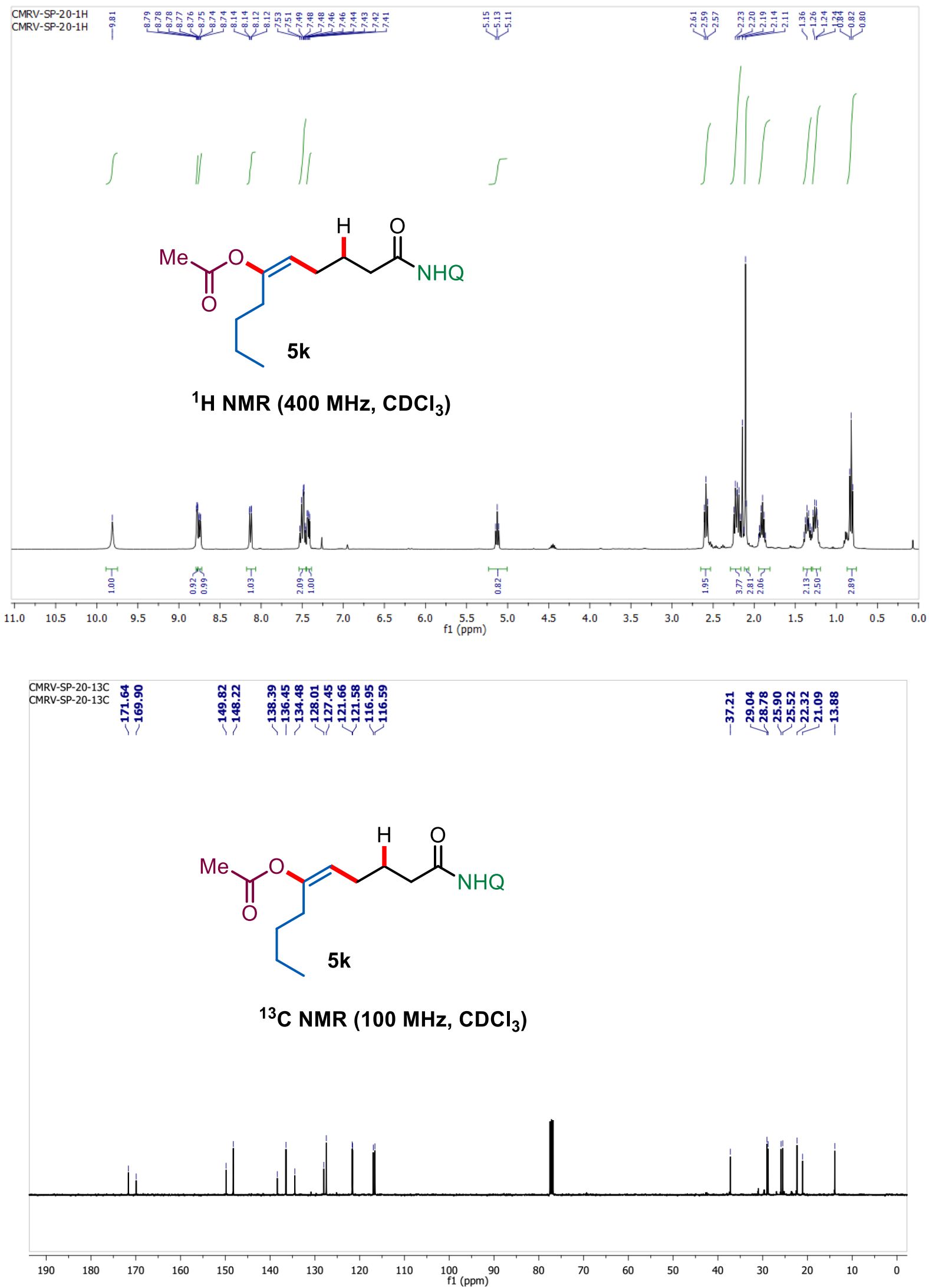

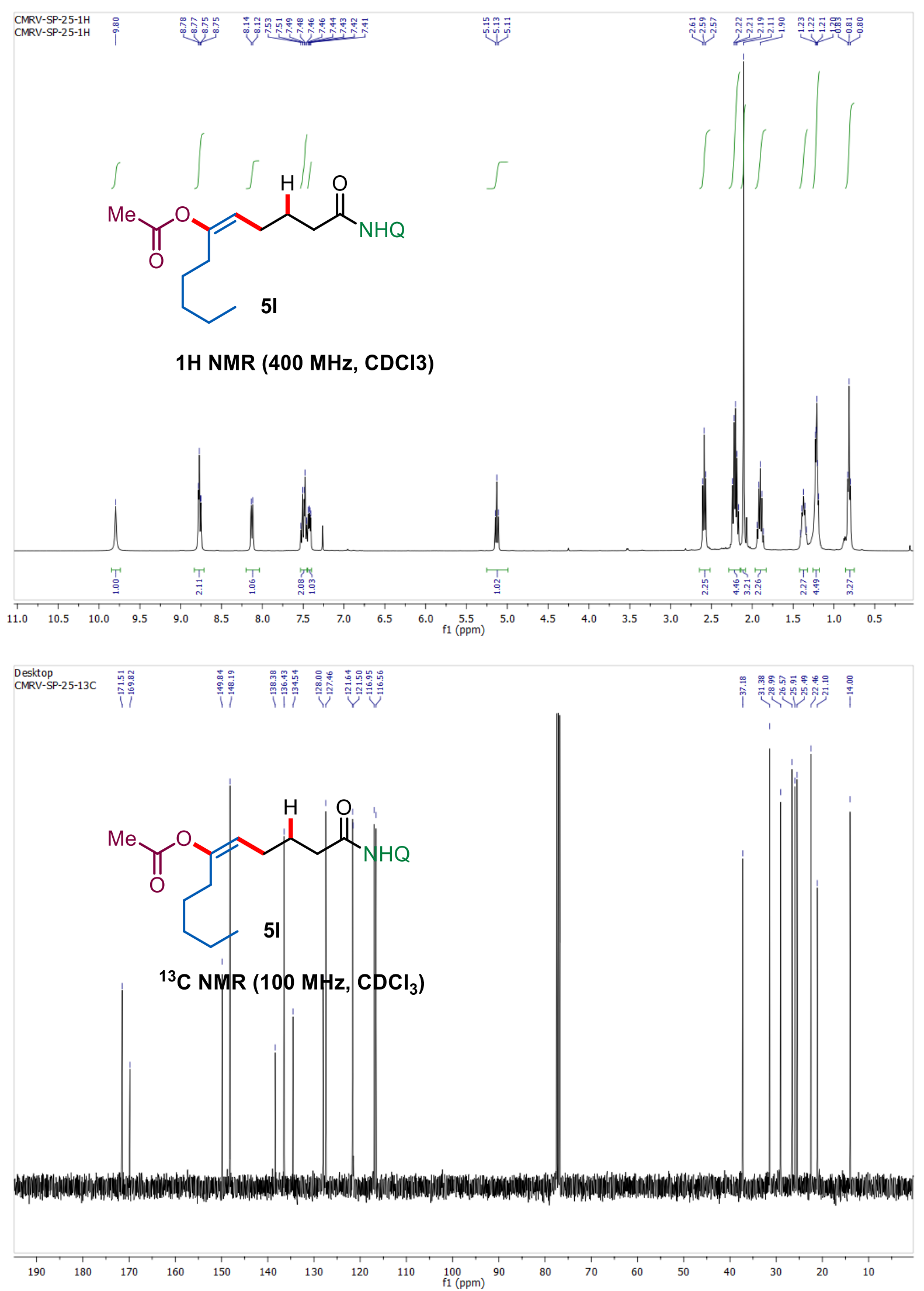

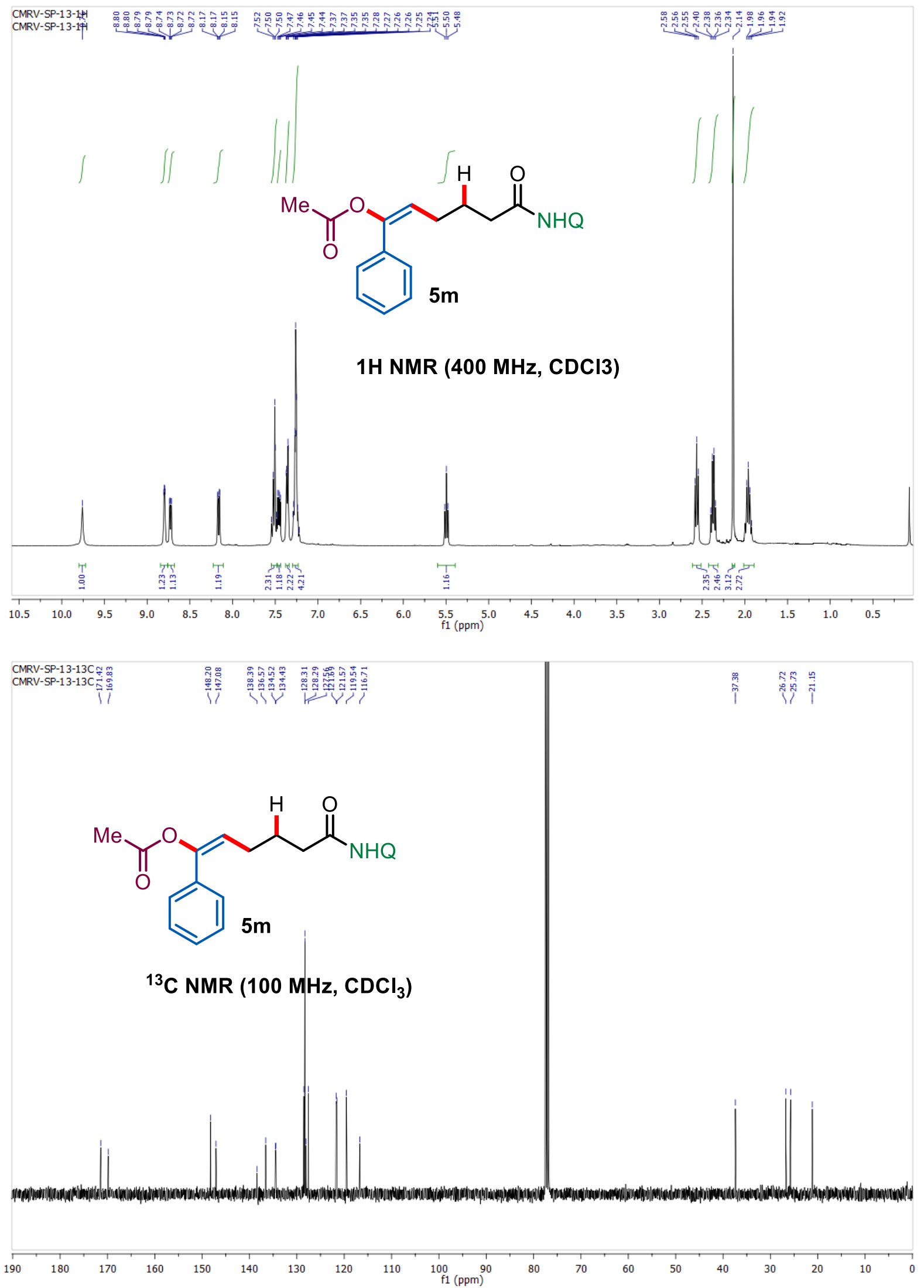

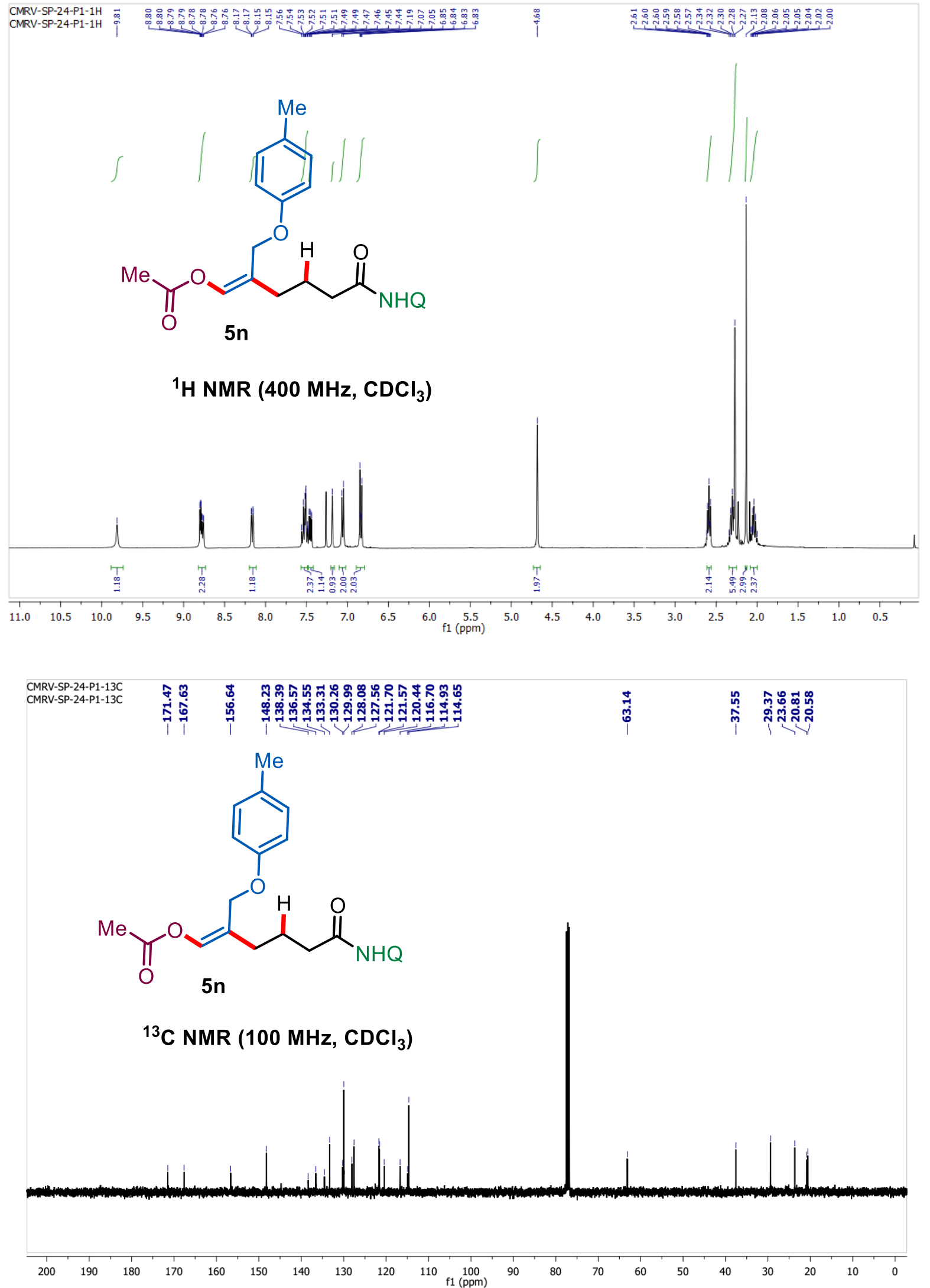

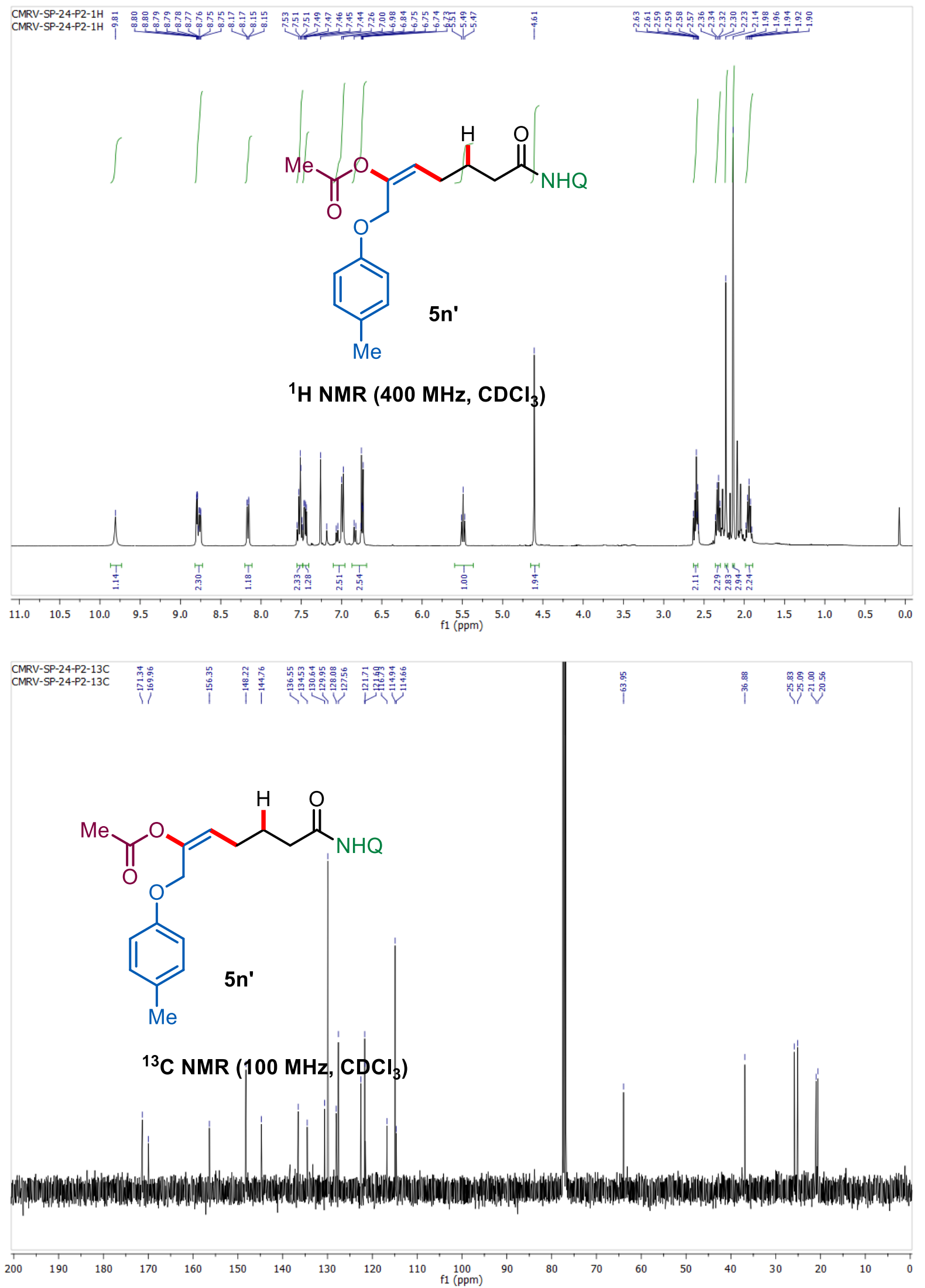


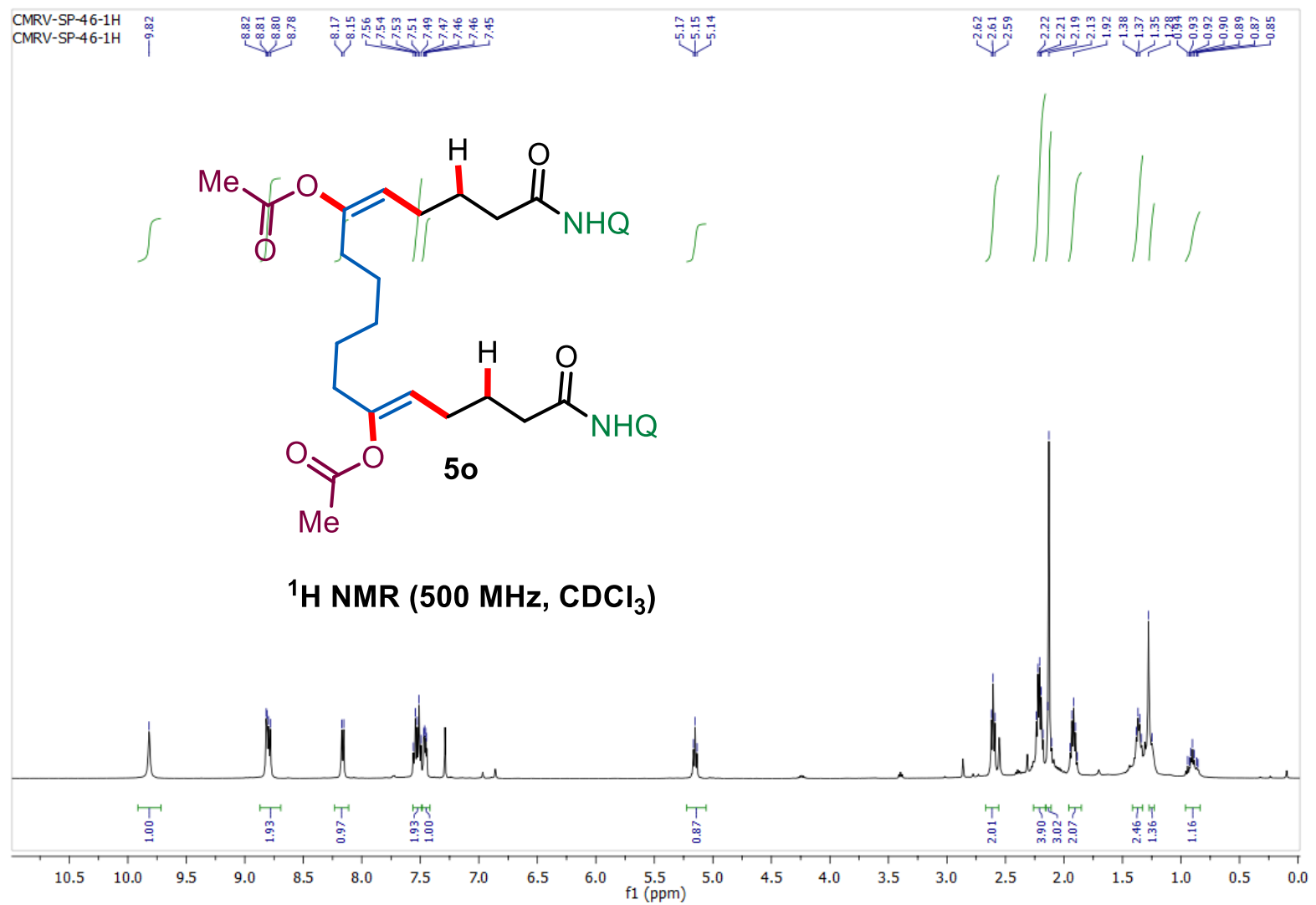

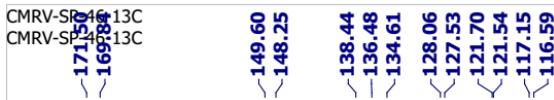

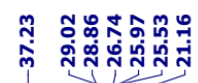

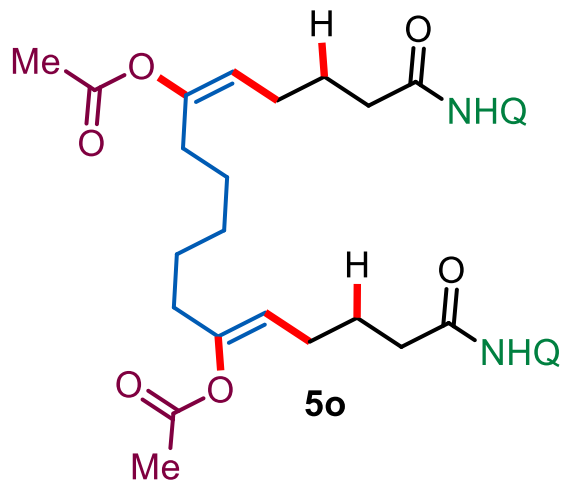

${ }^{13} \mathrm{C}$ NMR (125 MHz, $\left.\mathrm{CDCl}_{3}\right)$

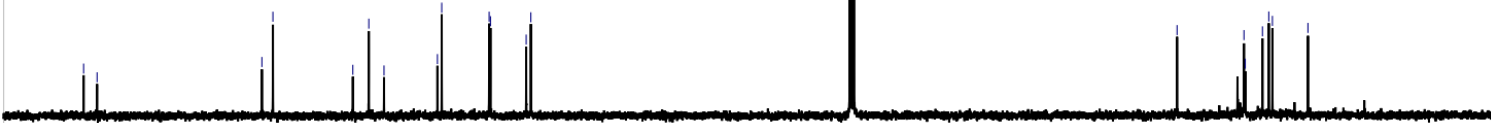

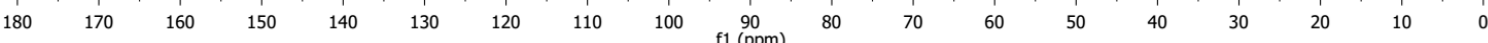



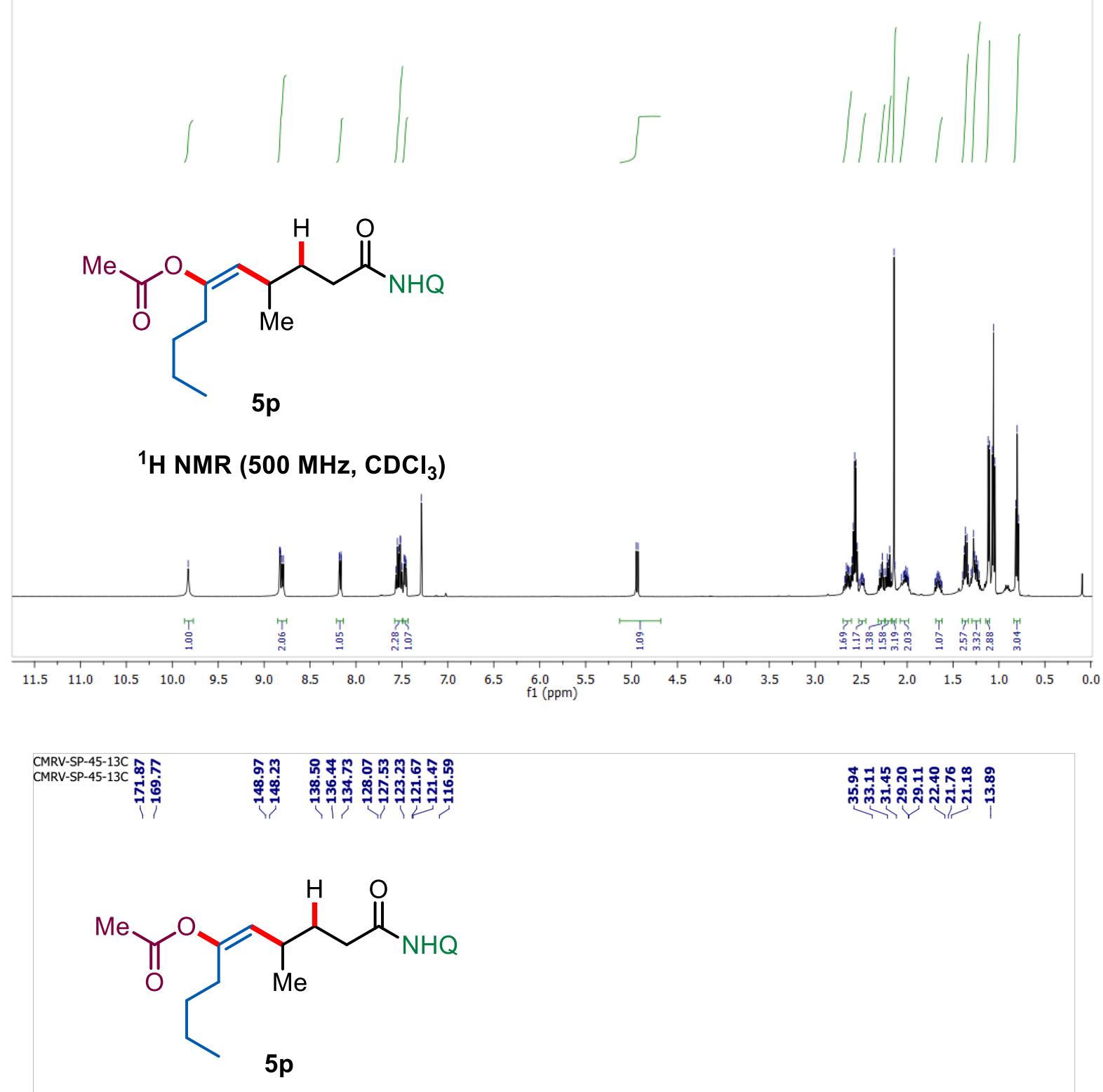

${ }^{13} \mathrm{C}$ NMR $\left(125 \mathrm{MHz}, \mathrm{CDCl}_{3}\right)$

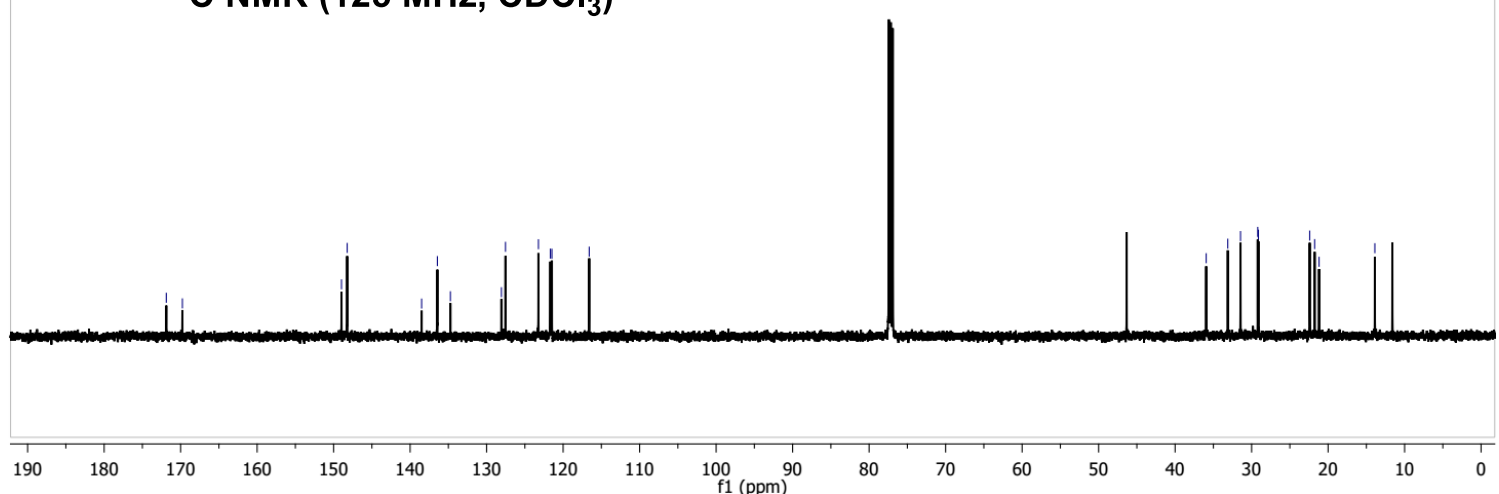


<smiles>CCCC/C(=C\C(CC)CC(=O)NO)OC(C)=O</smiles>

$5 q$

${ }^{1} \mathrm{H}$ NMR (400 MHz, $\left.\mathrm{CDCl}_{3}\right)$<smiles>CCCC/C(=C\C(CC)CCC(=O)NO)OC(C)=O</smiles>

$5 q$

${ }^{13} \mathrm{C}$ NMR $\left(125 \mathrm{MHz}, \mathrm{CDCl}_{3}\right)$

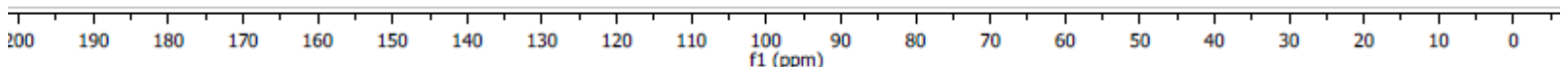




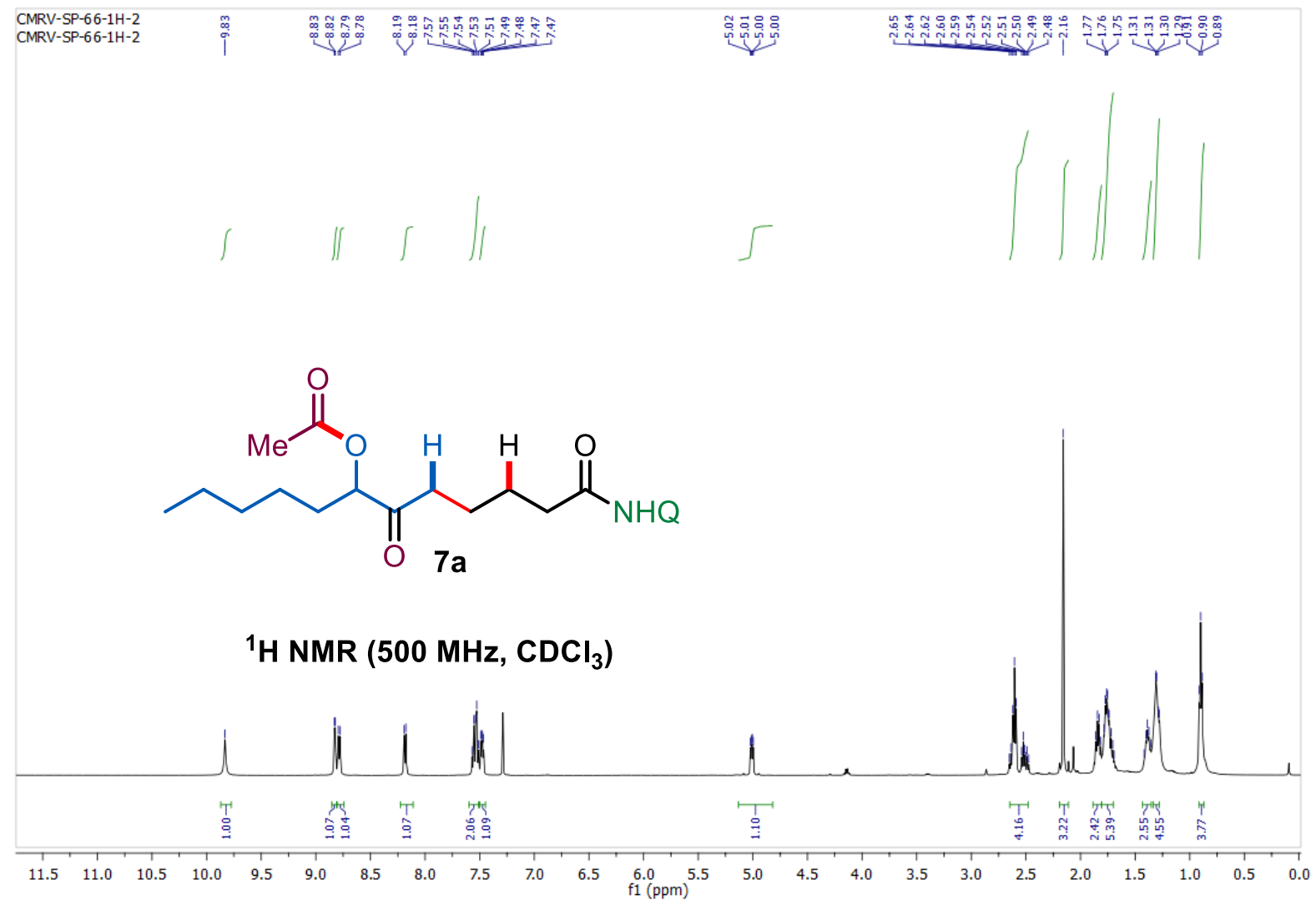

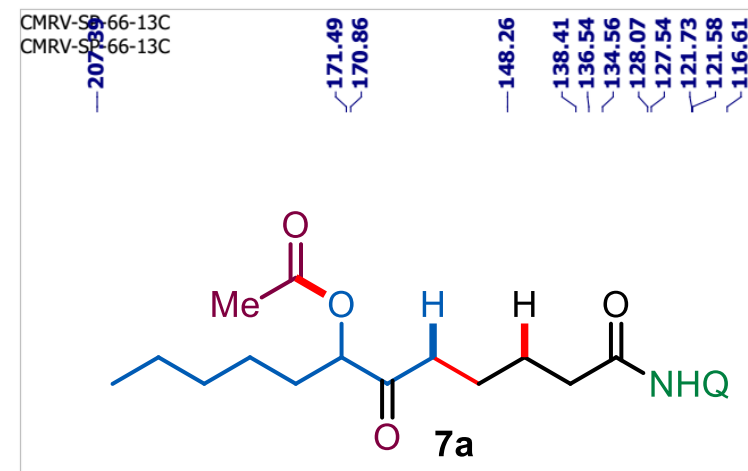

${ }^{1} \mathrm{H}$ NMR $\left(125 \mathrm{MHz}, \mathrm{CDCl}_{3}\right)$

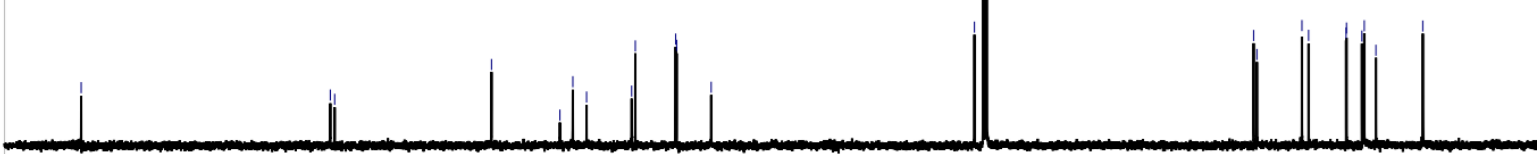

$\begin{array}{llllllllllllllllllllll}210 & 200 & 190 & 180 & 170 & 160 & 150 & 140 & 130 & 120 & 110 & 100 & 90 & 80 & 70 & 60 & 50 & 40 & 30 & 20 & 10 & 0\end{array}$ 


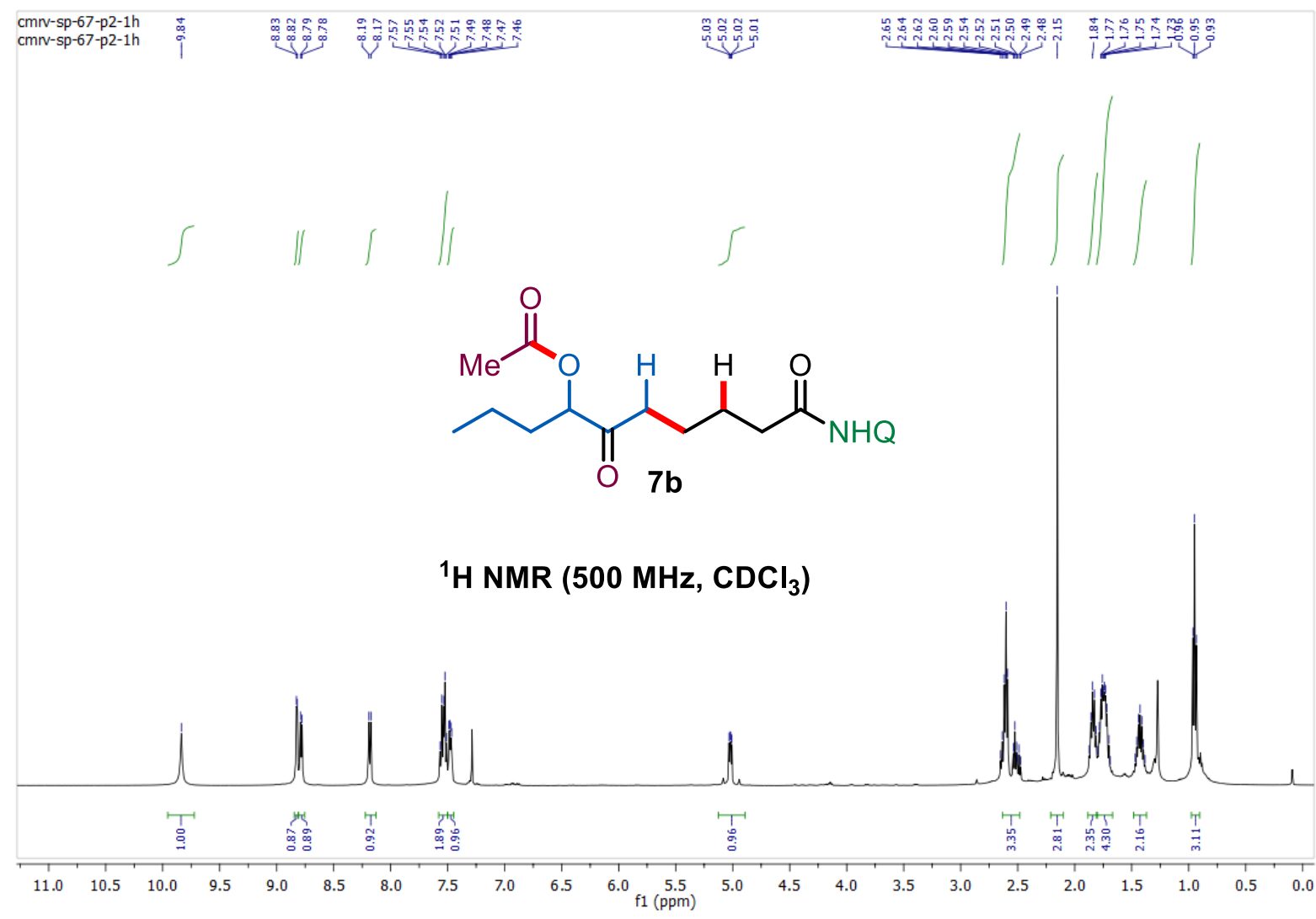

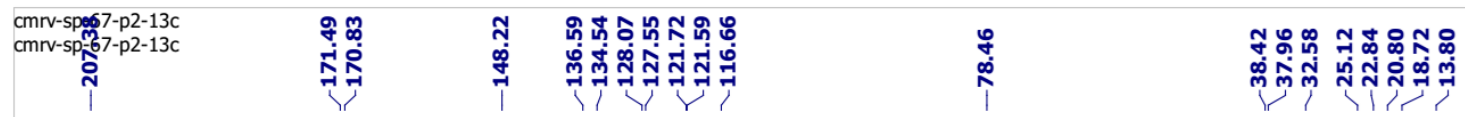<smiles>CCCC(OC(C)=O)C(=O)CCCC(=O)NO</smiles>

${ }^{13} \mathrm{C}$ NMR (125 MHz, $\left.\mathrm{CDCl}_{3}\right)$

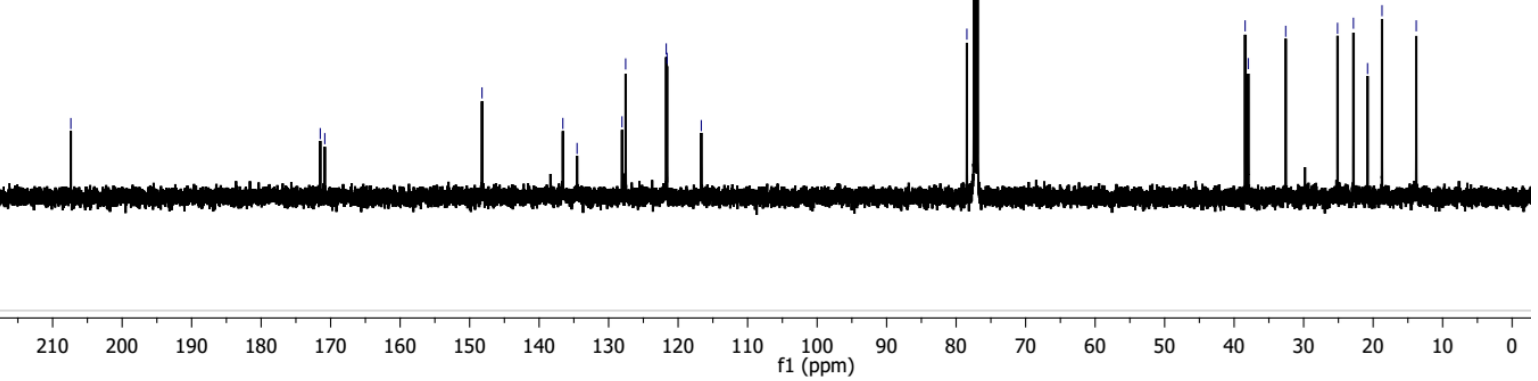


<smiles>CCCCC(CC)C(OC(C)=O)C(=O)CCCC(=O)NO</smiles>

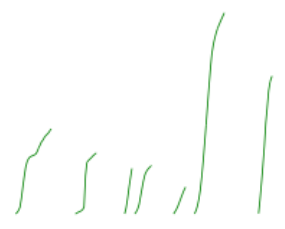

${ }^{1} \mathrm{H}$ NMR (500 MHz, $\left.\mathrm{CDCl}_{3}\right)$
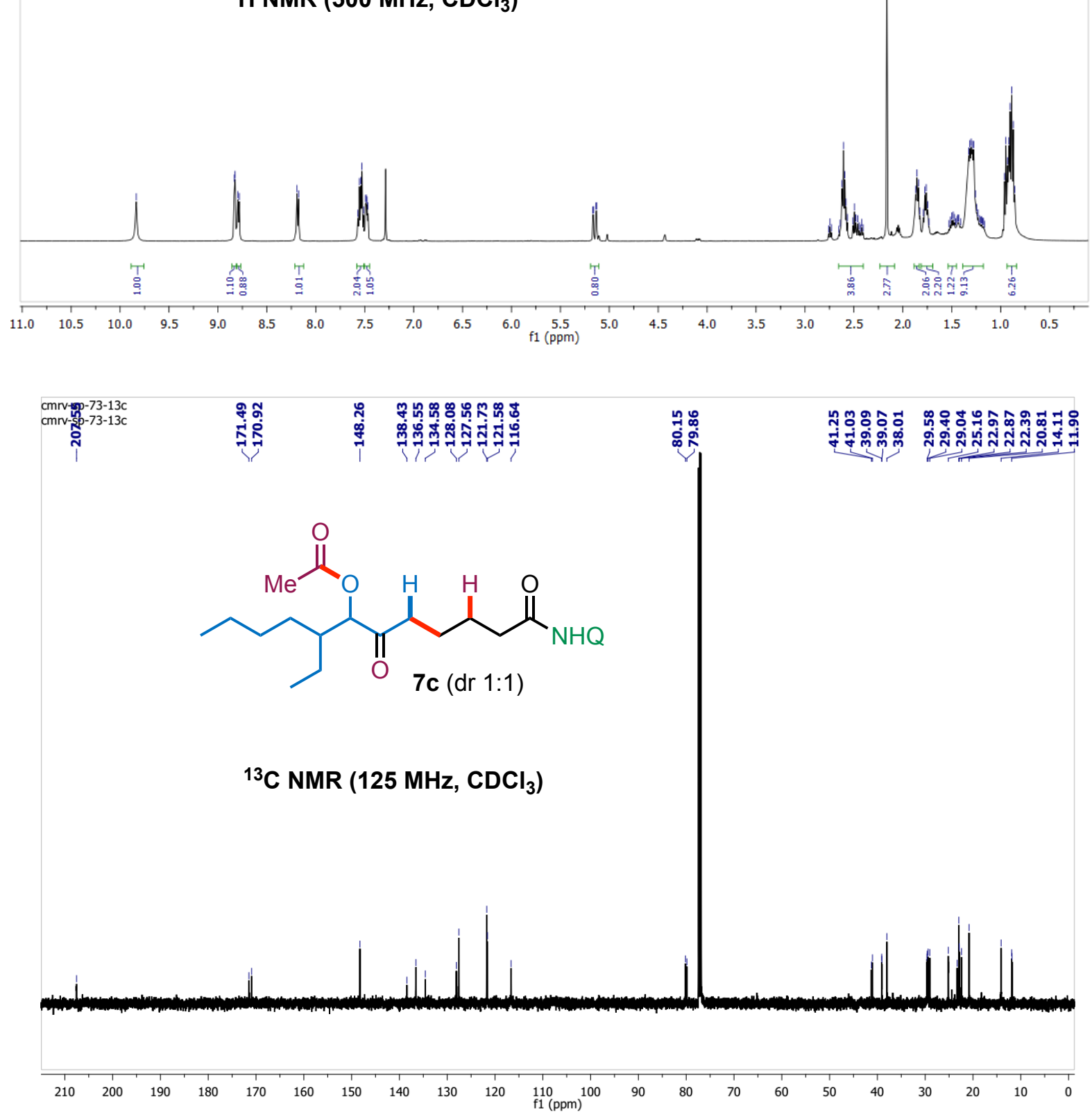

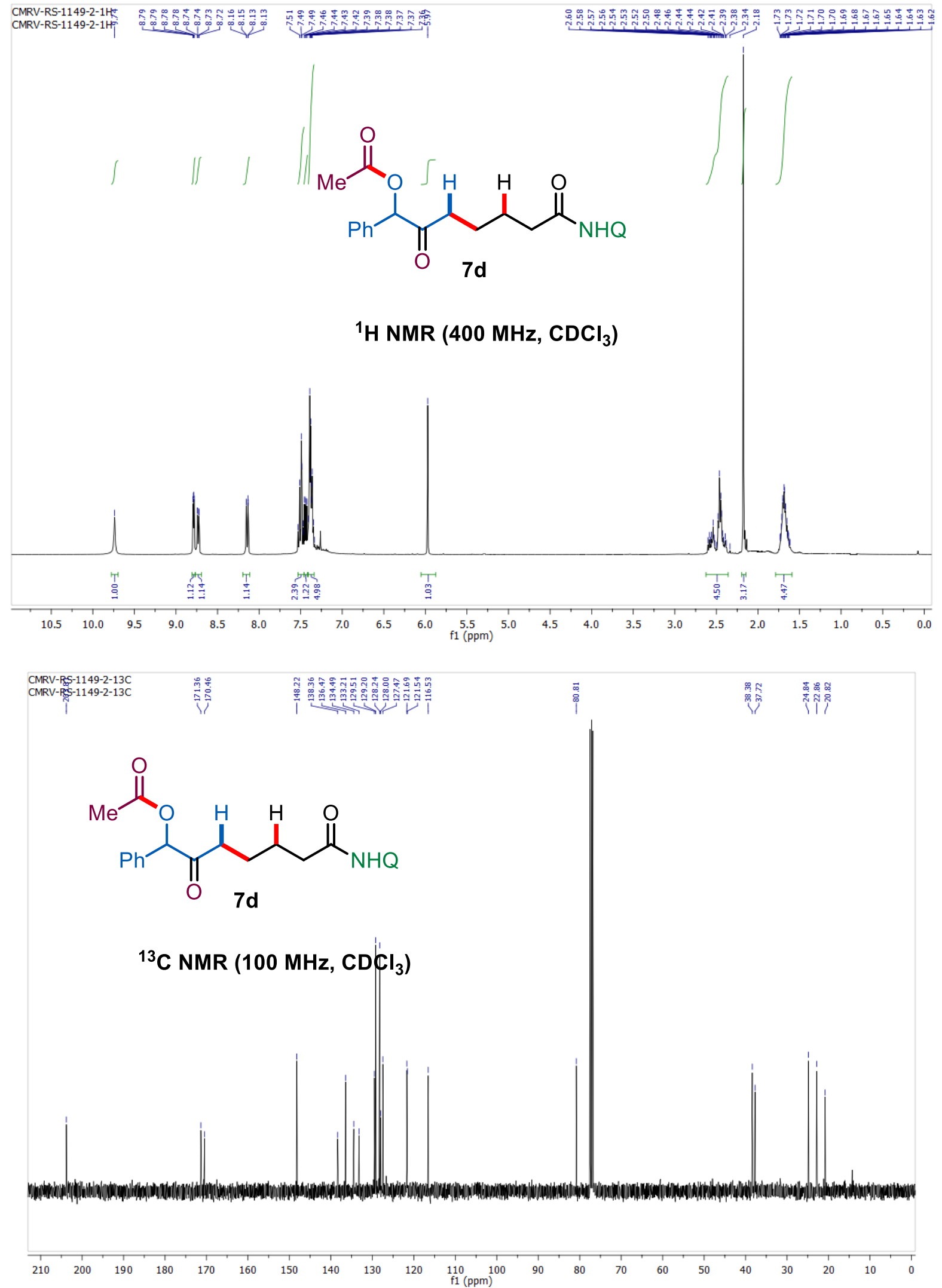


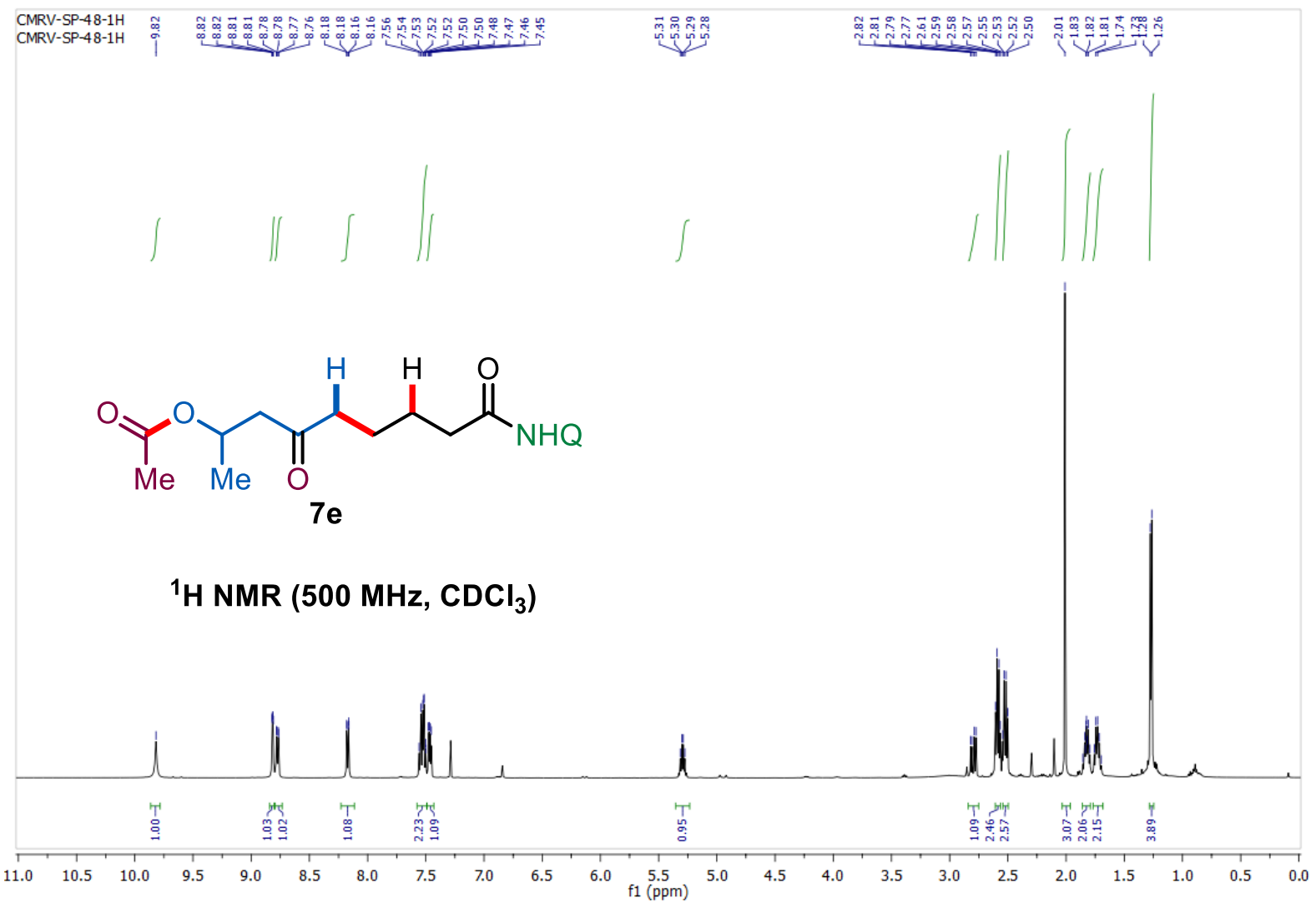

\begin{tabular}{|c|c|c|c|c|}
\hline 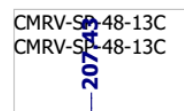 & 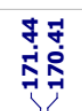 & 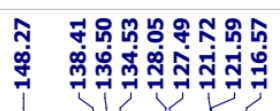 & ָ̦ & 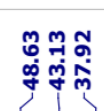 \\
\hline
\end{tabular}<smiles>CC(=O)OC(C)CC(=O)CCC=CCC(=O)NO</smiles>

${ }^{13} \mathrm{C}$ NMR $\left(125 \mathrm{MHz}, \mathrm{CDCl}_{3}\right)$

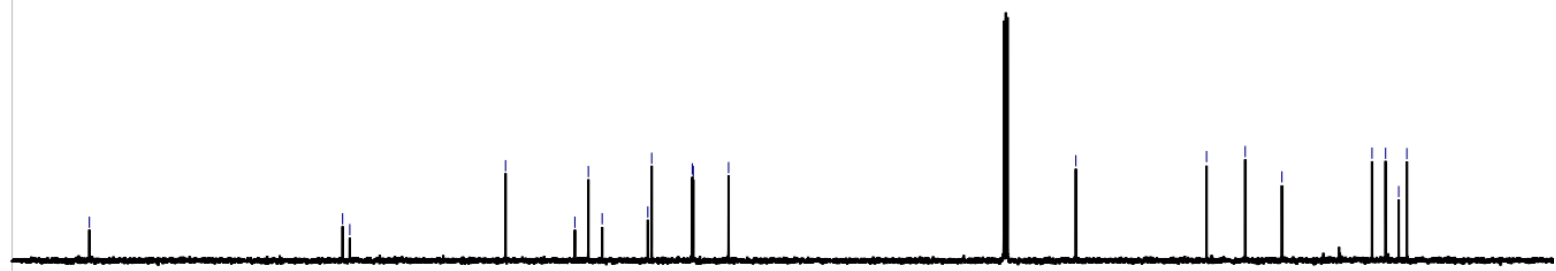

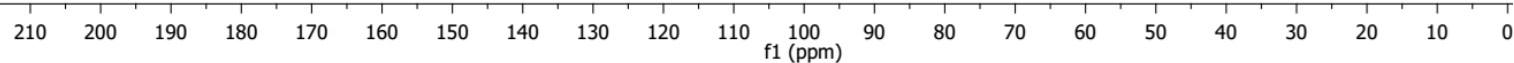



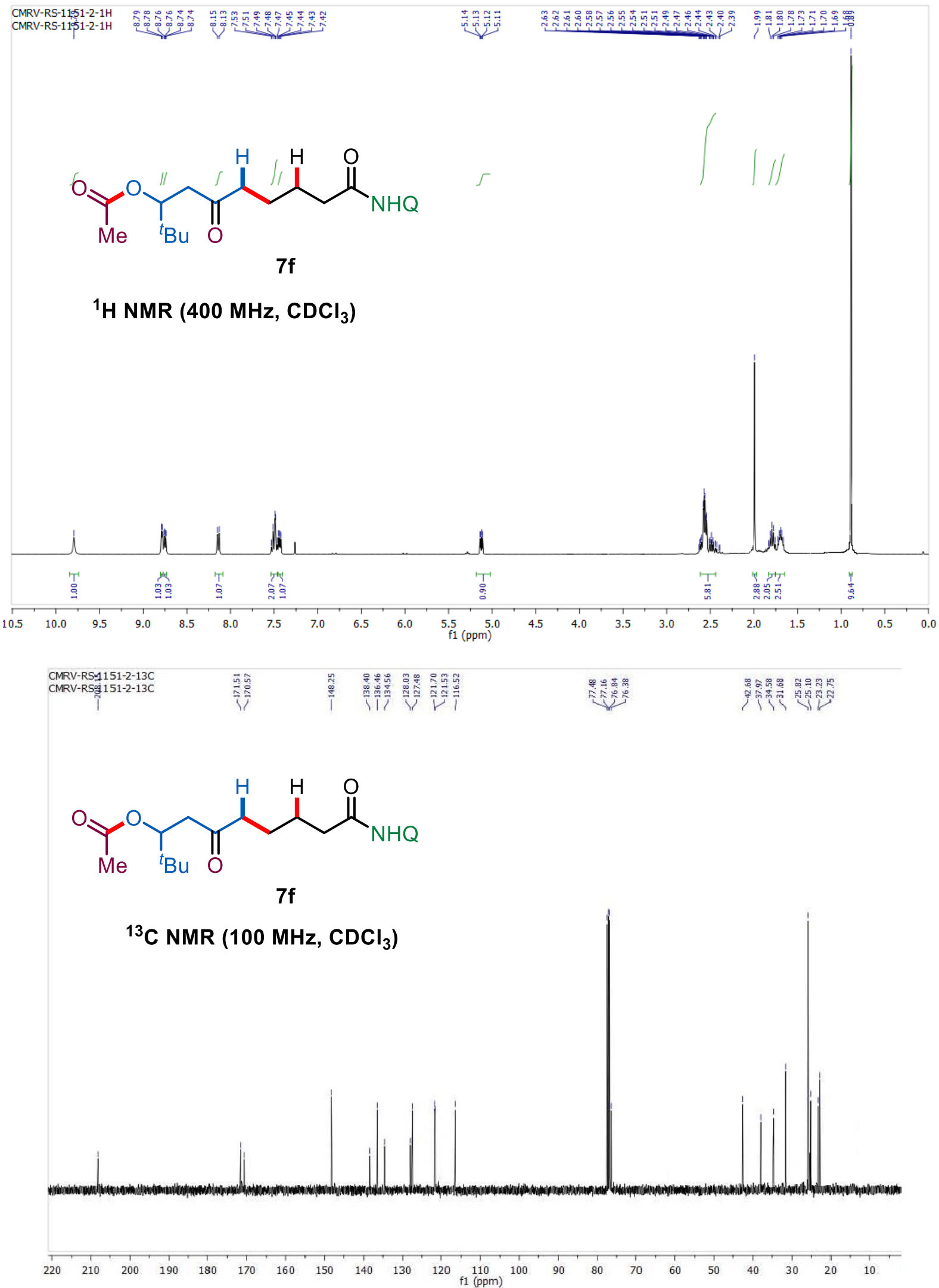
<smiles>CC(=O)O/C(CC(=O)/C=C/CC(=O)NO)=C(\C=C/[13CH3])C1CCCCC1</smiles>

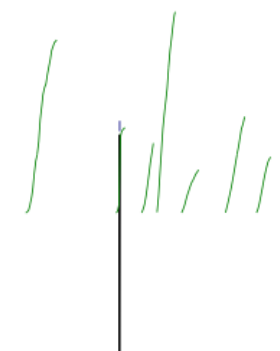

${ }^{1} \mathrm{H}$ NMR (400 MHz, $\left.\mathrm{CDCl}_{3}\right)$
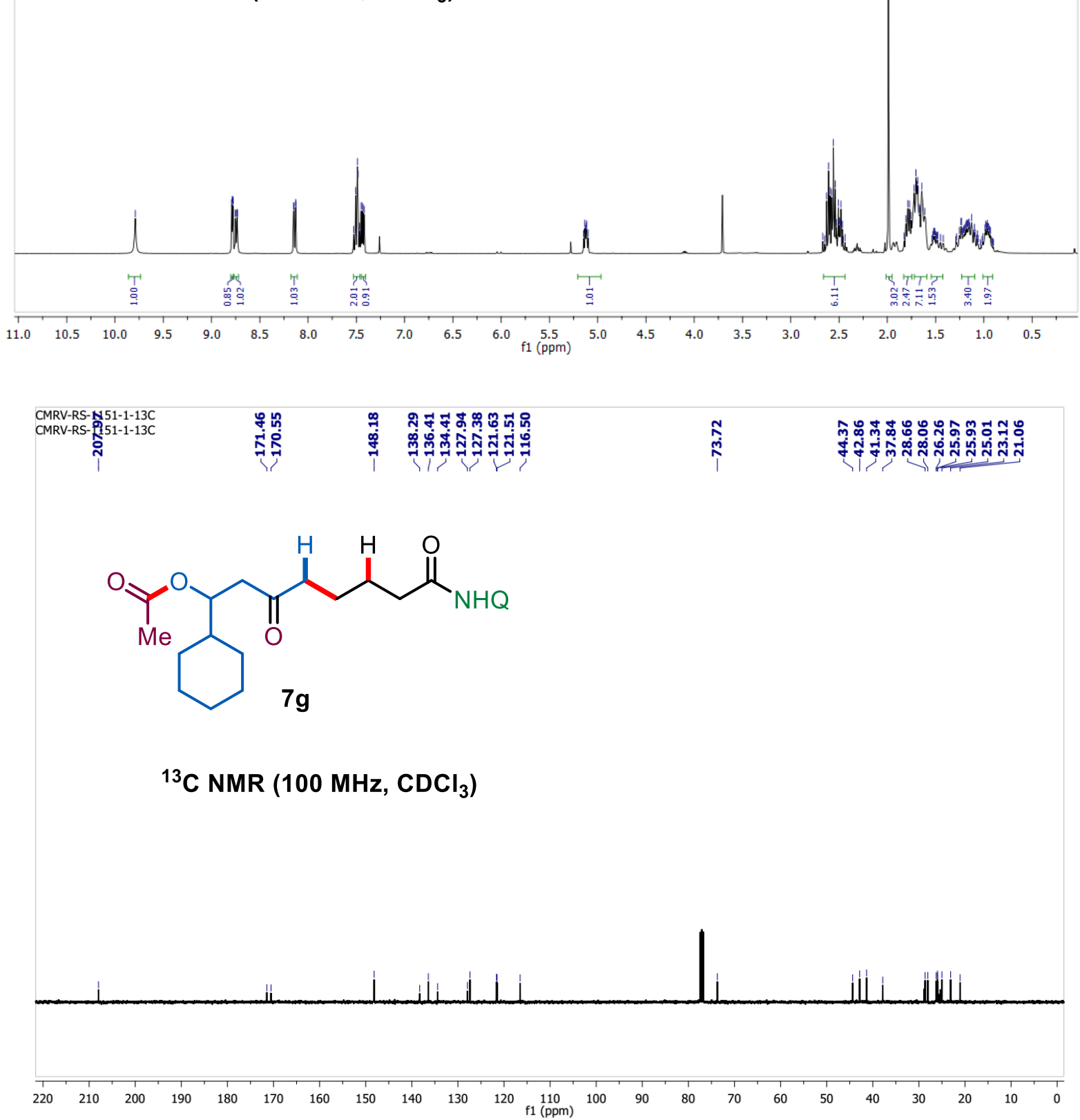
<smiles>CCCC(CC(=O)NO)C(=O)CC(C)OC(=O)C1CCC1</smiles>

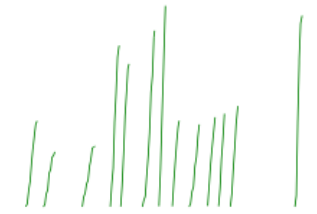

${ }^{1} \mathrm{H}$ NMR $\left(400 \mathrm{MHz}, \mathrm{CDCl}_{3}\right)$
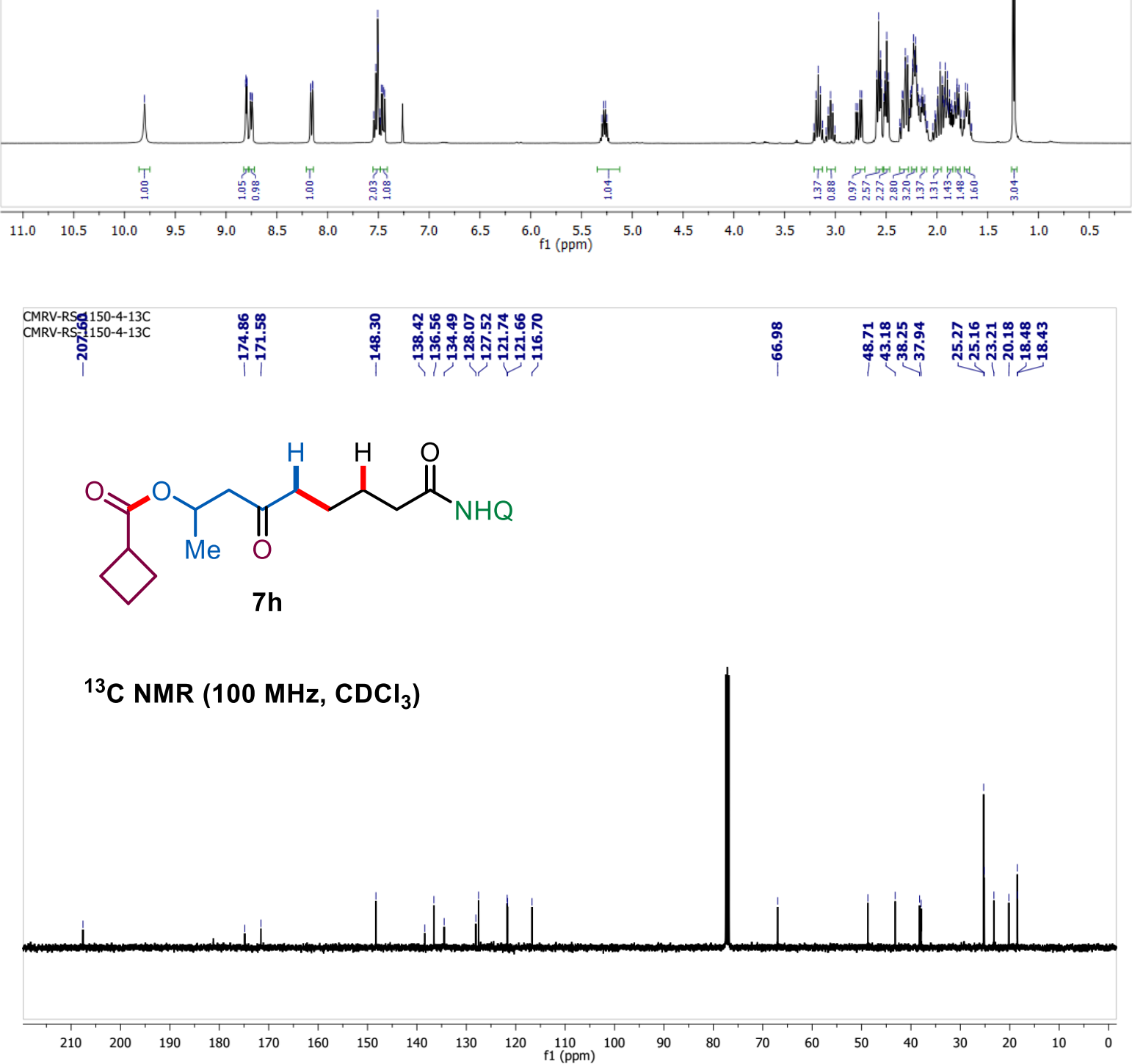

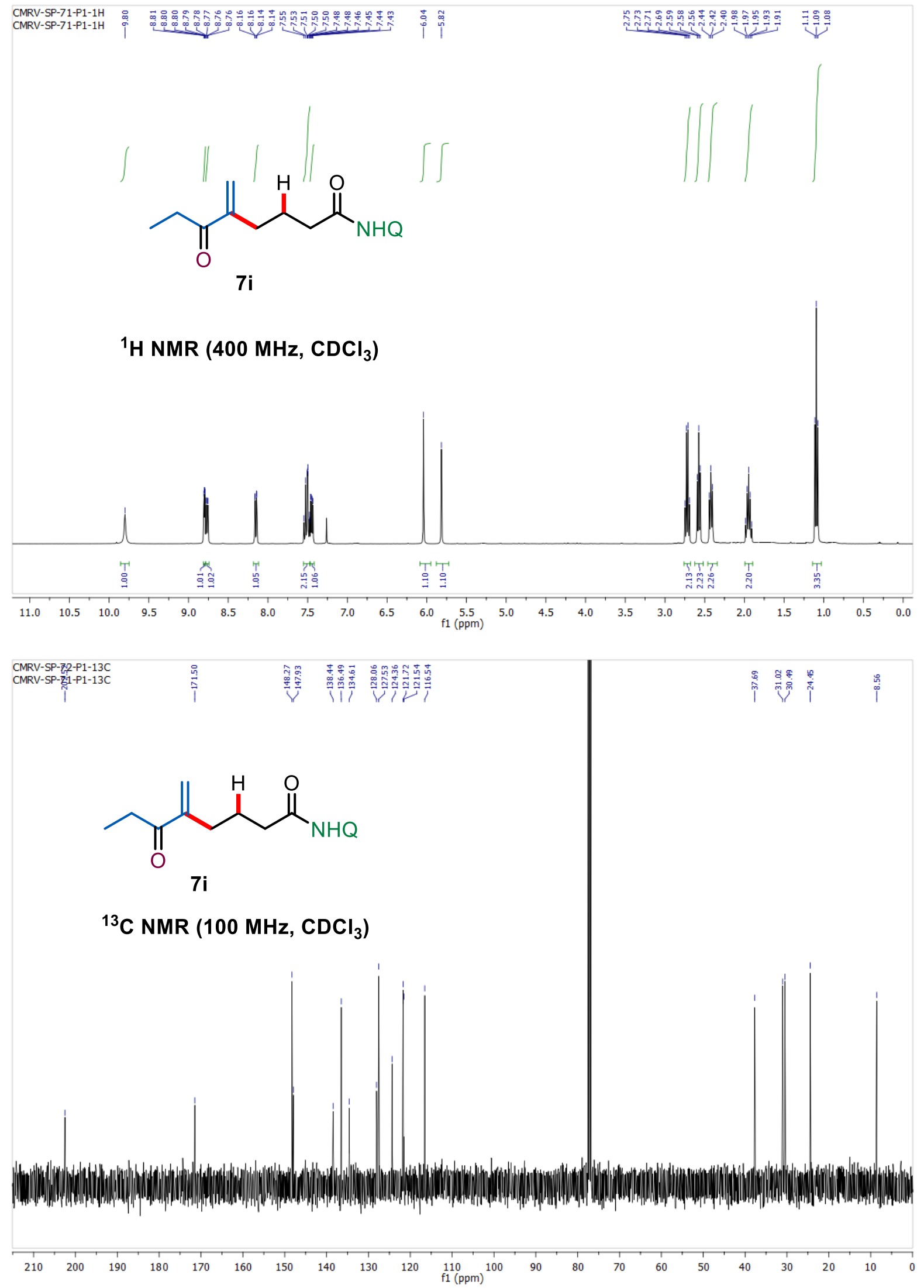


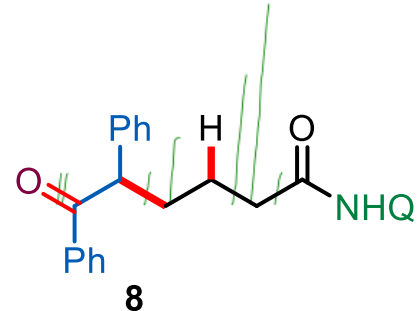

${ }^{1} \mathrm{H}$ NMR (400 MHz, $\mathrm{CDCl}_{3}$ )
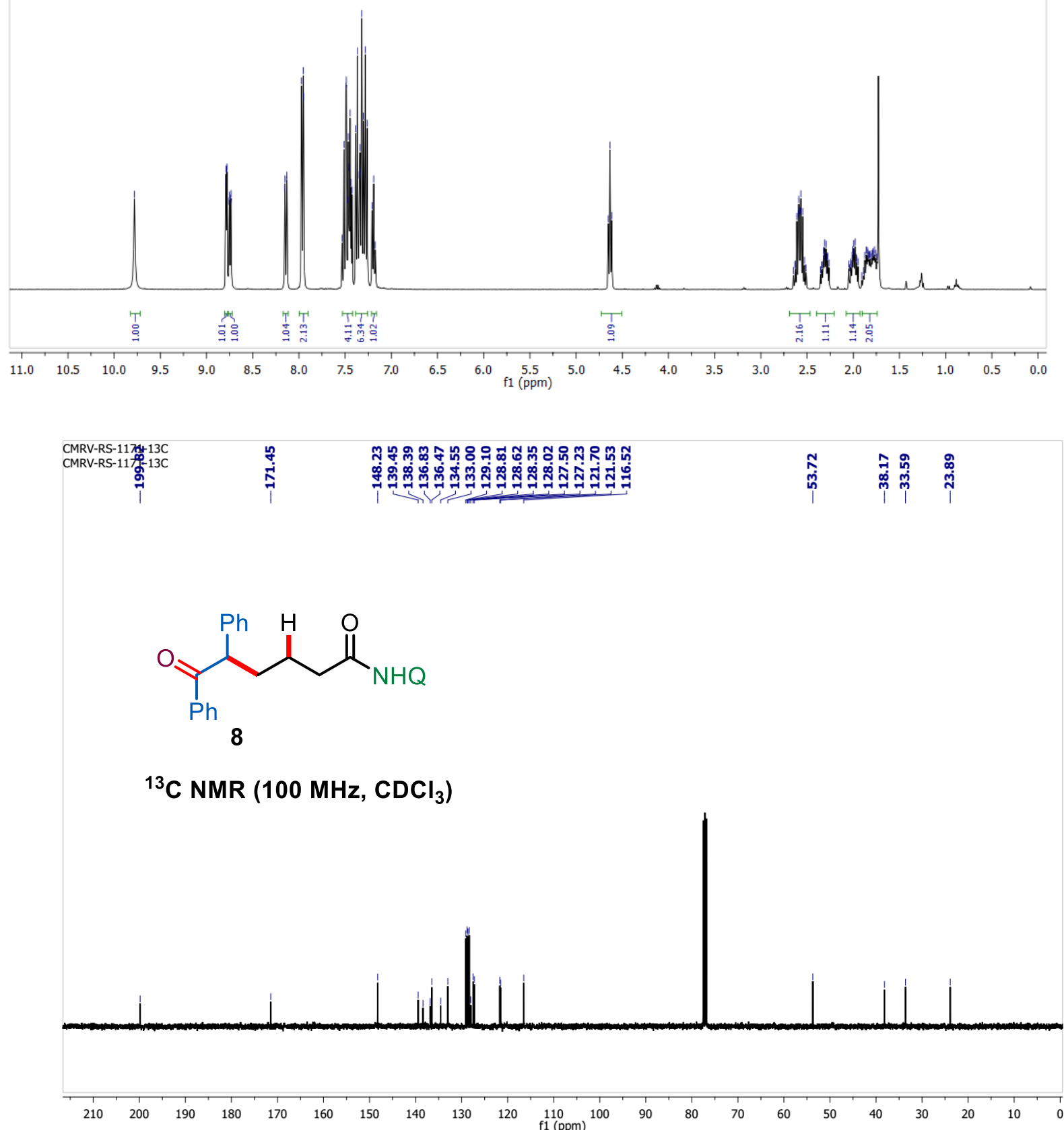

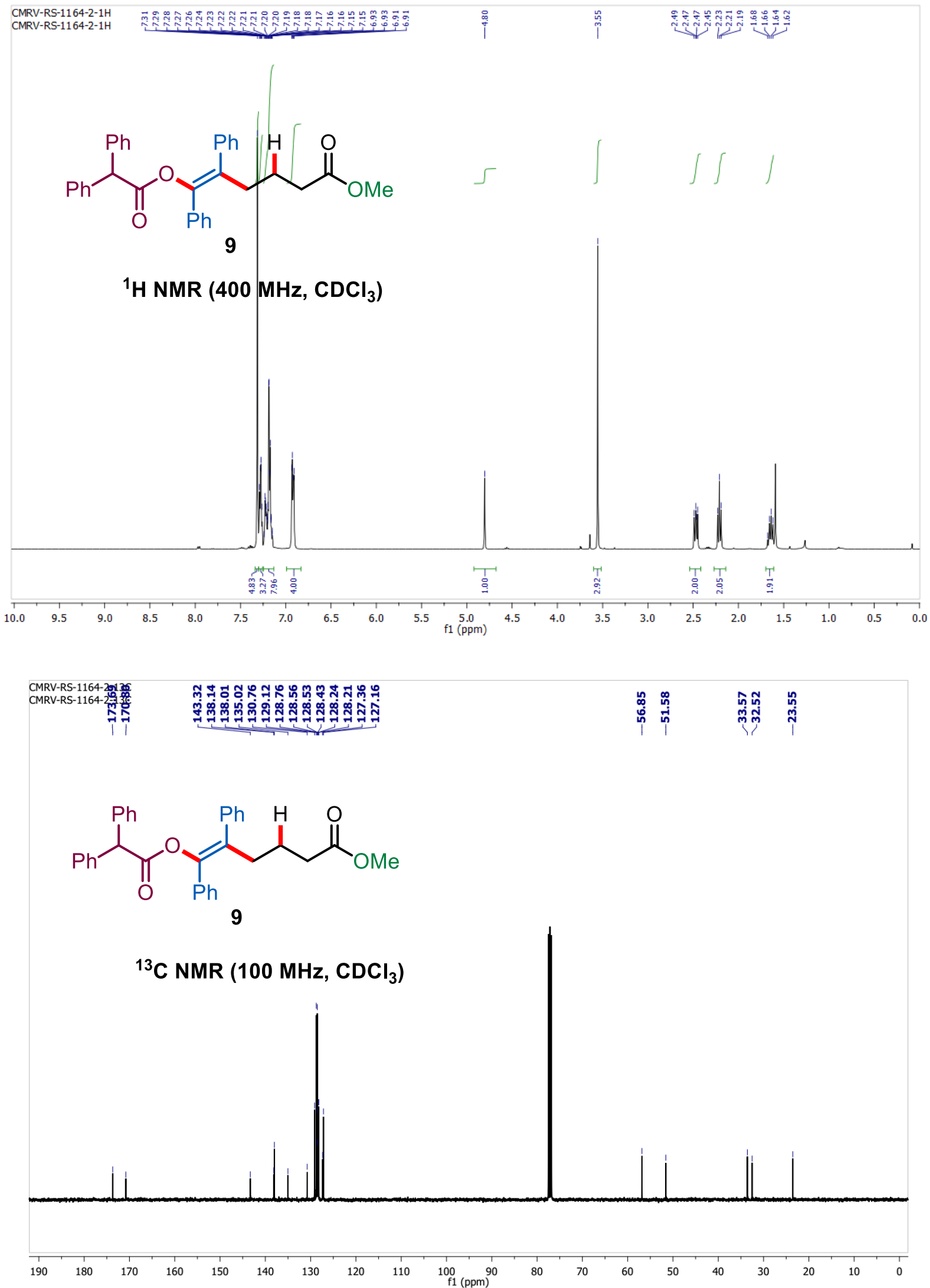